This is the final peer-reviewed accepted manuscript of:

L. Prodi, E. Rampazzo, F. Rastrelli, A. Speghini and N. Zaccheroni "Imaging agents based on lanthanide doped nanoparticles" 2015, Chem. Soc. Rev., 44, 4922-4952

SN - 0306-0012

DO - 10.1039/C4CS00394B

M3 - 10.1039/C4CS00394B

UR - http://dx.doi.org/10.1039/C4CS00394B

The final published version is available online at: https://pubs.rsc.org/en/content/articlelanding/2015/CS/C4CS00394B

Rights / License:

The terms and conditions for the reuse of this version of the manuscript are specified in the publishing policy. For all terms of use and more information see the publisher's website.

This item was downloaded from IRIS Università di Bologna (https://cris.unibo.it/)

When citing, please refer to the published version. 


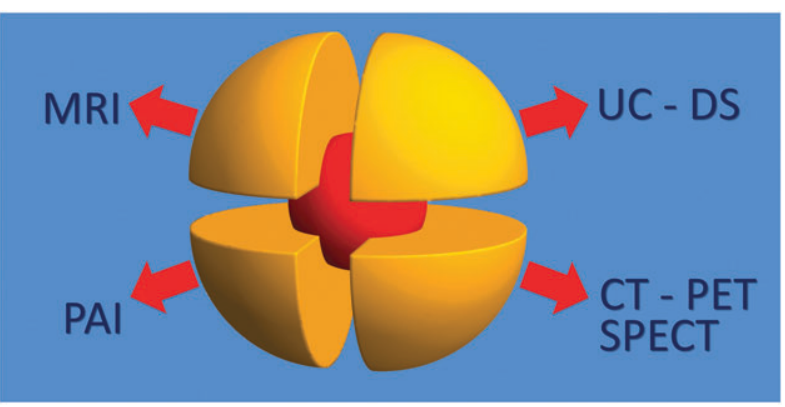

Imaging agents based on lanthanide doped nanoparticles

L. Prodi, * E. Rampazzo, F. Rastrelli, A. Speghini and N. Zaccheroni

This review summarizes the recent progress of single and multimodal imaging agents based on lanthanide doped nanoparticles.

Please check this proof carefully. Our staff will not read it in detail after you have returned it.

Translation errors between word-processor files and typesetting systems can occur so the whole proof needs to be read. Please pay particular attention to: tabulated material; equations; numerical data; figures and graphics; and references. If you have not already indicated the corresponding author(s) please mark their name(s) with an asterisk. Please e-mail a list of corrections or the PDF with electronic notes attached - do not change the text within the PDF file or send a revised manuscript. Corrections at this stage should be minor and not involve extensive changes. All corrections must be sent at the same time.

Please bear in mind that minor layout improvements, e.g. in line breaking, table widths and graphic placement, are routinely applied to the final version.

Please note that, in the typefaces we use, an italic vee looks like this: $v$, and a Greek nu looks like this: $\nu$.

We will publish articles on the web as soon as possible after receiving your corrections; no late corrections will be made.

Please return your final corrections, where possible within $\mathbf{4 8}$ hours of receipt, by e-mail to: chemsocrevarsc.org 


\section{Queries for the attention of the authors}

\section{Journal: Chem Soc Rev}

Paper: c4cs00394b

\section{Title: Imaging agents based on lanthanide doped nanoparticles}

Editor's queries are marked on your proof like this $Q 1, Q_{2}$, etc. and for your convenience line numbers are indicated like this $5,10,15, \ldots$

Please ensure that all queries are answered when returning your proof corrections so that publication of your article is not delayed.

\begin{tabular}{|c|c|c|}
\hline $\begin{array}{l}\text { Query } \\
\text { reference }\end{array}$ & Query & Remarks \\
\hline Q1 & $\begin{array}{l}\text { For your information: You can cite this article before you } \\
\text { receive notification of the page numbers by using the } \\
\text { following format: (authors), Chem. Soc. Rev., (year), DOI: } \\
10.1039 / \mathrm{c} 4 \mathrm{cs} 00394 \mathrm{~b} \text {. }\end{array}$ & \\
\hline Q2 & $\begin{array}{l}\text { Please carefully check the spelling of all author names. } \\
\text { This is important for the correct indexing and future } \\
\text { citation of your article. No late corrections can be made. }\end{array}$ & \\
\hline Q3 & $\begin{array}{l}\text { Do you wish to add an e-mail address for the } \\
\text { corresponding author? }\end{array}$ & \\
\hline Q4 & $\begin{array}{l}\text { "Accomulator" and "backgroung" appear to be spelled } \\
\text { incorrectly in Scheme } 1 \text {. Please could you supply a } \\
\text { corrected version (preferably as a TIF file at } 600 \text { dots per } \\
\text { inch) with your proof corrections. }\end{array}$ & \\
\hline Q5 & $\begin{array}{l}\text { The sentence beginning "Under a } 980 \mathrm{~nm} \text { excitation..." } \\
\text { has been altered for clarity, please check that the meaning } \\
\text { is correct. }\end{array}$ & \\
\hline Q6 & $\begin{array}{l}\text { "Shi" and "Bu" are not cited as authors of ref. 78. Please } \\
\text { indicate any changes that are required here. }\end{array}$ & \\
\hline Q7 & $\begin{array}{l}\text { Although there is a citation to Fig. } 33 \text { in the text, Fig. } 33 \\
\text { does not appear to have been provided. Do you wish to } \\
\text { provide this or would you like this citation to be removed? }\end{array}$ & \\
\hline Q8 & $\begin{array}{l}\text { A citation to Fig. } 18 \text { has been added here, please check } \\
\text { that the placement of this citation is suitable. If the } \\
\text { location is not suitable, please indicate where in the text } \\
\text { the citation should be inserted. }\end{array}$ & \\
\hline Q9 & Ref. 22: Please provide the page (or article) number(s). & \\
\hline Q10 & $\begin{array}{l}\text { Ref. 49: Can this reference be updated? Please supply } \\
\text { details to allow readers to access the reference (for } \\
\text { references where page numbers are not yet known, } \\
\text { please supply the DOI). }\end{array}$ & \\
\hline Q11 & Ref. 159: Please provide the initial(s) for the 4th author. & \\
\hline
\end{tabular}




\title{
Imaging agents based on lanthanide doped nanoparticles
}

\author{
L. Prodii, ${ }^{a}{ }^{a}$ E. Rampazzo, ${ }^{a}$ F. Rastrelli, ${ }^{b}$ A. Speghini ${ }^{\mathrm{c}}$ and N. Zaccheroni ${ }^{\mathrm{a}}$
}

Nanotechnology has recently allowed us to design and prepare nanoplatforms with the potential to face currently unresolved problems. Among these platforms, nanoparticles in particular are versatile objects that find applications in many different areas. In the vast ensemble of materials that have been explored to obtain nanoparticles with improved performances, we here focus our attention on lanthanide-based nanocrystals. These recently developed species are extremely interesting and well known particularly for their ability to emit anti-Stokes shifted light (upconversion) with relatively high brightness. Many advantageous characteristics of such materials are emerging, and their use as multimodal imaging agents is rapidly growing. We here survey some recent examples on this subject, mainly focusing on systems having NIR-to-NIR emission properties for in vivo applications.

While perceived as a frontier research field, nanotechnology is also an already well-established and fast growing discipline that

a Dipartimento di Chimica "G. Ciamician" and INSTM, UdR Bologna, Università di Bologna, Via Selmi 2, 40126 Bologna, Italy

${ }^{b}$ Dipartimento di Scienze Chimiche, Università degli Studi di Padova, Via Marzolo 1, 35131 Padova, Italy

${ }^{c}$ Dipartimento di Biotecnologie, Università degli Studi di Verona Ca' Vignal 1,

Strada Le Grazie 15, 37134 Verona, Italy finds widespread applications with health, environmental and social outcomes. It would be therefore redundant in this context to describe all the advantages of nanotechnology; yet, we would like to mention the key point that, in our opinion, explains the reason why this successful discipline has become now so fundamental: merging. Nanotechnology merges materials, properties and goals to obtain multimaterial-multifunctional-multitarget objects - an approach already paved by supramolecular chemistry - but pushed even further.

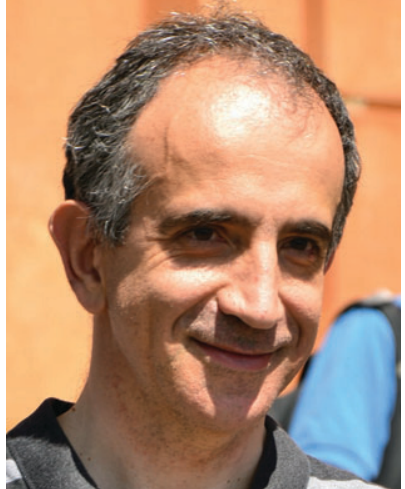

L. Prodi
Luca Prodi received his $P h D$ in 1992. In 2006 he become a full Professor of General and Inorganic Chemistry at the University of Bologna, and in May 2015 he was elected Head of the "Giacomo Ciamician" Department of Chemistry. His research activity is focussed on the synthesis and characterization of luminescent labels and sensors, using the supramolecular approach for the analyte recognition and the signal transduction steps. In this context, he is also actively working on the synthesis and characterization of metal and silica nanoparticles, in order to obtain more efficient sensors and labels through signal amplification, mainly for bio-medical applications. He is an inventor of 4 international patents and a cofounder of two spin-off companies.

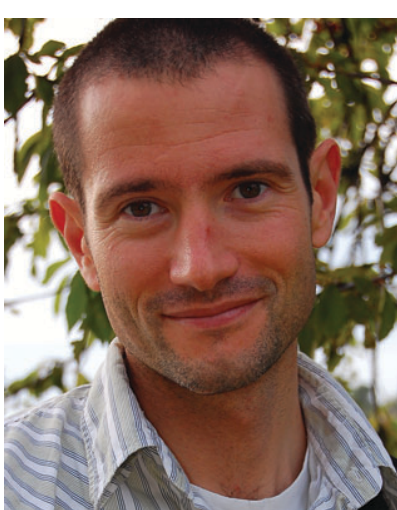

E. Rampazzo
Enrico Rampazzo was born in Verona in 1973. He completed his $\mathrm{PhD}$ at the University of Padua under the supervision of Umberto Tonellato and Fabrizio Mancin. He was a FIRB young researcher and a postdoctoral fellow with Luca Prodi and Marco Montalti at the Photochemical Nanosciences Laboratory of the University of Bologna (Italy). He is now an assistant professor in the same group. His research focuses on the design and synthesis of luminescent

and (electro)luminescent systems based on dye doped silica nanoparticles for the development of labels and sensors. 
This merging ultimately leads to nanoplatforms that may become very powerful tools to face current unresolved problems. Environment and energy are for sure two of the greatest issues that the modern world must address nowadays. Indeed, much effort has been directed towards the healing of the human body through medicine, in the form of prevention, diagnosis, and therapy. This last point is so important that the word theranostics ${ }^{1}$ has been introduced to indicate a treatment strategy that combines therapeutics with diagnostics. ${ }^{2}$

10 Clearly, in the design of platforms to be used in vivo, there exist many and urgent constrains such as toxicity, ${ }^{3}$ stability, environmental compatibility, time of clearance, and output signal reliability and intensity. ${ }^{4}$

Many are the possible techniques in modern medical ima-

15 ging that can be exploited to generate a signal to be tracked. Non-invasive imaging methods using exogenous chemical

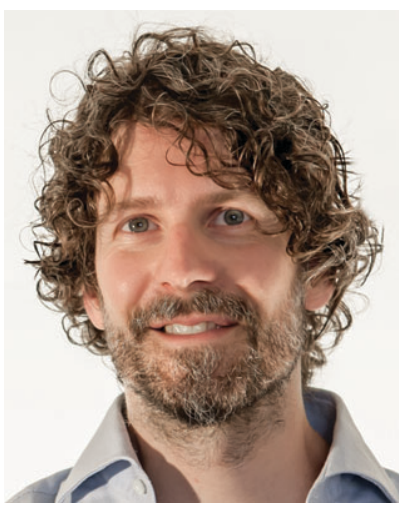

F. Rastrelli
Federico Rastrelli was born in 1973. He completed his PhD under the supervision of Alessandro Bagno and Gianfranco Scorrano at the University of Padova, where he currently holds an assistant professor position. In the group of Alessandro Bagno he has been working on experimental $N M R$ and quantum chemical calculations in small diamagnetic and paramagnetic molecules. His present research interests include the design of NMR methodologies for the investigation of nanosystems and the study of transport 35 phenomena in complex matrices.

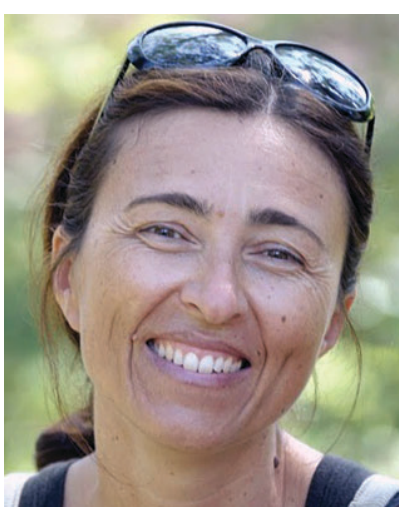

N. Zaccheroni Autonoma Metropolitana (Mexico), Humboldt-Universität zu Berlin (Germany) and a visiting professor at the Institut National de la Recherche Scientifique (Canada). His research interests are focused on the development of nanomaterials and nanostructures in colloidal forms for biomedical diagnostic and photonic applications. contrast agents (CA), in fact, are a booming frontier research area with an expected breakthrough in diagnostics and in all medical activities that can be imaging-guided such as surgery, drug delivery and therapy monitoring. ${ }^{5}$ The main challenges for in vivo imaging, to date, include the development of new contrast agents with increased sensitivity and specificity. ${ }^{6}$ The most promising approach that has emerged from the research efforts worldwide points distinctly toward nanostructured and nanosized probes ${ }^{7-10}$ that are compatible with most imaging modalities and that can be optimized for targeting, ${ }^{11}$ multimodality and activation. The various imaging techniques differ significantly in fundamental features (Table 1) such as the resolution, penetration depth, time of analysis and cost - with pros and cons depending on the investigation to be addressed. It is straightforward to understand how the combination of two or more of them - bringing to multimodality - can help overcome the single constraints. ${ }^{12}$ Even if this approach is already followed in medical applications, pushing forward multimodal imaging still remains one of the main goals of in vivo imaging research. Magnetic resonance imaging ${ }^{13}$ (MRI), computed X-ray tomography $^{14}$ (CT), nuclear medical imaging ${ }^{15}$ (NM) such as positron emission tomography (PET) and single-photon emission computed tomography (SPECT), ultrasound imaging ${ }^{16}$ (US) are the modalities that already find a large clinical application. It is worth pointing out that MRI and PET instrumentation suffer from high costs, something which plays a crucial role in modern healthcare systems. In addition MRI has a quite low sensitivity (together with CT) and very long acquisition times in comparison with other tomographic techniques (around 30 minutes versus seconds-minutes). Conversely, MRI can offer a non-limited penetration depth, a high resolution and a high soft-tissue contrast.

All these advantages, along with the virtual absence of health risks, make this technique one of the most commonly

Nelsi Zaccheroni studied and obtained her PhD in Chemical Sciences (1997) from the University of Bologna (Italy), where she is now an Associate Professor in Chemistry since September 2014. She spent one year (1997-1998) as a postdoctoral fellow within a TMR-CEE project at the University College of Dublin (Ireland) where she started to approach the study of nanomaterials. Her research interests are now mainly focused

on luminescent systems for imaging and sensing, both molecular and nanostructured in particular based on silica, metal and core-shell multi-material nanoparticles.

5

10

5 
Review Article

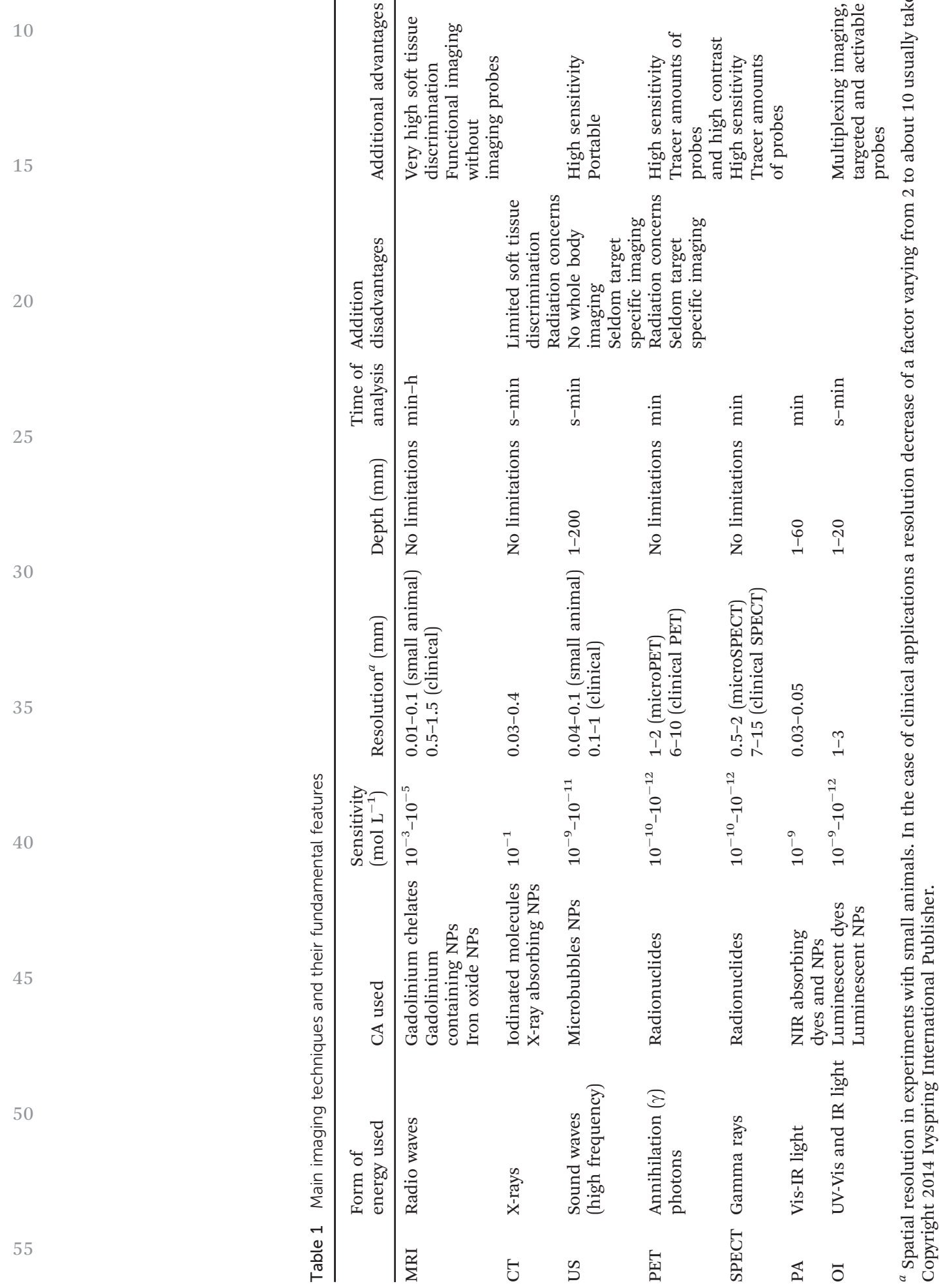


1 used in the world together with ultrasound imaging (US, i.e. sonography) - that is also investigated for therapeutic treatments. ${ }^{17}$ The latter technique benefits from a high sensitivity and a short time of measurement, suffers from a limited penetration (1-200 mm). The same drawback also affects CT together with relatively poor soft tissue contrast and the concern about the X-ray radiation. Safety is an issue also in the case of NM (Nuclear Medicine) that, despite the limited spatial resolution, can ensure no limits in penetration and the highest

10 sensitivity. In this already rich but complex scenario, significant improvements (implying very strong expectations) are estimated from two imaging modalities, optical imaging ${ }^{10}$ (OI) and photo-acoustic imaging ${ }^{18}$ (PAI), which to date have still very limited clinical translation.

These techniques are attracting funding and great research efforts $^{19,20}$ that are estimated to bring about important achievements in a relatively short time. OI and PA share very high sensitivities and short time of measurements. OI can suffer from a relatively limited resolution in comparison with other imaging modalities but, on the other side, it has a quite unique versatility, such as the possibility to exploit light not only for diagnostic purposes but also for therapeutic treatments (photodynamic $^{21}$ and photothermal therapy, PDT and PTT respectively $\left.{ }^{22-26}\right)$. Moreover, properly designed probes can allow multiplexed imaging ${ }^{27,28}$ a very valuable high-throughput possibility that, monitoring many parameters/analytes at the same time, can drastically lower the required sample sizes and/or the time of analysis. The main drawback of OI and PAI, however, is their low investigation depth $(<20 \mathrm{~mm}$ for OI and of $60 \mathrm{~mm}$ for 30 PAI) in the case of in vivo applications. Due to the absorption and scattering of tissues (especially between 400 and $700 \mathrm{~nm}$ ), in fact, the propagation of the excitation and, for OI, emission radiation is an issue. This may be at least partially overcome in the future thanks to the anticipated great advances of real/time endoscopic $^{29}$ and laparoscopic imaging introduced by the new generations of more and more miniaturized instruments. In addition, OI is finding growing interest from surgeons since it can help guiding their procedures by visualizing, for example, a tumour mass to be resected with great accuracy.

40 A necessary step to improve the penetration depth is anyway to optimize the probes to exploit the biological optical transparency window (650-1800 nm), a region that takes into account absorption and scattering caused by the species and structures of the different skin layers. ${ }^{30}$ This region can be divided in the

45 so-called NIR-1 (650-950 nm, where the tissue scatter and autofluorescence are still quite high), NIR-2 (1000-1350 nm, where the water absorption increases) and NIR-3 (1500-1800 nm). ${ }^{20}$ The reason for the two gaps that separate the three NIR (near infrared) ranges can be found in two absorption peaks of water, the first one centred at around $980 \mathrm{~nm}$ and the second much higher one at about $1450 \mathrm{~nm}$ (Fig. 1). The excitation or emission light falling in these two sections is therefore partially absorbed and thus suffering of limited penetration. It is also worth pointing out another detrimental consequence: the excitation via laser light at $980 \mathrm{~nm}$, very common in the structures involving $\mathrm{Yb}^{3+}$ ions, causes the transformation of

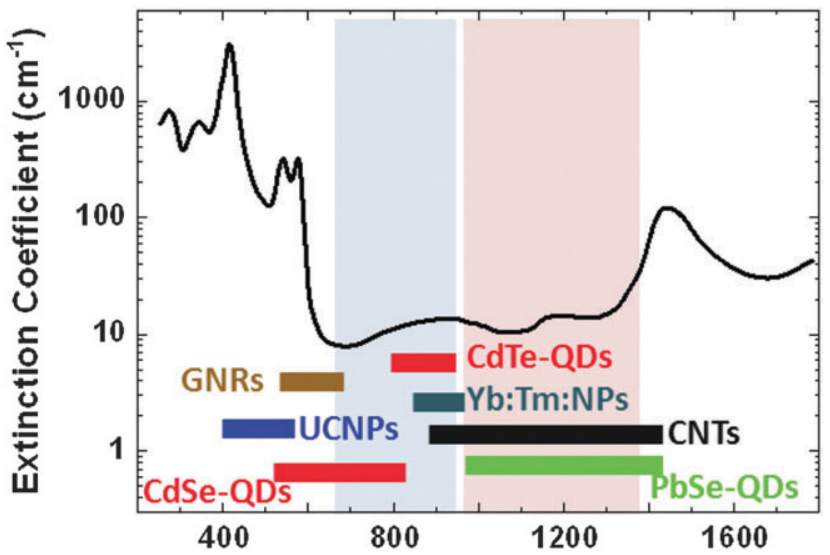

1

Fig. 1 Attenuation coefficient of human blood (oxygenated) in the 300$1700 \mathrm{~nm}$ spectral range. NIR-1 (blue color) and NIR-2 (pink color) biological windows are indicated. The emission spectral ranges of luminescent NPs classes used for fluorescent bioimaging are included (GNRs, gold nanorods; UCNPs, upconverting NPs; QDs, quantum dots; CNTs, carbon nanotubes). Adapted from ref. 31 Copyright 2014 Wiley-VCH Verlag GmbH \& Co. KGaA, Weinheim.

the absorbed energy into a local heating of biological samples, a potentially very critical problem. All this has to be carefully taken into account in designing suitable systems for applications in biomedical imaging. Such systems must combine NIR absorption and emission of light, to ensure a significant increase in penetration ${ }^{20,23}$ with other advantages such as low damage of tissues and cells, which is always a concern with long-term UV excitation, and reduced light scattering and auto-fluorescence.

A great number of possible variables govern the properties of lanthanide doped NPs: this complexity makes such materials very challenging but extremely versatile at the same time. Several examples of NPs have been developed, which are chemically and photochemically stable, with sharp emission bands (including intense anti-Stokes ones), NIR excitationemission, long lifetimes and low toxicity. Their properties, however, are not only confined to luminescence - in many permutations they are also suitable candidates for imaging techniques such as MRI (see Section 4), CT and also NM (see Section 5). In the last few years the area of lanthanide doped NPs has therefore boost up with the application of single and multimodal systems to imaging ${ }^{32}$ and therapy, ${ }^{33,34}$ but much effort is still needed to translate them into the clinic.

The multifaceted interest in these materials is certainly testified by the impressive number of publications and reviews that are appearing in the literature daily describing the production of lanthanide-doped nanocrystals ${ }^{20,33,35-38}$ and their possible applications. ${ }^{19,20,33,39-42}$ Several factors such as the morphology, crystalline phase, size, and components of these nanomaterials are crucial parameters acting on their electrical, photophysical, magnetic, and colloidal stability properties. ${ }^{43,44}$ Most of the synthetic routes - whose implementations are unfortunately still often linked to a trial-and-error approach focus on achieving a rational control over these elements.

In this constantly evolving scenario, we have decided to give the reader an overview of the state of the art, not even trying to

.


1 be exhaustive but rather focusing on lanthanide-doped nanocrystals both absorbing and emitting in the NIR (1 or 2) region. We have chosen this restricted subject as the most promising and innovative in the development of theranostic nanomaterials for the future of mankind. In this context, NIR contrast agents, specifically targeted and eventually activated ${ }^{40,45}$ for drug delivery, represent some of the most relevant results to be achieved in the next future. In this review article we have classified the results found in the literature depending on the imaging modality for which the lanthanide doped nanoparticles were designed. For each of them we begin with a brief introduction on the principles at the basis of the method. The theoretical outline introducing the lanthanide based nanocrystals as MRI contrast agents (Section 4) has been expanded in 5 proportion to the complexity of the subject: we have tried, however, to keep the dissertation within reach of a wide readership.

Finally, since lanthanide doped nanocrystals are appealing materials that will take to unpredictable and bright advancements in many disciplines of high social impact, we have tried to make the new achievements as accessible as possible also to interested researchers working in different fields.

\section{2. Lanthanide-doped nanocrystals for optical imaging}

Among the variety of possible luminescent materials only a very limited group presents NIR adsorption and emission properties. Materials based on lanthanide ions $\left(\mathrm{Ln}^{3+}\right.$, from lanthanum to lutetium) are included in this set. Lanthanide ions are characterized by the progressive filling of the highly shielded 4f orbitals, originating many states with energies determined by the combinations of three contributions: the inter-electronic repulsion, the spin-orbit coupling and the ligand field. The lower energy excited states in the lanthanide ions are always determined by the rearrangement of the electrons in the $f$ orbitals that give rise to very narrow $\mathrm{f}-\mathrm{f}$ absorption bands (with semi-width even lower than $1 \mathrm{~nm}$ ) and with very low absorption 0 coefficients (rarely above the unity) due to their forbidden character. These transitions give rise to the emission of these ions that are again very narrow and, due to their low probability, with very long radiative lifetimes - often in the range of milliseconds. Frequently, the nonradiative deactivation 5 through the vibronic coupling with species in close proximity or via excited states with different configurations is very efficient. Therefore, to obtain highly luminescent materials based on $\mathrm{Ln}^{3+}$ ions, there are two problems to be overcome: (i) the inefficient process of their direct excitation and (ii) an efficient nonradiative deactivation. The research of possible solutions constitutes a very lively research field since several decades and many different ways have been proposed to obtain luminescence from lanthanide ions through indirect sensitization processes from light-absorbing systems. Lanthanide coordination can allow efficient energy transfer from light harvesting organic-antenna chromophores, ${ }^{46}$ or by organic aromatic ligands that are directly bound to the lanthanide centres or, again, by light harvesting metal-organic antenna chromophores in heteronuclear metallo-organic complexes. ${ }^{47}$ There are also other less traditional and very intriguing possibilities such as doping lanthanide inorganic matrixes with other metal ions to obtain luminescent glasses or more organized nanosized materials such as lanthanide doped nanoparticles.

Lanthanide doped nanoparticles, which are the topic of this review, are gaining more and more attention in the last few decades merging the advantages of nanomaterials with unique optical properties. ${ }^{33}$ They are formed by a suitable dielectric host lattice where trivalent lanthanide ions are dispersed at low concentration. Their emission properties are influenced by the nature, the composition and the structure of the nanoparticle, but they are not directly dependent on their size or shape as it is typical of many other nanostructured systems such as quantum dots (QDs).

These luminescent properties differ significantly by other luminescent nanoparticles for the possibility of giving rise to efficient anti-Stokes emission processes. The classic downshifting emission (DS), which is advantageously used also with lanthanide-doped nanocrystals, consists in the conversion of the higher energy excitation photons into lower energy ones following the Stokes law. In contrast, in up-conversion emission (UP) processes the sequential absorption of two or more photons and energy transfer processes allow the emission of higher energy photons. ${ }^{48}$ Interestingly, as we will see, some researchers have also conceived lanthanide-doped nanocrystals in which both modalities can be used.

Whichever the mechanism (DS or UC), since the excited state responsible for the emission is for almost all these systems centred on the lanthanide ion, they share common features. In particular, together with the already mentioned sharp emissions and rather long excited state lifetimes, the luminescence intensity depends on the nature and concentration of the components, on the nature of the matrix and on the synthetic conditions.

The nano-range size is of central importance for biomedical applications, since the dimension entails internalization and release in cells, organs and biological hosts. Other fundamental aspects are solubility, ${ }^{19}$ biocompatibility ${ }^{49}$ and low-toxicity ${ }^{50}$ that depend very much upon the nature of the surface of lanthanide-doped nanoparticles, which can be conveniently functionalized, ${ }^{39}$ as already mentioned, to allow also bioconjugation.

\subsection{UCNPs: anti-Stokes-emitting NPs}

The most intriguing emission property of lanthanide doped nanophosphors is the UC luminescence. Ion-doped upconverting bulk materials are known from more than 50 years, ${ }^{51}$ and the understanding of the role of the different mechanisms ${ }^{52}$ involved in this phenomenon ${ }^{52,53}$ was really improved by the availability in the following years of powerful NIR laser exciting sources. These upconverting bulk materials have been initially mainly used in the area of optical devices (such as in solid state lasers $)^{54}$ and only the advent of upconverting nanoparticles (UCNPs) has opened up the way to bio-medical applications, ${ }^{55,56}$ 

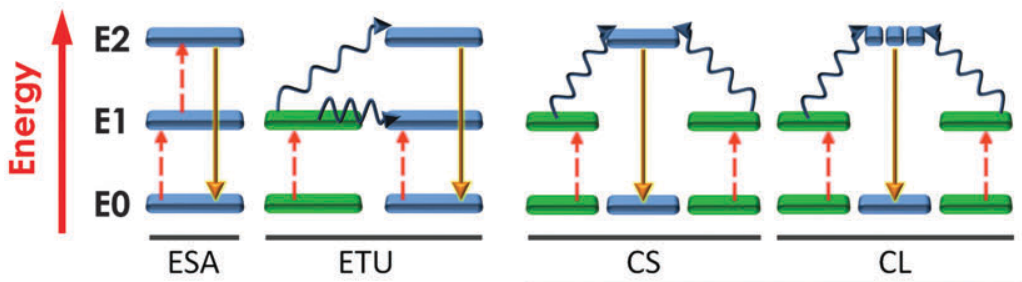

CU Legend: . $\begin{array}{ll}\text { E0-Ground State } & \\ \text { E1 - Excited State } 1 \quad \text { Excitation } & \text { Proces }\end{array}$

Scheme 1

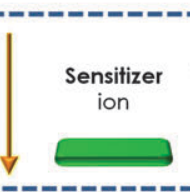

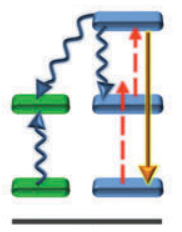

PA

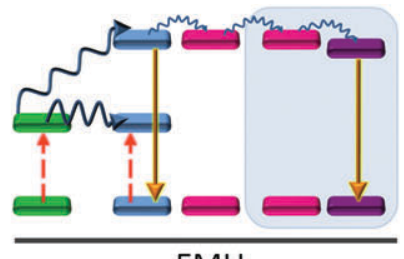

EMU Activator (or Accomulator
in EMU) in $\mathrm{EMU}$
ion ion

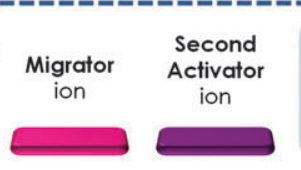

Energy level schematization of the main reported lanthanide ion anti-Stokes mechanisms (see the text for the acronysms).

also thanks to the recently improved emission efficiency that has always been an issue in upconversion materials. This can be attributed to the careful design of materials with customized and optimized optical properties. ${ }^{40,41,57}$ The possible strategies are many and the characteristics of UCNPs can be tuned via the proper choice of the right host-dopant combination, via the engineering of the nanostructure (for example with an appropriate core-shell design $)^{58}$ or via the addition of suitable further species able to exchange energy with the other constituents. We can say that, generally, all the strategies involve the combination of three main components: a host matrix, a sensitizer and an activator (the emitting $\mathrm{Ln}^{3+}$ ion).

Host materials (matrix) play a fundamental role in the UC 30 being not simply scaffolds, they have to present necessary features such as: (i) the right lattice dimensions to tightly hold the $\mathrm{Ln}^{3+}$ guest ions, (ii) a low lattice phonon energy to minimize nonradiative processes, (iii) a negligible absorption in the spectral working region and (iv) a high chemical and photophysical stability. Alkaline earth fluorides are, to date, the best performing ones and therefore the most studied also in the area of biomedical imaging, although oxide based ones (e.g. sesquioxides or oxosulfides) can be a valid alternative. ${ }^{59-61}$

The $\mathrm{Ln}^{3+}$ guest ions providing the UC emission are called activators and can present bands in a large spectral range under very different excitation power densities (ions with ladder-like disposition of the electronic states require low power, the others much higher one). To avoid cross-relaxation energy losses, it is necessary to use very low doping degrees of activators $(<2 \%)$ and this is detrimental for the brightness: to overcome this problem the most common approach is to prepare doubly-doped nanoparticles that present a sensitizer with a much higher doping concentration (around 20\%) together with the activator. Its function is to absorb energy in the NIR region (and therefore a large cross-section is needed) and to efficiently transfer it to the emitting ions. $\mathrm{Yb}^{3+}$ is the candidate that better matches these requirements and by far the most common sensitizer.

UC luminescence takes place via a number of different mechanisms, all favoured by the long lifetime of $\mathrm{f}-\mathrm{f}$ transitions that allows energy transfer among lanthanide ions and increases the probability of sequential excitation of the same ion. These mechanisms could be distinguished in five different processes (Scheme 1): (i) excited-state absorption (ESA), (ii) energy-transfer upconversion (ETU), (iii) cooperative upconversion (CU) (where one can discriminate cooperative sensitization upconversion (CS) and cooperative luminescence upconversion ${ }^{62}$ (CL)), (iv) photon avalanche (PA) and (v) energy migration-mediated upconversion (EMU). ${ }^{63}$

We would like to present here very shortly the differences of these various mechanisms trying to recall only the essential information that could be of help for the reader, without any ambition to be exhaustive on the topic that has already been brilliantly treated in detail by many researchers. ${ }^{33,52,64-66}$

(i) ESA takes place in ions presenting a ladder-like disposition of the electronic states $\left(\mathrm{Er}^{3+}, \mathrm{Ho}^{3+}, \mathrm{Tm}^{3+}\right.$ and $\left.\mathrm{Nd}^{3+}\right)$ with almost identical energy separation among successive ones. This, together with their long lifetime, allows the sequential absorption of two photons by the same activator ion that then emits from the second energy level.

(ii) ETU needs a neighbouring sensitizer and an activator to occur and it is therefore dependent on dopant concentrations. The sensitizer absorbs photons and via sequential energy transfer processes (one of them occurring between two ions both in an excited state ${ }^{52}$ ) takes the activator to its second emitting level generating UC emission. This process is the one at the bases of the high efficiency of the $\mathrm{Yb}^{3+}$ doped UCNPs that are to date the most exploited and common ones.

(iii) CU can be distinguished in cooperative sensitization or cooperative luminescence upconversion. CS involves three ions, generally two sensitizers and one activator, and the simultaneous energy transfer of the photons absorbed by the sensitizers to the emitting ion populates its upper level that deactivates via UC emission. In the CL, in contrast, the simultaneous deactivation of two excited ions provides an emission with twice the energy of the single excited states. CL presents a much lower efficiency than the other mechanisms and therefore it is not usually exploited in the engineering of UCNPs.

(iv) $\mathrm{PA}^{53}$ requires laser-pump radiation above a critical excitation power and withenergy a bit higher than the one of the first excited state of the emitting ion. Under these 
1 conditions an initial ESA occurs that induces both a very weak UC emission from that activator and an efficient crossrelaxation that takes the neighboring sensitizer to its excited state, from where it is then able to transfer its energy back to the activator. This constitutes a kind of avalanche effect in the population of the first excited state of the activator. Long rise time to build up (seconds) and the existence of a critical pump threshold make easy to discriminate the occurrence of the PA mechanism.

10 (v) EMU is the most recently proposed mechanism, it starts with an ETU step but it involves four different types of interacting centers (four lanthanide ions) with proper and defined concentrations and arranged in a multilayered (core-shell) structure. ${ }^{67}$ In the first step of the EMU process the sensitizer

15 (I) absorbs and then transfers its energy to the higher excited state of the accumulator (II), after successive energy transfers to a first migrator (III) and then through the layers interface to other migrators the energy is finally populating the excited state of the activator (IV) ion to give the UC luminescence.

It is important to highlight that the UC efficiencies of the various mechanisms are very different. In particular ETU is the most efficient one and together with EMU (that can be seen as a more complex ETU case) are by far the most exploited in the design of UCNPs. In contrast, $\mathrm{CU}$ and above all PA require a high doping concentration and therefore they are much more common in bulk materials. Indicative orders of magnitude for the various UC efficiencies were already reported by F. Auzel, ${ }^{52}$ his studies have been the foundations of this field and proposed this general trend: ETU $>$ ESA $>\mathrm{CU}(\mathrm{CS}>\mathrm{CL})>$

30 PA. The most important point, however, is that the complexity of the UC emission processes makes always challenging to carefully characterize the optical properties of UCNPs. In particular the determination of their emission quantum yield (QY), defined as the ratio of the number of the emitted UC photons to the number of absorbed photons of the excitation radiation, ${ }^{33}$ is not at all trivial with the direct consequence of a difficult comparison of the performance of different particles. This is very much due to the power dependence of the UC intensity, which is not even univocal since UCNPs are subject to different saturation levels: for the same host, activator and doping degree, it depends on the size of the nanoparticle. The importance to have protocols allowing a consistent evaluation of the QY to compare the results coming from different experiments, studies and applications has already been dis5 cussed. ${ }^{42,68}$ A comprehensive exposition of the methods to calculate the QY in UCNPs is beyond the aims of this review, we would only like to stress that the QY of the UC emission is usually lower than $1 \%$. This point is of fundamental importance since diagnostic imaging and theragnostic applications require appropriate brightness that, in the case of UCNPs, can even decrease with a reduction of the size. This is due not only to the lower number of activators in a smaller volume (at the same doping degree) but also to quenching processes entailing the surface. In the nanoparticles, in fact, the surface to volume ratio is very high exposing a relevant percentage of luminescent centers to deactivation involving the solvent, other species in the environment, or even surface lattice defects that can act as long distance quenchers for inner activators via energy migration. The rational design of the external modification of UCNPs is thus fundamental not only to control their solubility and possible conjugation properties but also to minimize these possible detrimental effects and increase the overall light emission efficiency. ${ }^{67}$ To the same goal a proper design of the UCNPs can allow the control of their excitation dynamics again of great importance in the pursuing of brighter and brighter particles. ${ }^{65,66}$

The controlled planning of these systems is not at all trivial and many research groups spent significant efforts to optimize UCNPs for optical imaging applications starting from the doping with non $\mathrm{Ln}^{3+}$ ions or from the design of the matrix. $\mathrm{Gu}$, Zhao and co-workers ${ }^{69}$ demonstrate that upon changing the concentration of dopant $\mathrm{Li}^{+}$in $\mathrm{GdF}_{3}: \mathrm{Yb}, \operatorname{Er}(20,2 \mathrm{~mol} \%) \mathrm{NPs}$ it is possible to modulate the energy back-transfer process induced by the alkaline metal ions. Under a $980 \mathrm{~nm}$ excitation the ratio of the green and red emission of $\mathrm{Er}^{3+}$ decreases with an increase in $\mathrm{Li}^{+}$doping resulting in a yellow to an enhanced bright red upconversion emission suitable for NIR-to-NIR biological imaging. On the other side, also a matrix change, with a consequent different crystallinity, can induce a 10-fold enhancement of the UC luminescence in sub-20 nm cubic $\mathrm{NaLuF}_{4}: 20 \% \mathrm{Yb}, 1 \% \mathrm{Tm}$ nanocrystals as described by Yang, Li and co-workers. ${ }^{70}$ This bright agents presenting both excitation (980 $\mathrm{nm})$ and emission (800 $\mathrm{nm}$ ) in the NIR region were used for in vivo imaging in animals and could reach a penetration depth of $1.5 \mathrm{~cm}$ in pork tissue. This last feature is a crucial point for in vivo optical imaging and, due to the transparency biological window discussed in the introduction, it greatly benefits by NIR-to-NIR luminescent probes. For this reason most of the literature on lanthanide based UCNPs for biomedical applications focuses on ytterbium (sensitizer) doped NPs that can be conveniently excited at $980 \mathrm{~nm}$ with common continuous wave (CW) lasers of various power density. The co-doping with one or more suitable activator ions yields NIR luminescence ensuring also the efficient collection of the emitted light in depth. The most common couple combinations are $\mathrm{Yb}^{3+} / \mathrm{Er}^{3+}, \mathrm{Yb}^{3+} / \mathrm{Tm}^{3+}$ and $\mathrm{Yb}^{3+} / \mathrm{Ho}^{3+}$, with an UC emission in the NIR-1 spectroscopic range. In particular, many $\mathrm{Tm}^{3+}$ doped nanocrystalline fluoride hosts have been investigated, such as $\mathrm{NaYF}_{4},{ }^{71,72} \mathrm{NaGdF}_{4},{ }^{73} \mathrm{LiYF}_{4}{ }^{74}$ and $\mathrm{CaF}_{2},{ }^{75,76}$ due to the particularly interesting and intense emission of Tm at $800 \mathrm{~nm}$.

The convenience of this approach has been proved comparing deep-tissue imaging obtained with organic fluorophores and with $\mathrm{NaYF}_{4}: 0.1 \% \mathrm{Tm}^{3+}, 20 \% \mathrm{Yb}^{3+}$ UCNPs that showed much higher resolution and penetration. ${ }^{71}$ However, a fundamental requirement for a high resolution imaging is a high brightness that makes mandatory the enhancement of the generally quite low QY of these particles. One of the most elegant and efficient strategies is the design of core-shell structures able both to protect the lanthanide ions from nonradiative quenching processes and to promote energy transfer among the different components to favour the upconversion mechanisms. 
Liu et al. ${ }^{78}$ synthesised hexagonal $\mathrm{NaYF}_{4}: \mathrm{Yb}, \mathrm{Er} @ \mathrm{NaYF}_{4}$ core-shell NPs with different shell thickness and investigated the correlation with UC emission intensity for colloidal dispersion in cyclohexane. They found that the green UC intensity is enhanced with increasing shell thickness until a factor of 12 with respect to the core-only NPs. Very similar species were obtained by Chen, Prasad and co-workers ${ }^{79}$ with a systematic synthetic approach that yields size-tunable and monodispersed pure hexagonal phase $\mathrm{NaYbF}_{4}: \mathrm{Tm}^{3+} 0.5 \%$ NPs when doped with

$1030 \%$ of Gd ions. These nanophosphors undergo an impressive enhancement (350 fold) of the upconversion Tm luminescence $(800 \mathrm{~nm})$ when covered with a $2 \mathrm{~nm}$ shell of $\mathrm{NaYF}_{4}$ that is able to well suppress surface quenching mechanisms. The $\mathrm{NaYbF}_{4}: \mathrm{Gd}^{3+} 30 \% \mathrm{Tm}^{3+} 0.5 \% @ \mathrm{NaYF}_{4} \mathrm{NPs}$ showed high contrast 15 ability when tested in in vivo whole-body and ex vivo imaging of nude mice. Many are the examples of multishell structures and we will discuss some other of them hereafter but we would also stress that they can involve different materials. For examples an outer gold shell of proper thickness on silica coated hexagonal phase $\mathrm{NaYF}_{4}: \mathrm{Yb}$,Er NPs presents a plasmonic absorption able to transfer excitation energy at $980 \mathrm{~nm}$ inducing an enhancement of all the UC emission peaks. ${ }^{80}$

The UCNPs can also be part of more complex structures as proposed by Shi and co-workers ${ }^{77}$ that have included NaY$\mathrm{F}_{4}: \mathrm{Yb}, \mathrm{Tm}_{\mathrm{N}} @ \mathrm{NaYF}_{4} @ \mathrm{SiO}_{2}$ nanoparticles of circa $33 \mathrm{~nm}$ of diameter in hollow mesoporous silica shell spheres (Fig. 2). They have then exploited the spectral overlap of the emission of the UCNPs with the absorption of tris(4,7-diphenyl-1,10phenanthroline) ruthenium(II) $\left(\left[\mathrm{Ru}(\mathrm{dpp})_{3}\right]^{2+}\right)$, also present in the cavity, to obtain selective hypoxia imaging in vivo under NIR excitation. Simpler systems have been proposed to detect also biologically relevant metal ions, such as $\mathrm{Ca}^{2+}$ or $\mathrm{Zn}^{2+}$ ex vivo ${ }^{81}$ and in vivo (zebrafish). ${ }^{82}$ In these examples the UCNPs were decorated on the surface with specific receptors suitable

35 to take part in energy transfer processes with the nanoparticle and signal the target binding.

To make the optical imaging more and more specific many groups have investigated the possibility of introducing a target component for example folic acid (FA) to recognize tumor cells

40 or an antibody able to interact with the antigens on gramnegative bacteria. With two different ligands - oleic acid (OA) and aminohexanoic acid (AA) - Li and co-workers ${ }^{83}$ succeeded in the hydrothermal synthesis of high-quality water-soluble upconversion nanocrystals bearing appropriate functional 45 groups using a one-step synthetic strategy. The OA/AA molar ratio allowed us to optimize water dispersibility and provided amino groups for conjugation to folic acid. Their FA surface derivatized $\mathrm{LaF}_{3}: 20 \% \mathrm{Yb}, 1 \% \mathrm{Tm}$ NPs were successfully applied in the lymphatic capillary bioimaging of small animals with a high signal-to-noise ratio (Fig. 3).

Zhang and co-workers ${ }^{84}$ have taken advantage of the high photostability of antibody-based bacterial targeting $\mathrm{NaYF}_{4}: \mathrm{Y}$ b,Er NPs to uniformly label and long term monitor $E$. coli in infected dendritic cells in vitro. The same strategy can also be used to reach a much more difficult target such as glioblastoma in brain. Commercial dyes or contrast agents generally suffer of
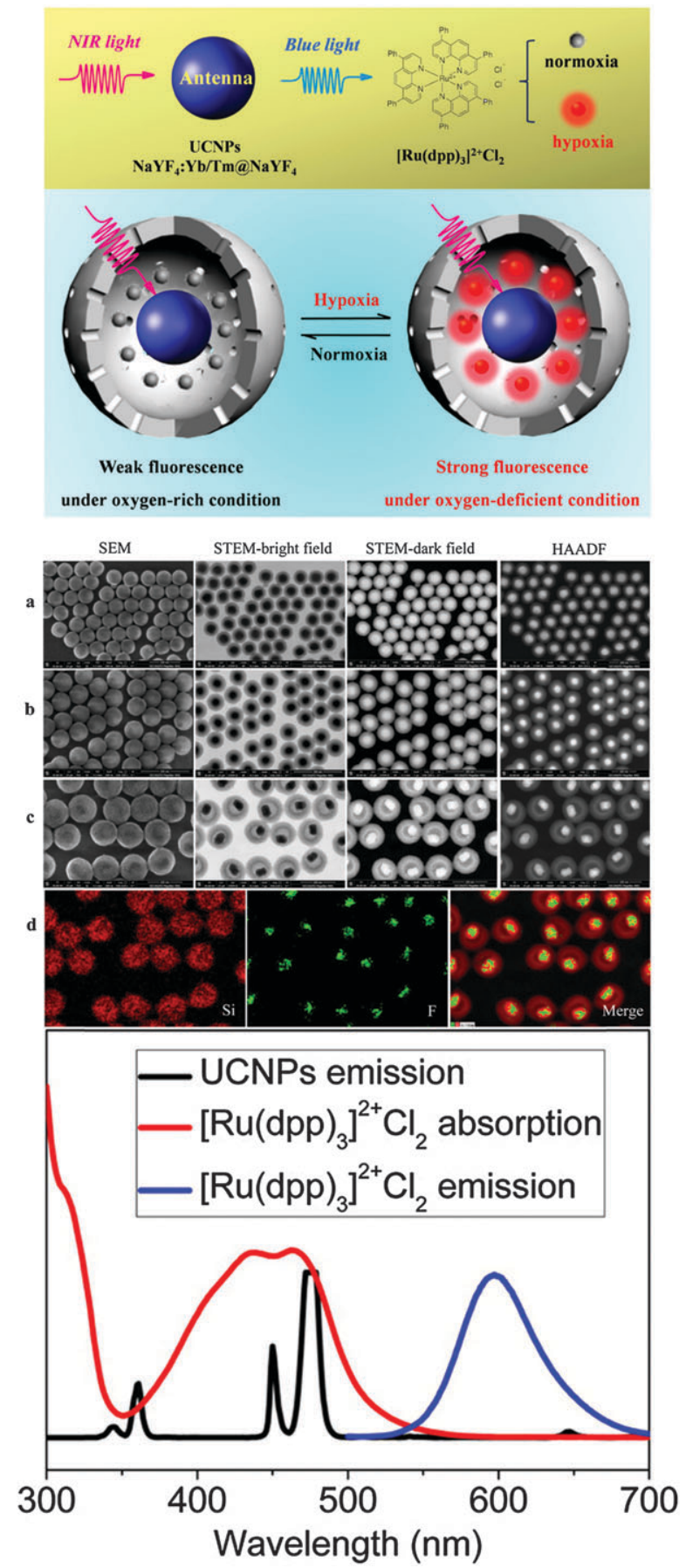

Fig. 2 Schematic illustration of the structure of nanosensors and their sensing to oxygen with a change in luminescence emission (upper). Scanning electron microscopy (SEM), bright/dark field scanning transmission electron microscopy (STEM), and high angle annular dark field (HAADF) images of UCNP@adSiO ${ }_{2}$ (a), UCNPadSiO $a_{2} \mathrm{amSiO}_{2}$ (b), and UCNP@hmSiO 2 (c). (d) Corresponding elemental (F, Si) mappings of UCNPahmSiO 2 (centre). Normalized absorption and emission spectra showing the spectral overlap of the emission of the $\mathrm{NaYF}_{4}: \mathrm{Yb} / \mathrm{Tm}$ NPs with the absorption of the oxygen probe $\left[\mathrm{Ru}(\mathrm{dpp})_{3}\right]^{2+} \mathrm{Cl}_{2}$. Black line: emission spectrum of the nanoparticles under photoexcitation at $980 \mathrm{~nm}$. Red and blue line: absorbance and emission spectrum of $\left[\mathrm{Ru}(\mathrm{dpp})_{3}\right]^{2+} \mathrm{Cl}_{2}$, respectively (bottom). Adapted from ref. 77 Copyright 2014 American Chemical Society.

1 

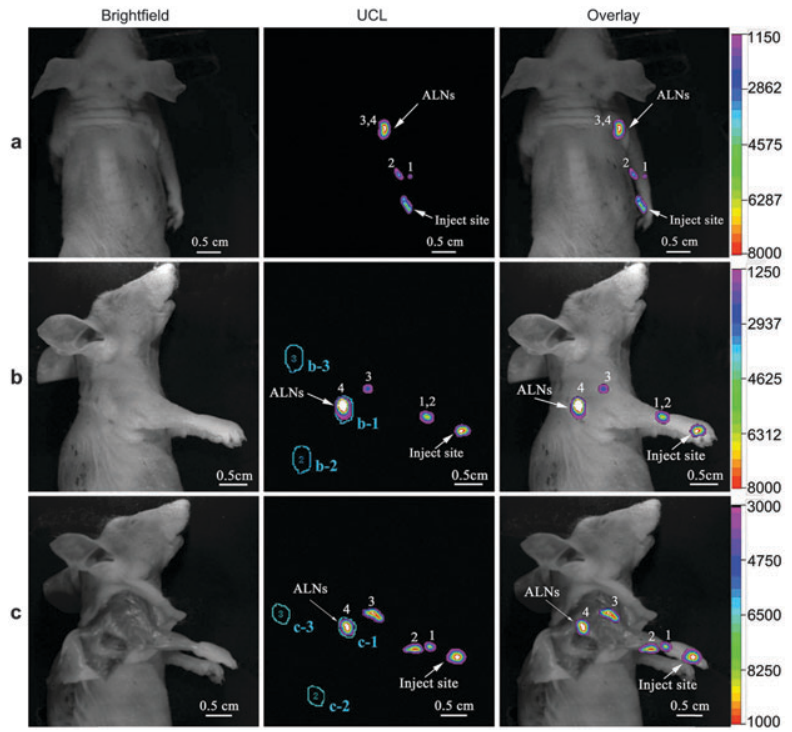

Fig. 3 In vivo lymphatic drainage UCL imaging at $800 \mathrm{~nm}$ was clearly detected at four different draining lymph basins $(1,2,3,4)$ along the right antebrachium of the nude mouse. Detection of upconversion luminescence in the different positions, prostrate (a) or lateral (b) position, after injection of $20 \mathrm{~mL}\left(1 \mathrm{mg} \mathrm{mL}^{-1}\right) \mathrm{LaF}_{3}: \mathrm{Yb}, \mathrm{Er}$ UCNPs into the paw of the nude mouse for $20 \mathrm{~min}$. (c) The lymphatic drainage UCL imaging after removal of skin and fatty tissues was also measured. All images were acquired under the same instrumental conditions (power density of $120 \mathrm{~mW} \mathrm{~cm}{ }^{-2}$ and temperature at $25{ }^{\circ} \mathrm{C}$ on the surface of the mouse). The mean luminescence intensity ROI (blue areas): ROI 1, (specific uptake), ROI 2, (nonspecific uptake) and ROI 3 (background) were selected for the in vivo signal-to-noise ratio (SNR) calculation. Adapted from ref. 83 Copyright 2011 Elsevier Ltd.

poor blood-brain-barrier (BBB) permeability, non-specificity and rapid clearance and $\mathrm{Bu}$, Shi and co-workers ${ }^{85}$ have proposed a targeted, biocompatible system based on UCNPs for the diagnosis and the fluorescence positioning of glioblastoma to guide efficient tumor surgery.

They prepared $\mathrm{NaYF}_{4}: 20 \% \mathrm{Yb} / 2 \% \mathrm{Tm} / 15 \% \mathrm{Gd} @ \mathrm{NaGdF}_{4} \mathrm{NPs}$ capped with amine poly(ethylene glycol)-thiol (PEG) conjugated with Angiopep-2 (ANG) that is specifically able to bind lipopro-

40 tein receptors overexpressed on both BBB and glioblastoma cells, resulting in the ANG-PEG-UCNPs nanocomposites (Fig. 4). These species have the further advantage, thanks to the presence of Gd ions, to be suitable also for MRI investigations making this system a dual-modal nanoprobe. Experi-

45 ments in vivo did not evidence any toxicity while ANG-PEGUCNPs showed very promising results for preoperative diagnosing and intraoperative positioning the brain tumors by MR and NIR-to-NIR UCL fluorescence imaging.

It has to be also mentioned for completeness that the administration method of the imaging agents can co-operate in the targeting process and $\mathrm{Li}$ and co-workers ${ }^{86}$ have shown that the uptake of $\mathrm{NaYF}_{4}$ nanoparticles doped with $\mathrm{Yb}^{3+}$ and $\mathrm{Tm}^{3+}$ in MVF-7 tumours in vivo, following intra-arterial injection, is nearly three-fold higher than that obtained by intra-venous injection for the same species. In the $\mathrm{NaYF}_{4}: \mathrm{Yb}, \mathrm{Tm} @ \mathrm{SiO}_{2} \mathrm{NPs}$ the lanthanide-doped core was synthesized using a solvothermal

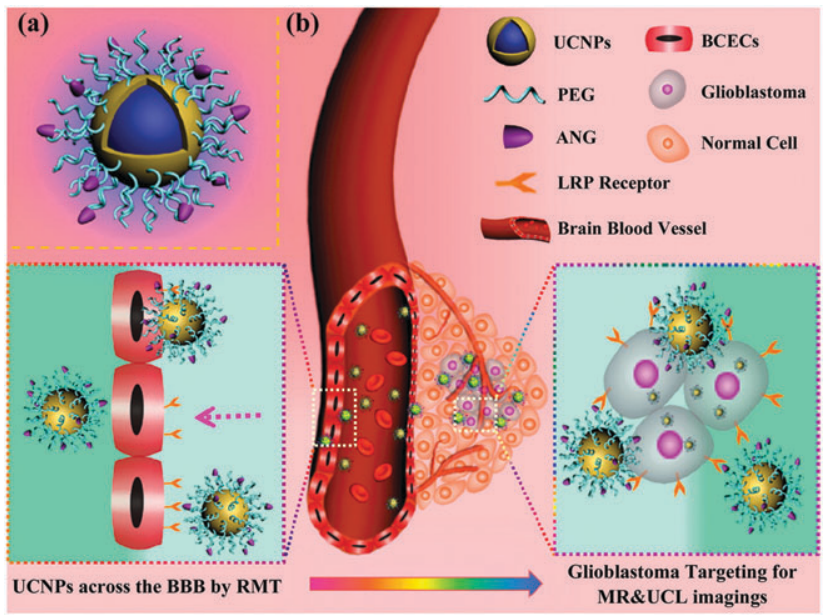

1

Fig. 4 (a) Design of the dual-targeting ANG-PEG-UCNPs. (b) Schematic diagram of the ANG-PEG-UCNPs as the dual-targeting system to cross the BBB and target the glioblastoma via LRP mediated endocytosis, enabling $\mathrm{MR}$ and $\mathrm{UCL}$ imaging of intracranial glioblastoma. Adapted from ref. 85 Copyright 2014 American Chemical Society.

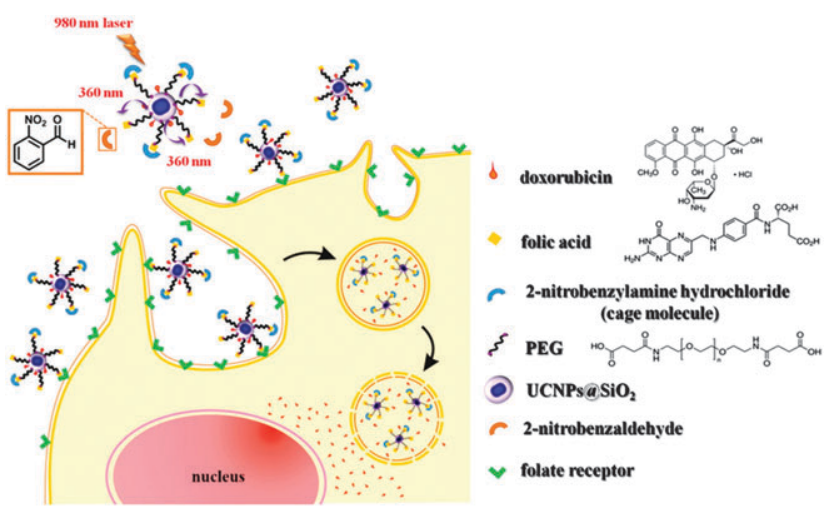

Fig. 5 PEGylated $\mathrm{NaYF}_{4}: \mathrm{Yb}, \mathrm{Tm}_{\mathrm{aSiO}}$ NPs with therapeutic features. Adapted from ref. 87 Copyright 2013 American Chemical Society.

method (average diameter $20 \mathrm{~nm}$ ), and during the silica coating step ethoxysilane functionalized polyethylene glycol (PEG-siloxane) was added into the microemulsion to achieve PEGylation (final hydrodynamic diameter $\sim 65 \mathrm{~nm}$ ).

The implementation of these UC targeted nanolabels with therapeutic features is the last step to go from pure imaging to theranostics. Many research groups are actively investigating different possibilities and most of them take advantage again from NIR light irradiation to trigger targeting, drug release or photosensitizers. ${ }^{21}$

Yeh, Su and co-workers ${ }^{87}$ have proposed PEGylated NaY$\mathrm{F}_{4}: \mathrm{Yb}, \mathrm{Tm} @ \mathrm{SiO}_{2}$ NPs bearing doxorubicin (a chemotherapeutic agent) on the surface, via a disulfide bond that can be cleaved by lysosomal enzymes within the cells (Fig. 5).

Together with the anticancer agent, on the surface folic acid (FA) is also present with masked carboxylic groups via a photocleavable protecting group. In this very complex system the excitation with $980 \mathrm{~nm}$ light induces UC UV emission at 
$1360 \mathrm{~nm}$ that triggers the folic acid cancerous cell targeting by activating the photocleavage reaction of FA protecting agents. The results obtained with these NIR-triggered targeting vehicles in both in vitro and in vivo imaging and in chemotherapeutic efficacy studies evidence the potential of this strategy to improve selective targeting. In this kind of systems the premature release of anticancer drugs before reaching the target can be prevented developing a stimuli-responsive controlled release approach. In particular NIR-remote-controllable nanovectors are highly desir-

Q6 able for in vivo applications and Shi, Bu and co-workers ${ }^{78}$ have proposed a nanoplatform based on mesoporous silica-coated $\mathrm{NaYF}_{4}: 5 \% \mathrm{Tm}, 20 \% \mathrm{Yb} @ \mathrm{NaYF}_{4}$ core-shell UCNPs.

Subsequently the positioning of azobenzene groups (azo) into the mesopores of silica and the loading of doxorubicin

15 yield a system that, upon absorption of NIR light (980 nm), emits UV/Vis photons that are absorbed by the azo molecules inducing a reversible cis-trans photoisomerization. This continuous rotation-inversion movement acts as a molecular impeller that propels the release of the drug (Fig. 6).

The authors were able to demonstrate in in vitro experiments that in these NPs the drug release is only triggered by NIR exposure and the amount of the released drug is highly dependent on the duration and intensity of NIR light exposure. On the other side in slightly simpler systems where $\mathrm{NaGdF}_{4}: \mathrm{Y}$ $\mathrm{b}: \mathrm{Er}(\mathrm{Gd}: \mathrm{Yb}: \mathrm{Er}=78 \%: 20 \%: 2 \%)$ are coated with bovine serum albumin (BSA) loaded with two phototherapy active dye molecules, the NIR light excitation induces killing of cancer cells. ${ }^{79}$ In this case $980 \mathrm{~nm}$ excitation causes a green light emission from UCNPs that is absorbed by the Rose bengal, a photosensitizer for photodynamic therapy (PDT), while the photothermal therapy (PTT) agent IR825 dye can be directly excited at $808 \mathrm{~nm}$. This combined phototherapy demonstrated very good synergistic anti-tumor effects in animal experiments together with the possibility of performing dual modal optical/MR imaging. A similar UCNP-based theranostic nanosystem has been proposed by Liu and co-workers, ${ }^{88}$ in this case a layer-bylayer self-assembly strategy is employed to load multiple strata of Chlorin e6 (Ce6) conjugated polymers onto $\mathrm{Mn}^{2+}$ ion-doped $\mathrm{NaY}(\mathrm{Mn}) \mathrm{F}_{4}: \mathrm{Yb}, \mathrm{Er}$ nanocrystals (Y:Mn: $\mathrm{Yb}: \mathrm{Er}=50: 30: 18: 2$ ).

40 The strong UC emission at ca. $660 \mathrm{~nm}$ under $980 \mathrm{~nm}$ excitation well overlaps the absorption peak of Ce6 that, being a PDT sensitizer, is consequently activated. Again the dual modal optical/MR imaging allows the localization of the nanoprobes that demonstrate remarkably enhanced cell internalization and

45 tumor-homing, thanks to an outer layer of the charge-reversible pH sensitive polymer.

As a last example NIR light has been used to phototrigger drug release from yolk-shell structured mesoporous silica nanocages containing UCNPs $\left(\mathrm{NaYF}_{4}: \mathrm{Tm}^{3+}, \mathrm{Yb}^{3+} @ \mathrm{NaLuF}_{4}\right)$ and a prodrug. ${ }^{81}$ This last species is able to release the drug. after photolysis induced by the upconversion emission light. The authors demonstrated the possibility of photoregulated drug release in living animals with a system characterized by a high drug-loading capacity.

All the examples discussed so far have been based on the $\mathrm{Yb}^{3+}$ sensitizer and therefore involve a laser excitation at
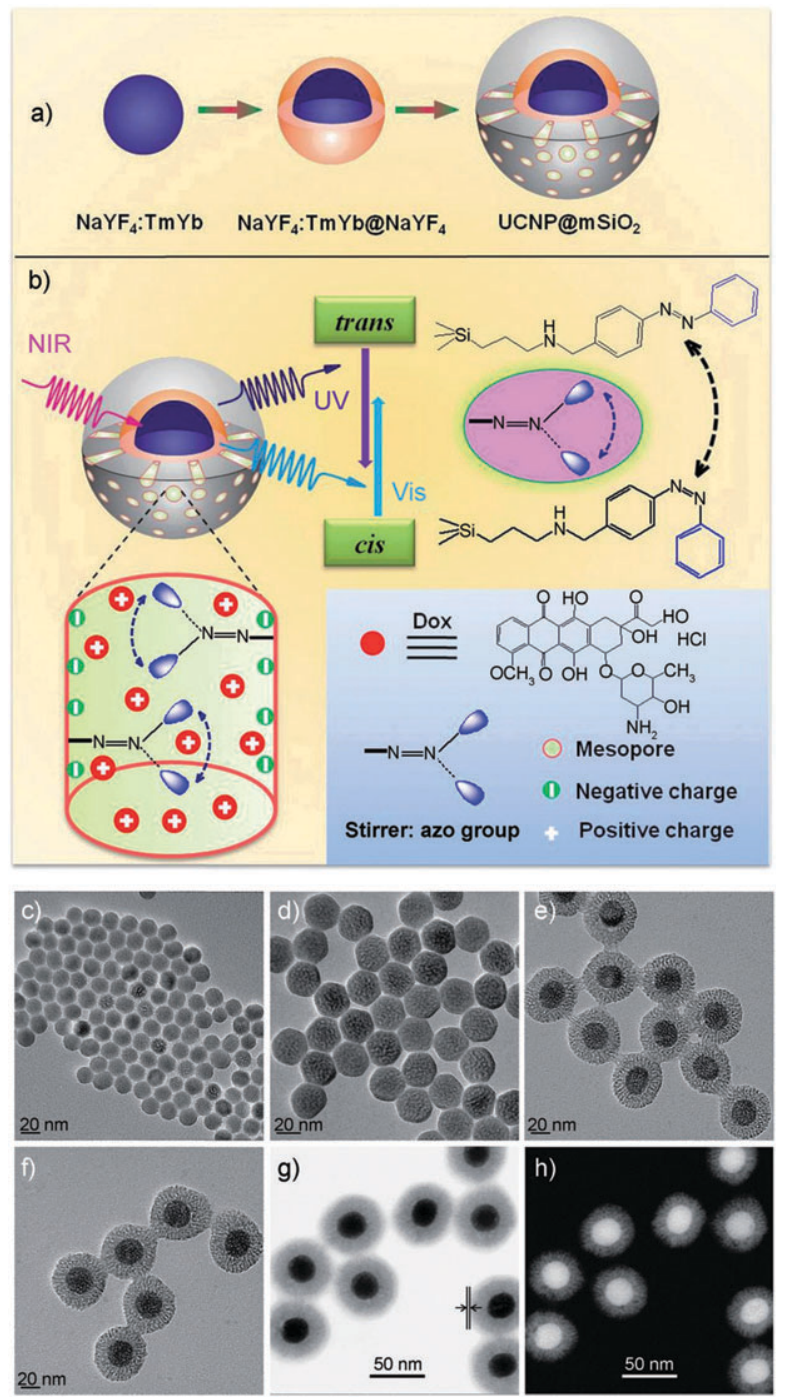

Fig. 6 (a) Synthetic procedure for upconverting nanoparticles coated with a mesoporous silica outer layer. (b) NIR light-triggered Dox release mediated by the upconversion property of UCNPs and trans-cis photoisomerization of azo molecules grafted in the mesopore network of a mesoporous silica layer. TEM images of (c) NaYF $4: T m, Y b$, (d) NaYF $: \mathrm{Tm}_{4}$, $\mathrm{Yb}_{\mathrm{NaYF}}$, (e) $\mathrm{NaYF}_{4}: \mathrm{Tm}$, YbaNaYF $4 \mathrm{amSiO}_{2}$, and (f) NaYF $4: \mathrm{Tm}$, $\mathrm{Yb} \mathrm{NaYF}_{4} @ \mathrm{amSiO}_{2}$-azo. (g) Bright-field and (h) dark-field STEM image of TAT-modified $\mathrm{NaYF}_{4}: \mathrm{Tm}, \mathrm{Yb}_{\mathrm{NNaYF}} \mathrm{amSiO}_{2}$-azo. Adapted from ref. 78 Copyright 2013 Wiley-VCH Verlag GmbH \& Co. KGaA, Weinheim.

$980 \mathrm{~nm}$. Since a few years it has been evidenced and taken into account by many groups that at this wavelength some nonnegligible overheating problems can rise, especially when high power lasers are used. This light energy, in fact, exactly matches the gap between the NIR 1 and the NIR 2 windows, a range where water presents an absorption peak. Therefore, in bioimaging applications, the water absorption at $980 \mathrm{~nm}$ of the excitation beam first of all can be detrimental for depth penetration but, due to the dissipation as heat, it can also cause dramatic tissue damage depending on the laser power and the irradiation time. This is well evidenced by the fact that laser interstitial thermal therapy (LITT), a therapy where tissues
10

1

10

15

20 
1 necrosis is obtained via laser induced local increase of temperature, is often realized by $980 \mathrm{~nm}$ diode lasers. ${ }^{89,90}$ For this reason, in the last few years there is growing attention on systems that can be excited at wavelengths where water does not absorb.

A pioneering computational and experimental study of $\mathrm{He}$ and co-workers ${ }^{91}$ has evidenced that $\mathrm{NaYbF}_{4}: \mathrm{Yb}^{3+} / \mathrm{Tm}^{3+}$ UCNPs encapsulated in a PEG derivative are suitable for in vivo whole body imaging of nude mouse under an excitation wavelength of

$10915 \mathrm{~nm}$. Moreover, the study of the temperature distribution on mouse skin after various irradiation times showed that there is a significant difference in the temperature rise with respect to $980 \mathrm{~nm}$ excitation. A careful comparison of the in vivo data and of the performance of the NPs in phantom tissue revealed that the excitation at 915 was advantageous in terms of depth penetration and minimal excitation power density required, with a consequent much reduced overheating. Other excitation wavelengths have been proposed, for example for LiY$\mathrm{F}_{4}: \mathrm{Er}^{3+} 10 \%$ that under $1490 \mathrm{~nm}$ low power light present a total UC QY higher than the one of $\mathrm{NaYF}_{4}: 20 \% \mathrm{Yb}^{3+} / 2 \% \mathrm{Er}^{3+}$ nanocrystals under excitation at $980 \mathrm{~nm} .^{92}$

More recently, however, it has been evaluated that an excitation matching the local minimum of water absorption spectra at around $800 \mathrm{~nm}$ could be considered an ideal one, inducing the least damage to the biological environment. To this goal UCNPs have been carefully engineered and thanks to cascade sensitization and $\mathrm{Nd}$ ion doping, it has been possible to prepare stable and highly emitting systems under excitation at $800 \mathrm{~nm}$. $\mathrm{Nd}^{3+}$ has, in fact, a large absorption cross section at this wavelength, which presents the double advantage to be in a range where water and other biomolecules have the minimum adsorption and to be covered by commercial easily available and low cost CW lasers with good performance. In a first example Han and co-workers ${ }^{93}$ have found that first of all, due to the rather complicated excited $\mathrm{Nd}^{3+}$ states, its doping concentration needs to be optimized for each combination. They showed that $\beta-\mathrm{NaYF}_{4}: 1 \% \mathrm{Nd}, 30 \% \mathrm{Yb}, 0.5 \% \mathrm{Tm}$ NPs presented the best balance among the component but still a low UC efficiency. Taking into account the high surface to volume ratio in nano-objects and the consequent greater surface quenching risk, it is essential to prevent these deactivations via core-shell strategy. They grew an inert $\beta-\mathrm{NaYF}_{4}$ layer on the UCNP core for a total average diameter of $29 \mathrm{~nm}$ and a circa 20 -fold enhancement of the emission yield. The final result was

45 a much lower heating effect of water under $800 \mathrm{~nm}$ excitation and a comparable UC luminescence intensity with other similar systems. In this case the tri-dopant UCNPs present all the $\mathrm{Ln}^{3+}$ in the core while the shell is un-doped. A different strategy was followed by Yan, Sun and co-workers ${ }^{94}$ that distributed the three components ( $\mathrm{Nd}, \mathrm{Yb}$ and Er ions) in two couples: $\mathrm{Nd} / \mathrm{Yb}$ in the shell and $\mathrm{Yb} / \mathrm{Er}$ in the core, both layers of the $\mathrm{NaGdF}_{4}$ matrix. In their investigation they found that $\mathrm{Nd}^{3+}$ is an irreplaceable sensitizer in order to have an efficient excitation at circa $800 \mathrm{~nm}$, and that the emission intensity of the UCNPs increases with their increasing concentration but at high doping degrees $(>10 \%)$ there are two detrimental effects:
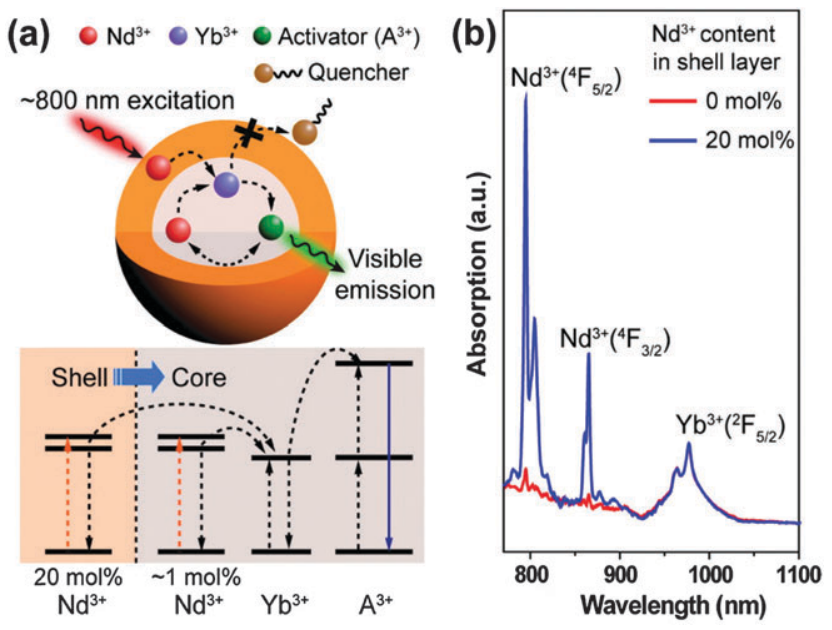

1

Fig. 7 (a) Schematic design (top) and a simplified energy level diagram (bottom) of a core-shell nanoparticle for photon upconversion under $800 \mathrm{~nm}$ excitation. $\mathrm{Nd}^{3+}$ ions doped in the core and shell layers serve as sensitizers to absorb the excitation energy and subsequently transfer it to $\mathrm{Yb}^{3+}$ ions. After energy migration from the $\mathrm{Yb}^{3+}$ ions to activator ions, activator emission is achieved via the $\mathrm{Nd}^{3+}$-sensitization process. (b) NearIR absorption spectra of $\mathrm{NaYF}_{4}: \mathrm{Yb} / \mathrm{Nd}(30 / 1 \%)$ nanoparticles coated with an inert $\mathrm{NaYF}_{4}$ shell or an active $\mathrm{NaYF}_{4}: \mathrm{Nd}(20 \%)$ shell. The absorption spectra were normalized at $976 \mathrm{~nm}$ for comparison. Adapted from ref. 95 Copyright 2013 American Chemical Society.

self-quenching and $\mathrm{Nd}^{3+}$ quenching of the Er emission. To minimize these drawbacks $\mathrm{Nd}^{3+}$ and $\mathrm{Er}^{3+}$ were separated into two different layers and the co-doping with $\mathrm{Yb}^{3+}$ of both of them ensured an efficient sensitizer-activator energy transfer. In this design the activator is shielded and protected by the surface quenching but it is also kept far from the $\mathrm{Nd}$ ions to decrease possible energy back-transfer processes. This strategy has yielded an UC emission efficiency suitable for in vivo imaging of nude mouse under $800 \mathrm{~nm}$ excitation and without overheating side effects. Liu and co-workers ${ }^{95}$ have proposed another distribution of almost the same components in order to further enhance the ET efficiency pushing further the doping degree of $\mathrm{Nd}^{3+}$. They used the $\mathrm{NaYF}_{4}$ matrix to prepare coreshell $\mathrm{NaYF}_{4}: \mathrm{Yb} / \mathrm{Tm} / \mathrm{Nd} @ \mathrm{NaYF}_{4}: \mathrm{Nd} \mathrm{NPs}$ with $2 \% \mathrm{Nd}$ in the core and $20 \mathrm{~mol} \%$ of total $\mathrm{Nd}^{3+}$ considering also that the active shell is present (Fig. 7).

The integrated emission intensity increased of more than 400 times adding the $\mathrm{Nd}^{3+}$ layer coating on the core, a better result in comparison with the ones obtained with core-shell systems with an inert outer shell. Nevertheless, the energy backtransfer was still a challenge and Yao, Ma, Zhao, Gu and coworkers ${ }^{96}$ have carefully investigated the possibility to further reduce this quenching effect and maximize the concentration of the Nd sensitizer. They started from the same structure and components of the previous example but they implemented the system adding a second spacer-layer between the core (containing the $\mathrm{Yb}^{3+}$ and the activator ions) and the outer shell, presenting the $\mathrm{Nd}$ in the matrix (around 90\% of the concentration) and doped with $\mathrm{Yb}^{3+}$. The intermediate layer acts as a shield to prevent the energy back-transfer increasing the distance between $\mathrm{Nd}^{3+}$ and $\mathrm{Er}^{3+}$ and contains a certain amount of 

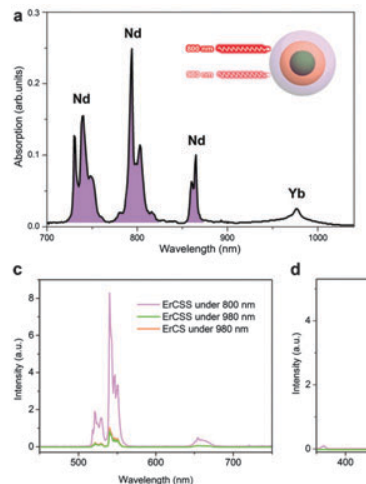
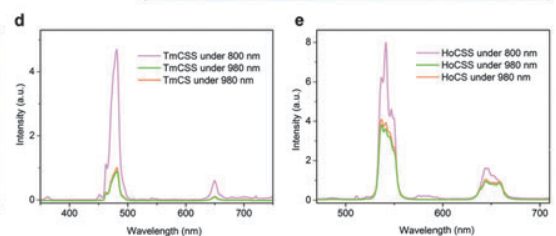

Fig. 8 Photoluminescence comparison of $\mathrm{Nd}$-sensitized quenchingshield sandwich UCNPs (exc. $800 \mathrm{~nm}$ ) and conventional Yb-sensitized core-shell UCNPs (exc. $980 \mathrm{~nm}$ ). (a) Absorption spectra in hexane (20 mg $\mathrm{mL}^{-1}$ ). (b) Luminescence photographs of UCNPs with different activator ions $\left(\mathrm{Er}^{3+}, \mathrm{Tm}^{3+}\right.$, and $\mathrm{Ho}^{3+}$ ) excited at 800 (top) and $980 \mathrm{~nm}$ (bottom) with $0.5 \mathrm{~W} \mathrm{~cm}^{-2}$ diode lasers. (c) UC emission spectra of the ErCSS nanoparticles (excitation at 800 and $980 \mathrm{~nm}$ ) and the ErCS nanoparticles (excitation at $980 \mathrm{~nm})$. (d) UC emission spectra of $\mathrm{NaYF}_{4}: 20 \% \mathrm{Yb}, 0.5 \% \mathrm{Tm}$ (aNaY$\mathrm{F}_{4}: 10 \% \mathrm{Yb}$ aNaNdF $4: 10 \% \mathrm{Yb}$ (TmCSS) nanoparticles (excitation at 800 and $980 \mathrm{~nm}$ ) and $\mathrm{NaYF}_{4}: 20 \% \mathrm{Yb}, 0.5 \% \mathrm{TmaNaYF}_{4}$ (TmCS) nanoparticles (excitation at $980 \mathrm{~nm}$ ). (e) UC emission spectra of $\mathrm{NaYF}_{4}: 20 \% \mathrm{Yb}, 0.5 \% \mathrm{Ho@NaY-}$ $\mathrm{F}_{4}: 10 \% \mathrm{YbaNaNdF}_{4}: 10 \% \mathrm{Yb}$ (HoCSS) nanoparticles (exc. at 800 and $980 \mathrm{~nm}$ ) and $\mathrm{NaYF}_{4}: 20 \% \mathrm{Yb}, 0.5 \% \mathrm{HoONaYF}_{4}$ (HoCS) nanoparticles (excitation at $980 \mathrm{~nm}$ ). Note that the ErCSS, TmCSS, and HoCSS samples were synthesized under the same conditions and the ErCS, TmCS, and HoCS samples were prepared by using the same synthetic method. All the above samples were dispersed in cyclohexane at the same concentration $\left(20 \mathrm{mg} \mathrm{mL}^{-1}\right.$ ). The power density of both lasers is $0.5 \mathrm{~W} \mathrm{~cm}^{-2}$. Adapted from ref. 96 Copyright 2009 Wiley-VCH Verlag GmbH \& Co. KGaA, Weinheim

$\mathrm{Yb}^{3+}$ ions to ensure the migration of the direct energy transfer from the sensitizer to the activator.

The authors investigated the influence of the interlayer thickness on the final UC emission intensity and they have found the best balance of the back and forth ET was obtained for a $1.5 \mathrm{~nm}$ one. The final $\mathrm{NaYF}_{4}: \mathrm{Yb}_{0.2}, \mathrm{Er}_{0.02} @ \mathrm{NaYF}_{4}: \mathrm{Yb}_{0.1} @$ $\mathrm{NaNdF}_{4}: \mathrm{Yb}_{0.1} \mathrm{NPs}$, or the corresponding ones bearing $\mathrm{Tm}^{3+}$ or $\mathrm{Ho}^{3+}$ as activators, under $800 \mathrm{~nm}$ excitation, have UC luminescence higher than the corresponding $\mathrm{NaYF}_{4}: \mathrm{Yb}_{0.2}, \mathrm{Er}_{0.02} @ \mathrm{NaYF}_{4}$ NPs sensitized directly via $\mathrm{Yb}^{3+}$ (Fig. 8).

Also in this case, the evaluation of the ability of the UCNPs to penetrate tissue by in vivo imaging of nude mouse revealed for the $\mathrm{Nd}^{3+}$ sensitized NPs excited at $800 \mathrm{~nm}$ a higher penetration depth and a much lower undesirable overheating than the 980 nm-excited $\mathrm{Yb}^{3+}$ sensitized NPs. These last examples involve highly engineered UCNPs with optimized excitation features but have the partial drawback that the emission light is mostly in the visible range of the spectrum. A very promising strategy that is now extensively explored to overcome also this problem is to exploit lanthanide doped NPs for DS emission, a very interesting topic that needs a separate discussion.

\subsection{DSNPs: Stokes-emitting NPs in the NIR-1 and NIR-2 regions}

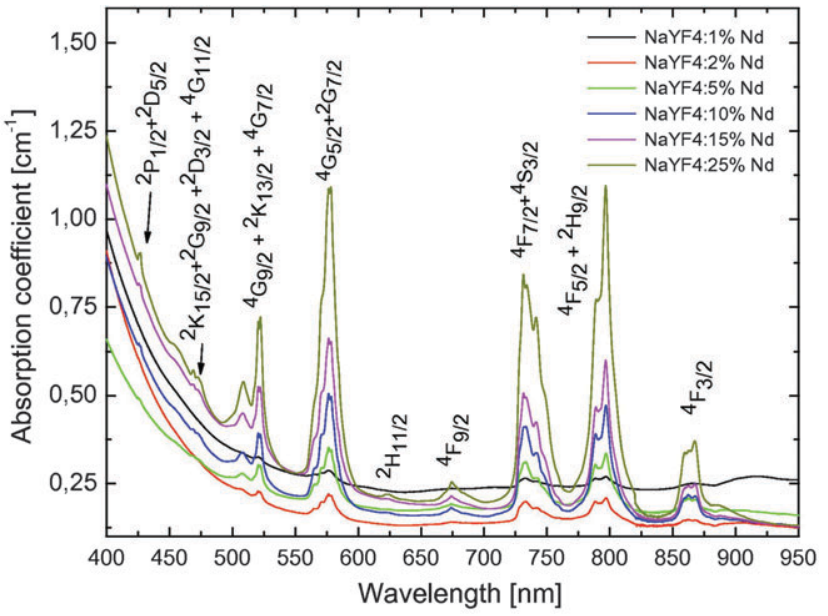

1

Fig. 9 Room temperature absorption spectra of $\mathrm{NaYF}_{4}$ colloidal nanocrystals doped with various amounts of $\mathrm{Nd}^{3+}(1,2,5,10,15$ and $25 \%)$. The bands are assigned to the transitions from a ground state ${ }^{4} I_{9 / 2}$ to the indexed excited ones. Adapted from ref. 97 Copyright 2011 Elsevier Ltd.

significant visible emission under UV excitation, that is to say $\mathrm{Sm}^{3+}, \mathrm{Eu}^{3+}, \mathrm{Tb}^{3+}$ and $\mathrm{Dy}^{3+}$. However, for in vivo imaging, the need of having both the excitation and detection wavelength in the biological optical transparency window has led to the choice of $\mathrm{Nd}^{3+}$ as an emitting unit.

In fact, as it can be seen from Fig. $9, \mathrm{Nd}^{3+}$ can be conveniently excited in the NIR-1 region, and in particular at around $800 \mathrm{~nm}$, where this ion has a relatively high good absorption cross section.

At the same time, the luminescence spectrum of $\mathrm{Nd}^{3+}$ ions presents three possible transitions $\left({ }^{4} \mathrm{~F}_{3 / 2} \rightarrow{ }^{4} \mathrm{~F}_{9 / 2}\right.$ in the NIR-1 and ${ }^{4} \mathrm{~F}_{3 / 2} \rightarrow{ }^{4} \mathrm{~F}_{11 / 2}$ and ${ }^{4} \mathrm{~F}_{3 / 2} \rightarrow{ }^{4} \mathrm{~F}_{13 / 2}$ in NIR-2), at around 900, 1050 and $1300 \mathrm{~nm}$, respectively, with luminescence quantum yields that in NPs, summing up the three contributions, can be as large as $80 \%$ (Fig. 10). ${ }^{95,97,98}$

The luminescence of $\mathrm{Nd}^{3+}$ doped NPs has been reported in the last few years by several groups for core and core-shell NPs.

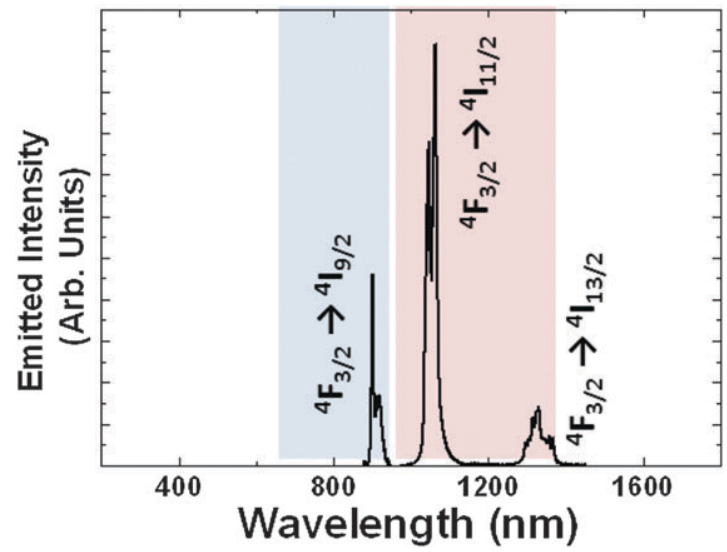

Fig. 10 Emission spectrum of a colloidal solution of $\mathrm{Nd}^{3+}: \mathrm{LaF}_{3} \mathrm{NPs}$ as obtained after $808 \mathrm{~nm}$ optical excitation. NIR-1 (blue) and NIR-2 (pink) biological windows are schematically labeled. Adapted from ref. 31 Copyright 2014 Wiley-VCH Verlag GmbH \& Co. KGaA, Weinheim.
In the DS emitting nanocrystals the co-doping strategy is rarely adopted and the $\mathrm{Ln}^{3+}$ ions commonly used are those presenting 
1 The optical properties of $\mathrm{YF}_{3}: \mathrm{Nd}^{3+} \mathrm{NPs}$, prepared by solvothermal decomposition of trifluoroacetate precursors in high boiling point solvents, have been investigated by Tan et al. ${ }^{99}$ These NPs, that have a size of about $20 \mathrm{~nm}$, showed a fluorescence quantum yield and an excited state lifetime that was highly dependent on the concentration of the dopant. In particular, the luminescence spectra and the excited state decays have been recorded for concentrations ranging from $0.25 \mathrm{~mol} \% \leq$ $\mathrm{Nd} \leq 5 \mathrm{~mol} \%$. In this range, the quantum yield dropped from about $75 \%$ to less than $5 \%$, while, at the same time, the average lifetime passed from 588 to $6 \mu \mathrm{s}$. This is a behaviour that is common to all $\mathrm{Nd}^{3+}$ doped NPs and can be attributed to two concomitant energy transfer processes, i.e., cross relaxation (CR) and energy migration (EM), whose overall efficiency varies with the square of dopant concentration.

In $\mathrm{CR}$, the luminescent ${ }^{4} \mathrm{~F}_{3 / 2}$ excited state transfers only part of its energy to a $\mathrm{Nd}^{3+}$ ion in the ${ }^{4} \mathrm{~F}_{9 / 2}$ ground state to give two ions in the ${ }^{4} \mathrm{~F}_{15 / 2}$ state, leading to a decrease of the luminescence, according to the following scheme:

$$
{ }^{4} \mathrm{~F}_{3 / 2}+{ }^{4} \mathrm{~F}_{9 / 2} \rightarrow 2{ }^{4} \mathrm{~F}_{15 / 2}
$$

In the case of energy migration, all the energy of a ${ }^{4} \mathrm{~F}_{3 / 2}$ state is transferred from a $\mathrm{Nd}^{3+}$ ion to a neighbouring one and so on. In this way the excitation energy is not lost; however, this process increases the probability that a $\mathrm{Nd}$ ion present at (or close to) the surface could be excited; since these ions can be efficiently quenched by surface effects (ligands, solvent molecules, and surface defects), they behave as an energy sink, thus greatly decreasing the overall luminescence quantum yield. The negative effects of energy migration can be minimized, as also discussed for UCNPs, with the epitaxial growth of an undoped outer shell that can efficiently shield these $\mathrm{Nd}^{3+}$ ions from surface quenchers. ${ }^{100}$ This is a very interesting strategy, which has been followed by different authors. For example, Xie et al. ${ }^{101}$ have investigated $\mathrm{Nd}^{3+}$ doped $\mathrm{LaF}_{3}$ nanocrystals prepared by a surfactant-free aqueous solution route. In this case, the authors showed that core-shell NPs prepared by coating the same NPs with an outer $\mathrm{LaF}_{3}$ shell showed a 60\% intensity increase at $1056 \mathrm{~nm}$ with respect to the uncoated NPs.

In this context, another very interesting example has been proposed by Prasad and co-workers that synthesised small core-shell oleate-capped core-shell $\mathrm{NaGdF}_{4}: \mathrm{Nd}^{3+} / \mathrm{NaGdF}_{4}$ $\mathrm{NPs}^{100}$ (average diameter $15 \mathrm{~nm}$ ) with efficient NIR-to-NIR 45 down shifting PL $\left(\lambda_{\mathrm{ex}}=740 \mathrm{~nm}, \lambda_{\mathrm{em}}=850-900 \mathrm{~nm}\right)$ for in vitro and in vivo imaging. ${ }^{100}$ They adapted a previously reported synthetic method bearing hexagonal-phase core-shell $\mathrm{NaYF}_{4}: \mathrm{Yb}, \mathrm{Tm} @ \mathrm{NaYF}_{4}: \mathrm{Yb}, \mathrm{Er}$ nanocrystals containing Tm and Er in the core and in the shell, respectively. ${ }^{102}$ In this case they take advantage of a $\mathrm{NaGdF}_{4}$ shell covering a $\mathrm{NaGdF}_{4}: \mathrm{Nd}^{3+}$ core to suppress non-radiative recombination processes at the NP surface. More in detail, to optimize the overall signal of the NPs, the authors have adopted two sequential steps. The first one was based on the optimization of the brightness of the $\mathrm{NaGdF}_{4}: \mathrm{Nd}^{3+}$ core upon varying the $\mathrm{Nd}^{3+}$ concentration. In particular, they found that in hexane the photoluminescence quantum yields of these nanostructures doped with a 3, 6, 10, and $15 \%$ of the emitting ion were estimated to be about $22,9.4$, 5.0 and $3.6 \%$, respectively, suggesting that the highest brightness (that has to take into consideration also the absorbance of $\mathrm{Nd}^{3+}$ and thus is concentration) could be obtained with a $3 \%$ doping degree. The authors also showed that lowering even further the concentration of the dopant to $1 \%$ could not take to an additional increase of the quantum yield. Once optimizing the $\mathrm{Nd}$ concentration, the authors grew a $2 \mathrm{~nm}$ thick shell of $\mathrm{NaGdF}_{4}$ obtaining in this way NPs showing a QY as high as $40 \%$. The effect attributable to the shell is thus a $86 \%$ increase of the luminescence, very close to the effects previously discussed. A parallel increase in the excited state lifetime from 190 to $240 \mu \mathrm{s}$ was also observed. For biological applications, these NPs were then transferred to the aqueous phase by the ligandfree method; in this condition however the QY resulted to be two times lower (and the excited state accordingly shorter) because of the multi-phonon deactivation process due to the high energy of the $\mathrm{O}-\mathrm{H}$ oscillators, suggesting that the shell used for these NPs does not provide an absolute protection for the $\mathrm{Nd}^{3+}$ ions against surface effects.

The NIR-to-NIR bioimaging was successfully demonstrated in vivo through visualization of the emission at $900 \mathrm{~nm}$ upon Xe lamp excitation from a band-pass optical filter $(710-760 \mathrm{~nm}) .{ }^{100}$ In this experiment the NPs were injected subcutaneously into a mouse and the luminescence imaging was measured using a CCD camera (Fig. 11). A bright emission was clearly visible at the injection point, while almost no autofluorescence was observed elsewhere, resulting in a high contrast image.

An exhaustive and rigorous study on the optimization of the best conditions for efficient bioimaging with DS nanoparticles based on $\mathrm{Nd}^{3+}$ ions has been performed by Jaque and coworkers. ${ }^{31,103,104}$ They synthesized $\mathrm{Nd}^{3+}$ doped $\mathrm{LaF}_{3}$ nanocrystals having a diameter of $15 \mathrm{~nm}$ and a size dispersion close to $30 \%$ with different concentrations of the dopant to obtain the highest brightness, reached with a $15 \%$ Nd concentration. ${ }^{104}$ An important subject of the study was to verify which of the three transitions observed in the luminescence spectrum, at around 900, 1050 and $1300 \mathrm{~nm}$, could give the best signal under normal imaging conditions. ${ }^{31}$ The parameters to be considered are

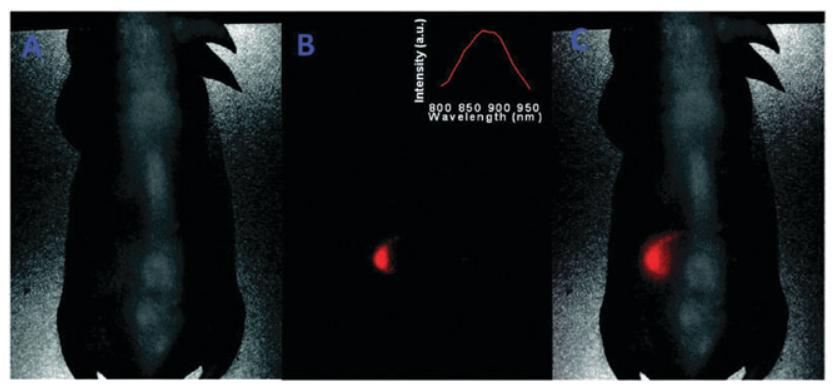

Fig. 113.5 in vivo whole-body image of a nude mouse subcutaneously injected with ligand-free $\left(\mathrm{NaGdF}_{4}: 3 \% \mathrm{Nd}^{3+}\right) / \mathrm{NaGdF}_{4}$ nanocrystals: (A) bright field image, (B) PL image, and (C) superimposed image (bright field image and spectrally unmixed PL image). (inset) Spectra of the NIR PL signals. Adapted from ref. 100 Copyright 2012 American Chemical Society. 
1 three: (i) the relative intensity of each transition; (ii) the sensitivity of the detectors at the different wavelengths, and (iii) the transparency of tissues at each wavelength. As far as the relative intensity is concerned, for the system under study a relative intensity of $0.25,0.60$ and 0.15 , respectively, was reported, while the sensitivity of the InGaAs array typically used for these experiments is, in arbitrary units, 0.4, 0.8 and 0.8 , respectively. The tissue transparency was instead evaluated measuring the intensity transmitted by layers of phantom (mimicking the optical properties of human skin) of different thicknesses. The obtained extinction coefficients, which take into account both absorption and scattering processes, are 7.9, 6.0 and 5.0. Summing up all terms, the ${ }^{4} \mathrm{~F}_{3 / 2} \rightarrow{ }^{4} \mathrm{~F}_{11 / 2}$ transition at around $1050 \mathrm{~nm}$, in the second

15 biological window, was shown to be the best for subtissue imaging, also if compared to the traditionally used ${ }^{4} \mathrm{~F}_{3 / 2} \rightarrow$ ${ }^{4} \mathrm{~F}_{9 / 2}$ at $910 \mathrm{~nm}$ (first biological window). In addition, since in in vivo optical imaging the spatial resolution is restricted mainly by scattering processes, the use of the emission in NIR-2 could also be beneficial from this point of view. High tissue penetration ( $c a .1 \mathrm{~cm})$, observing the luminescence spot originating from injection points performed at different depth in mice, was then obtained in in vivo experiments.

Interestingly, the same group has also demonstrated that these $\mathrm{Nd}^{3+}$ doped core-shell $\mathrm{LaF}_{3}$ NPs can be used as subtissue thermal sensors. ${ }^{104}$ In fact, upon increasing the temperature, a gradual, small shift $\left(0.10 \mathrm{~cm}^{-1} \mathrm{~K}^{-1}\right)$ of the band at $864 \mathrm{~nm}$ can be observed, accompanied by a change of the ratio of the intensities observed at 885 and $863 \mathrm{~nm}$. Both these parameters can allow us to measure temperatures with a thermal resolution close to $2{ }^{\circ} \mathrm{C}$. Although this resolution is much lower than the one obtained with other systems, ${ }^{105}$ it is possible to appreciate the temperature increase obtained in a single-beam plasmonicmediated heating experiment, as described in Fig. 12. Gold nanorods were used as heaters, taking profit of their high absorbance at $808 \mathrm{~nm}$, where also the NPs are excited, and the temperature monitored by the NIR $\mathrm{Nd}^{3+}$ emission, evidencing how the temperature induced spectral shift and intensity variation emission bands of the $\mathrm{Nd}^{3+}$ ions can be successfully 0 monitored to estimate the local temperature. In addition, the same kind of $\mathrm{Nd}^{3+}$ doped $\mathrm{LaF}_{3}$ NPs was studied ${ }^{103}$ also for their photo-thermal properties. In this case, however, the NPs were activated with an even larger amount of $\mathrm{Nd}^{3+}$ ions, up to $25 \%$, decreasing in this way the brightness of the system (because of the decrease of the QY observed at high dopant concentration), but largely increasing the ability to generate heat under optical excitation at around $800 \mathrm{~nm}$. In this way, with the same NP it has been possible to generate an increase of the local temperature in a chicken breast tissue, an increase that could be concomitantly monitored via the observed luminescence. This is an interesting proof of principle for the development of theranostic platforms based only on radiation light in the NIR-1 biological window.

A very recent paper by Villa et al. ${ }^{103}$ reported an investigation of $\mathrm{SrF}_{2}: \mathrm{Nd}^{3+} \mathrm{NPs}$ for in vivo imaging in the NIR-2 biological window.

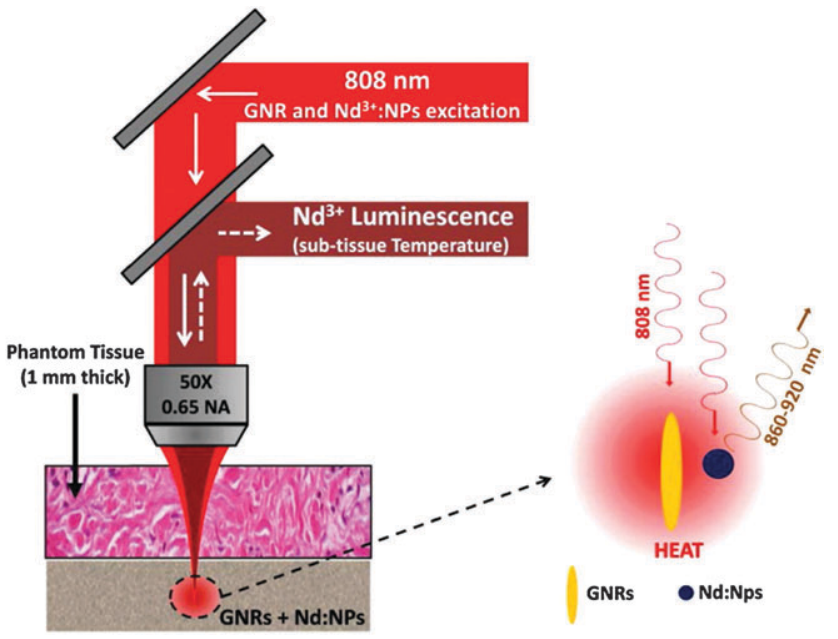

Fig. 12 Schematic representation of the experimental setup used for single-beam sub-tissue-controlled hyperthermia. A $808 \mathrm{~nm}$ laser beam is focused into an aqueous solution containing GNRs (nanoheaters) and $\mathrm{Nd}^{3+}: \mathrm{LaF}_{3}$ nanoparticles (nanothermometers). The solution was placed under a $1 \mathrm{~mm}$ thick phantom tissue. The luminescence of $\mathrm{Nd}^{3+}: \mathrm{LaF}_{3}$ nanoparticles was collected by using the same objective, and the subtissue temperature was extracted from its spectral analysis. Diagram at the right reflects the fact that both GNRs and $\mathrm{Nd}^{3+}: \mathrm{LaF}_{3} \mathrm{NPs}$ were simultaneously excited by the $808 \mathrm{~nm}$ radiation. Adapted from ref. 104 Copyright 2013 American Chemical Society.

The particles were prepared by an environmentally friendly technique, using water as a solvent and relatively low treatment temperatures. Upon excitation at $808 \mathrm{~nm}$ with a diode laser, the emission band at $1340 \mathrm{~nm}$ of the $\mathrm{Nd}^{3+}$ ion was used to measure in vivo and ex vivo optical images of mice after intravenous administration of a colloidal dispersion of the $\mathrm{SrF}_{2}: \mathrm{Nd}^{3+}$ particles.

This investigation showed a negligible overlap between the emission band at $1340 \mathrm{~nm}$ of the $\mathrm{Nd}^{3+}$ ions and the infrared autofluorescence due to the food commonly used to feed the mice. The particles have been found to accumulate mainly in the liver and spleen, as shown in Fig. 13.

$\mathrm{Nd}^{3+}$ doped bimodal $\mathrm{GdF}_{3}$ NPs were also investigated by Sardar et al., ${ }^{106,107}$ and their fluorescence and magnetic properties have been demonstrated. The NPs (average size $\sim 5 \mathrm{~nm}$ ) were prepared by thermal decomposition and subsequently coated with poly(maleic anhydride-alt-1-octadecene), PMAO, to increase the water solubility and the cellular uptake. The absolute QY of the $\mathrm{Nd}^{3+}$ emission in the 850-1400 $\mathrm{nm}$ range for the $\mathrm{GdF}_{3}: \mathrm{Nd}^{3+}$ powders resulted to be $10.2 \pm 1.6 \%$ upon an excitation wavelength of $800 \mathrm{~nm}$ and an excitation power density of $12 \mathrm{~W} \mathrm{~cm}^{-2}$. It is important to note that, differently from other cases, the authors noted a dependence of the QY on the excitation power density. The functionalized NPs form stable colloids and their cellular uptake has been tested on fibroblast cells. By placing the NPs under pig skin of different thicknesses, they could observe a clear signal also at the highest tested thickness $(5 \mathrm{~mm})$. In addition, they also found that the DS QY of these NPs were 2000 times higher than that of UC $\beta-\mathrm{NaYF}_{4}: 20 \% \mathrm{Yb}^{3+} / 2 \% \mathrm{Er}^{3+}$ of the same size. 

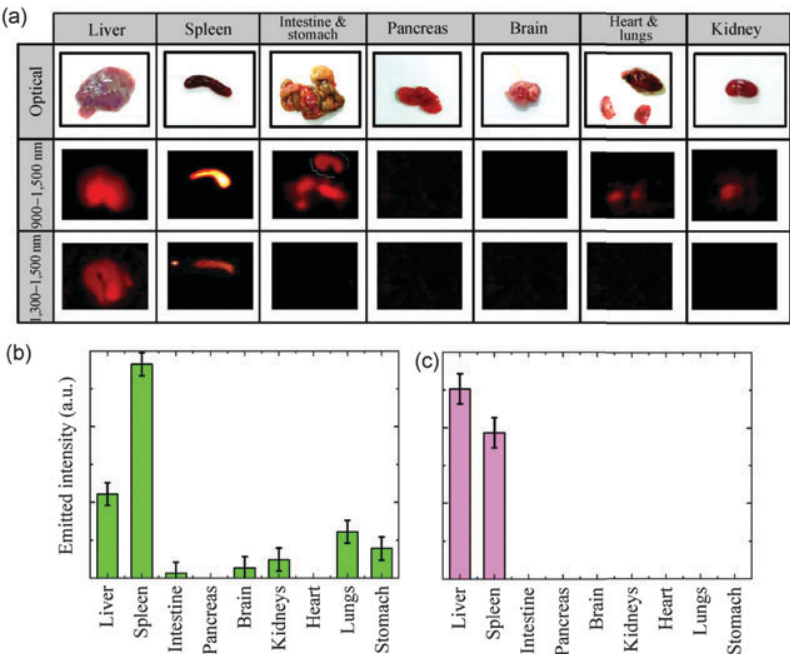

Fig. 13 (a) Optical and fluorescence images in the 900-1500 and 1300$1500 \mathrm{~nm}$ spectral detection ranges of the organs extracted from a sacrificed mouse 1 hour after an intravenous injection of $\mathrm{Nd}: \mathrm{SrF}_{2}$ particles. (b) $900-$ $1500 \mathrm{~nm}$ integrated fluorescence intensity obtained from the different organs. (c) 1300-1500 nm integrated intensity obtained from the different organs. In all cases, the integrated fluorescence intensity has been normalized by the organ weight. Adapted from ref. 103 Copyright 2014 Springer.

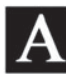

Fig. 14 (A) General strategy to achieve the UC and DS dual-mode luminescence with multi-layer C/S1/S2/S3 $\beta-\mathrm{NGdF}_{4}: \mathrm{Nd} / \mathrm{NaYF}_{4} / \mathrm{NaGdF}_{4}: \mathrm{Nd}, \mathrm{Yb}, \mathrm{Er} / \mathrm{NaYF} 4$ NPs. (B) Proposed energy transfer mechanisms in the multi-layer core-shell NPs. Adapted from ref. 109 Copyright 2013 Macmillan Publishers Limited.

A preference for NIR-to-NIR DSNPs with respect to NIR-toNIR UCNPs for in vivo imaging has been evidenced also by other authors. The advantages of DSNPs are many: first of all, the luminescence quantum yield of these nanostructures is substantially higher. Besides this, the linear dependence of the emitted light on the excitation intensity - differently to what observed for bi-photonic processes - leads to a larger penetration depth and the possible use of light sources with a relatively low density, a feature that decreases possible biological damage. To take profit of all these features for in vivo imaging, but having an additional signal in the visible region for in vitro experiments, Li et al. ${ }^{108,109}$ prepared $\mathrm{NaGdF}_{4}$ based multishell dual mode (UC and DS) NPs. In particular, as schematized in Fig. 14, these NPs are made of one core and three different shells with a C/S1/S2/S3 structure. The two active parts are the core C $\left(\beta-\mathrm{NaGdF}_{4}: \mathrm{Nd}\right)$ and the second shell S2 $\left(\mathrm{NaGdF}_{4}: \mathrm{Nd}, \mathrm{Y}-\right.$ $\mathrm{b}, \mathrm{Er})$ that were designed for DS and UC luminescence, respectively, both at $\lambda_{\mathrm{ex}}=800 \mathrm{~nm}$. Passive shells $\mathrm{S} 1$ and $\mathrm{S} 3\left(\mathrm{NaYF}_{4}\right)$ were added to prevent surface deactivation and, in the case of S1, possible energy transfer processes to the ions in the active shells. C was responsible for the typical Nd centred luminescence, which was conveniently used for the in vivo imaging of a nude mouse upon low excitation intensity, while S2 was responsible for the green fluorescence at $540 \mathrm{~nm}$ used for the in vitro imaging of human lymphocytes cells.

\section{Lanthanide-based nanoparticles as MRI contrast agents}

Since the seminal paper of Lauterbur ${ }^{110}$ in 1973, Magnetic Resonance Imagining (MRI) has rapidly emerged as one of the most powerful routine diagnostic tools in medicine, its strength being based on the non-invasive nature, the high spatial resolution accompanied by tomographic capabilities, and the absence of ionizing electromagnetic radiation.

The principles behind such a technique lie in the combined use of a static magnetic field $B_{0}$ and magnetic field gradients arranged in such a way that the physical space which encloses the sample magnetization (typically originating from water protons) is mapped into a frequency space of resonating spins. The basic radio-frequency pulse schemes used in MRI to manipulate the sample magnetization are sensitive just to the proton spin density, and are generally of limited use in the imaging of complex tissues or organs. More frequently, contrast is enhanced by $T_{1}$ or $T_{2}$ editing techniques, i.e. by using pulse sequences that are sensitive, as well as the spin density, also the longitudinal $\left(T_{1}\right)$ or transverse $\left(T_{2}\right)$ relaxation times of protons. These two time constants come at play whenever the magnetization created by the external field $B_{0}$ is moved away from its equilibrium value. Within a phenomenological approach, the decay of the $z$-magnetization towards its thermal equilibrium value is governed by the longitudinal relaxation time, whereas the same decay relative to the $x$ - and $y$-magnetization components is governed by the transverse relaxation time. For small, rapidly tumbling molecules in the solution state it is found that $T_{1}=T_{2}$ (fast motion limit). 
When the characteristic relaxivity of protons within different domains is not sufficient to provide an informative image, the use of paramagnetic contrast agents (CAs) can lead to a considerable enhancement of the anatomic or pathologic details under investigation. The reason for this enhancement lies in the fact that, when the contrast agents (usually, metal chelates of paramagnetic ions) accumulate in a certain tissue or region, the water protons of that region experience a significant relaxation increase, which can easily be detected in $T_{1}$-weighted

10 imaging sequences. For these reasons, contrast agents are important diagnostic tools used in more than $30 \%$ of all clinical MRI scans. However, as will be seen from the theory, the efficacy of a typical Gd(III) small chelate (such as, for example, gadodiamide) is quite limited, and a relatively large

15 local concentration of $\mathrm{CA}$ is required to obtain a significant contrast.

As a further complication, the design of all CA must cope with the possible toxicity of paramagnetic ions - notably Gd(III) among other lanthanides - and with the body excretion capabilities. Highly stable Gd(III) complexes are routinely used as CAs, yet not without insidious shortcomings. First, the gadolinium ion may coordinate regions of partial charge on proteins or macromolecular substrates and, as a consequence of such binding, the relaxivity of the CA can vary significantly due to the altered rotational and exchange dynamics. Second, the rapid tumbling of a small complex reduces the efficiency of the relaxation agent, as will be seen from the theory. To alleviate this problem, a variety of slow-tumbling water-soluble complexes with Gd(III) ions have been originally devised with the 0 aim of obtaining high water relaxivities at the lowest possible concentrations. Such macromolecular species include zeolites, micellar aggregates, polyamino acids, polysaccharides, dendrimers and, more recently, nanoparticles based either on lanthanide compounds or functionalised with lanthanide ion chelates. ${ }^{111}$

\subsection{Theoretical highlights}

The CA operating mechanism takes advantage of a phenomenon known as paramagnetic relaxation enhancement (PRE) of longitudinal and transverse relaxation rates. The interested can find a thorough treatment of PRE in books by Bertini $^{112}$ and Kowalewski, ${ }^{113}$ as well as in many technical reviews; to our scope, just the basics of the theory will be recalled in this section.

Solution-state NMR used by chemists mostly deals with diamagnetic species, i.e. species in which the pairing of the electron spins cancels out the electron magnetism. ${ }^{114}$ The strong magnetism of paramagnetic (and ferromagnetic) species is rather due to the presence of unpaired electron spins. In the presence of a magnetic field, the unpaired electron spins behave in a similar way to the nuclear spins, one major difference being that the electronic magnetic moment is about 650 times that of the proton. Just as the longitudinal and transverse relaxation of nuclear spins are driven by random fluctuations of the dipolar coupling between the nuclei, PRE is caused by fluctuations of the electron spin-nuclear spin interactions.

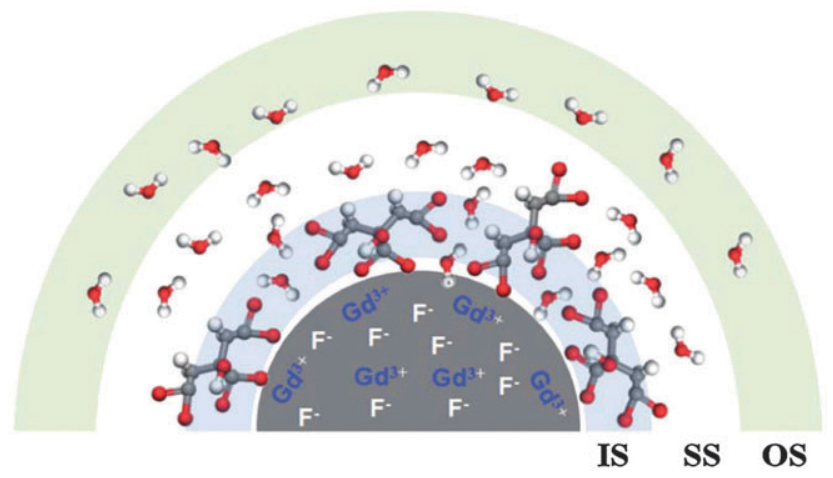

1

Fig. 15 Schematic representation of a $\mathrm{GdF}_{3}$ nanoparticle coated with citrate in water medium. IS = inner sphere; $\mathrm{SS}=$ second sphere; OS = outer sphere. Adapted from ref. 115 Copyright 2013 The Royal Society of Chemistry.

In solutions of paramagnetic ions or complexes, the ligands carrying nuclear spins (e.g. water) can reside in two types of environment, namely in the coordination sphere of the paramagnetic ion or in the bulk solution. PRE for each of these two scenarios is conveniently treated in different ways: "innersphere" relaxation refers to the relaxation enhancement of a solvent molecule directly coordinated to the paramagnetic ion, and "outer-sphere" relaxation refers to the relaxation enhancement of non-coordinated solvent molecules (namely, the bulk solvent). An intermediate situation, the "second-sphere" relaxation mechanism, generally refers to PRE of water not directly bound to the paramagnetic ion, but to other H-bonding groups in close proximity to the ion (e.g. carboxylates, see Fig. 15).

It is important to point out that the PRE effects discussed below can affect $T_{1}$ or $T_{2}$ (or even both) in distinct amounts, depending on the chemistry of the paramagnetic relaxing agent. In the following discussion, the Gd(III) ion in water will be taken as the paradigm of a $T_{1}$ relaxing agent: accordingly and for the sake of simplicity - only the theoretical formulations relative to $T_{1}$ will be explicitly mentioned. The analogous expressions for $T_{2}$ can be found in specialized books. ${ }^{112,113}$

\subsection{Inner-sphere relaxation}

For a dilute solution of paramagnetic species like Gd(III) in water, the inner-sphere contribution to PRE is given by the quantity

$$
T_{1 \mathrm{P}}{ }^{-1}=\frac{P_{\mathrm{M}}}{\tau_{\mathrm{M}}+T_{1 \mathrm{M}}}=R_{1 \mathrm{P}}
$$

where $P_{\mathrm{M}}$ is the mole fraction of bound water nuclei $\left(P_{\mathrm{M}}=[\mathrm{Gd}(\mathrm{III})] q / 55.6\right.$ with $q$ the number of binding sites in the complex $\left.^{111}\right), \tau_{M}$ is the lifetime of the water in the complex and $T_{1 \mathrm{M}}$ is the longitudinal relaxation time of the bound water protons. Note that $\tau_{M}$ is a limiting factor for the propagation of PRE from the paramagnetic site to the bulk water. For nonbinding paramagnetic complexes, the inner-sphere contribution is null by definition, whereas in the fast-exchange limit $\left(\tau_{\mathbf{M}}\right.$ $\left.\ll T_{1 \mathrm{M}}\right)$ the enhanced relaxivity depends on the relaxation time for the coordinated solvent molecules. 
The quantity $T_{1 \mathrm{M}}$ is made up of two contributions, namely a Fermi-contact (or scalar) one and a dipolar one. With some convenient approximations, $T_{1 \mathrm{M}}$ can be estimated by means of the Solomon-Bloembergen equations as

$$
\begin{gathered}
T_{1 \mathrm{M}}{ }^{-1}=\left(T_{1 \mathrm{M}}^{\mathrm{FC}}\right)^{-1}+\left(T_{1 \mathrm{M}}^{\mathrm{DD}}\right)^{-1} \\
\left(T_{1 \mathrm{M}}^{\mathrm{FC}}\right)^{-1}=\frac{2}{3} A_{\mathrm{FC}}{ }^{2} S(S+1) \frac{\tau_{\mathrm{e} 2}}{1+\left(\omega_{S}-\omega_{I}\right)^{2} \tau_{\mathrm{e} 2}{ }^{2}}
\end{gathered}
$$

$$
\left(T_{1 \mathrm{M}}^{\mathrm{DD}}\right)^{-1} \approx \frac{2}{15} \frac{K_{I S}{ }^{2}}{r_{I S}{ }^{6}} S(S+1)\left[\frac{7 \tau_{\mathrm{c} 2}}{1+\omega_{S}{ }^{2} \tau_{\mathrm{c} 2}{ }^{2}}+\frac{3 \tau_{\mathrm{c} 1}}{1+\omega_{I}{ }^{2} \tau_{\mathrm{c} 1}{ }^{2}}\right]
$$

In eqn (3a) the constant $A_{\mathrm{FC}}$ represents the scalar part of the Fermi contact interaction (i.e. the electron-nuclear hyperfine coupling constant), a quantity which is proportional to the density of "unpaired electron spin" at the site of the nucleus under investigation. The correlation time $\tau_{\mathrm{e}}=\left(\tau_{\mathrm{M}}{ }^{-1}+T_{2 \mathrm{e}}{ }^{-1}\right)^{-1}$ takes into account the exchange lifetime of the complex $\left(\tau_{\mathrm{M}}\right)$ and the electron transverse relaxation time $\left(T_{2 \mathrm{e}}\right)$. In eqn $(3 \mathrm{~b}) r_{I S}$ is the distance between the observed nucleus and the paramagnetic centre, where it can be defined. In addition, the above equations are formulated for a generic electron spin whose quantum number $S$ should be substituted by the spin-orbit $J$ quantum number in the case of lanthanides. The constant $K_{I S}$ is the (electron-nuclear) dipole-dipole coupling constant

$$
K_{I S}=-\frac{\mu_{0}}{4 \pi} \gamma_{I} \gamma_{S} \hbar
$$

$\gamma_{i}$ being the magnetogyric ratio of the nucleus $(i=I)$ or the electron $(i=S)$ and $\mu_{0}$ the permeability of vacuum. Again, the product $\gamma_{S} \hbar$ should be substituted with $g_{J} \mu_{\mathrm{B}}$ for the case of lanthanides, where $\mu_{\mathrm{B}}$ is the Bohr magneton.

It follows from the previous statements that the Fermicontact contribution vanishes "through bonds" (yet in a nonsystematic way), whereas the dipolar contribution rapidly vanishes "through space" as the distance $r_{I S}$ grows.

A prominent role in determining the relaxation properties is played by the correlation time quantities $\tau_{\mathrm{i}}$ : as a general rule, if more than one (uncorrelated) processes modulate a single interaction, the inverse of the resulting correlation time is a sum of the rates of the single processes responsible for the loss of correlation. For the dipolar part there exist two relevant correlation times, namely $\tau_{\mathrm{c} 1}$ and $\tau_{\mathrm{c} 2}$, defined as

$$
\tau_{\mathrm{cj}}{ }^{-1}=\tau_{\mathrm{R}}{ }^{-1}+\tau_{\mathrm{M}}{ }^{-1}+T_{j \mathrm{e}}{ }^{-1} ; j=1,2
$$

which incorporate the rotational correlation time $\tau_{\mathrm{c}}$ and the exchange lifetime of the complex together with the electron longitudinal $\left(T_{1 \mathrm{e}}\right)$ or transverse $\left(T_{2 \mathrm{e}}\right)$ relaxation times.

The ultimate goal in the design of highly efficient CAs is essentially related to shortening $T_{1 \mathrm{P}}$ as much as possible. Apart from increasing the number of binding sites $q$, a straightforward way to increase the relaxivity of $\mathrm{Gd}(\mathrm{III})$ complexes is to shorten $T_{1 \mathrm{M}}$. This goal can be achieved by increasing the rotational correlation time (see eqn (3b) and (5)), that is by increasing the size of the complex. The effect of this strategy is well illustrated in Fig. 16: the interaction between the complex

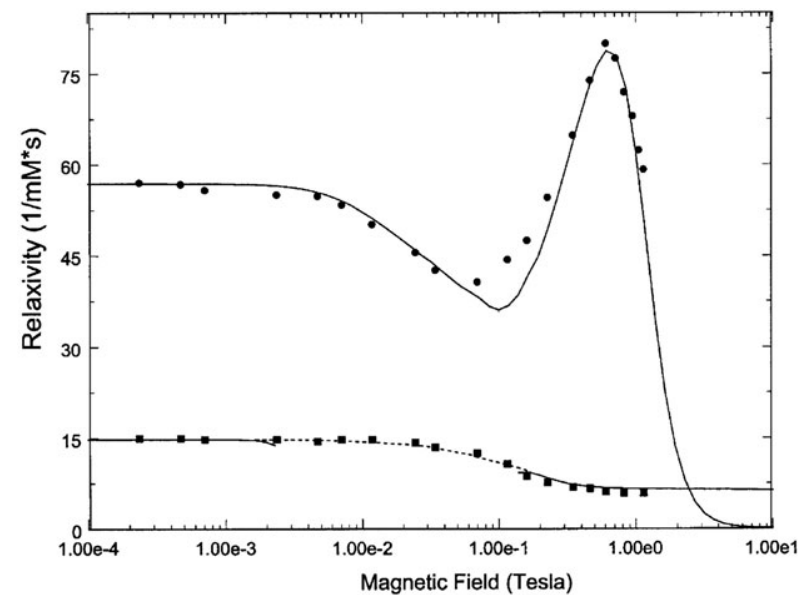

Fig. 16 Experimental and calculated nuclear magnetic relaxation dispersion (NMRD) profiles for Gd-EDTA ${ }^{-}$in aqueous solution in the presence (solid circles) and absence (solid squares) of the bovine serum albumin. Adapted from ref. 116 Copyright 2003 Elsevier Ltd.

Gd-EDTA $^{-}$and bovine serum albumin clearly raises the relaxivity and modifies the dispersion profile.

\subsection{Outer-sphere relaxation}

As the exchange lifetime $\tau_{M}$ becomes increasingly shorter, a limit situation is reached where a binding site no longer exists and the paramagnet-ligand interaction is modulated only by random encounters between the molecules, under diffusion control. In other words, the solvent (water) molecules can also experience PRE even in the absence of coordination to the paramagnetic species. This second mechanism is generally called "outer-sphere" relaxation and, differently from the inner-sphere intramolecular mechanism, it is driven by the stochastic variation of the intermolecular nuclear spin-electron spin distance. In fact, pure "outer-sphere" relaxation arises just from the modulation of the electron-nuclear dipolar coupling by translational diffusion of water molecules around the Gd(III) complex. In between the "inner-sphere" and "outer-sphere" mechanisms, a "second coordination" (or secondary-sphere) effect has sometimes been invoked, yet hardly quantified. ${ }^{117,118}$ Within this picture, contact shifts no longer exist, and the dipolar shifts average to zero (if the approach to the paramagnetic ion can occur in every direction). ${ }^{112}$ As compared to eqn (1), the equations describing $T_{1}$ for the outer-sphere relaxation are quite different, although a general factorization into a constant term and a dynamic term can still be identified. The characteristic time for the modulation of the interaction energy becomes the diffusional correlation time $\tau_{\mathrm{D}}$,

$$
\tau_{\mathrm{D}}=\frac{d^{2}}{D_{\mathrm{M}}+D_{\mathrm{L}}}
$$

where $D_{\mathrm{M}}$ and $D_{\mathrm{L}}$ are the diffusion coefficients of the Gd(III)containing and ligand-containing (water) molecules, and $d$ is the distance of closest approach between the Gd(III) and the water molecules. Note that, for very large (e.g. nanosized) 
1 complexes, $\left(D_{\mathrm{M}}+D_{\mathrm{L}}\right) \sim D_{\mathrm{L}}$ meaning that only the ligand (water) diffusion coefficient can influence $\tau_{\mathrm{D}}$.

Within the outer-sphere relaxation mechanism, two limiting situations apply, depending on whether the electronic relaxation time is shorter or longer than the diffusional correlation time.

In the case of fast electron relaxation, the solvent appears frozen on the timescale of the electron relaxation process. The outer-sphere contribution can be thus evaluated by integrating the dipolar part of the Solomon-Bloembergen eqn (3b) over the distance range between $d$ and infinity, while noting that $\tau_{\mathrm{c} j}=T_{j \mathrm{e}}$ from eqn (5). In doing this, the following equation results:

$$
\begin{aligned}
T_{1 \mathrm{M}}{ }^{-1}= & \frac{2}{15} 1000 N_{\mathrm{A}}[\mathrm{M}] \frac{4 \pi}{3} \frac{K_{I S}{ }^{2}}{d^{3}} S(S+1) \\
& \times\left[\frac{7 T_{2 \mathrm{e}}}{1+\omega_{S}^{2} T_{2 \mathrm{e}}^{2}}+\frac{3 T_{1 \mathrm{e}}}{1+\omega_{I}^{2} T_{1 \mathrm{e}}{ }^{2}}\right]
\end{aligned}
$$

where $1000 N_{\mathrm{A}}[\mathrm{M}]$ is the concentration of the molecule bearing the paramagnetic centre in mol m${ }^{-3}, N_{\mathrm{A}}$ being the Avogadro number. In this case, the relevant parameters are just the concentration and the longitudinal and transverse electronic relaxation times of the paramagnetic species. Also, different from the inner-sphere mechanism, the paramagnetic effects for the outer sphere mechanism are not reduced by the mole fraction $P_{\mathrm{M}}$ of bound solvent molecules.

In contrast to the previous case, in the diffusion-controlled regime the dipolar interaction is modulated only by the relative diffusion of the paramagnetic centre and the solvent spins. Eqn (7) thus can be rewritten as

$$
\begin{aligned}
T_{1 \mathrm{M}}{ }^{-1}= & \frac{8}{135} 1000 N_{\mathrm{A}}[\mathrm{M}] \frac{4 \pi}{3} \frac{K_{I S}{ }^{2}}{d\left(D_{\mathrm{M}}+D_{\mathrm{L}}\right)} S(S+1) \\
& \times\left[7 \mathcal{J}\left(\omega_{S}\right)+3 \mathcal{J}\left(\omega_{I}\right)\right]
\end{aligned}
$$

where the quantities $\mathcal{J}\left(\omega_{i}\right)$ are the spectral densities for the modulation of the distance $r$ by (uniform) translational diffusion. As has been pointed out in different contexts, ${ }^{119-121}$ such spectral densities scale as the inverse of the distance of closest approach $d$, rather than as the inverse sixth power of $r$ (eqn (3b)), allowing PRE to reach long-range water spins.

Generally, for monoaqua ( $q=1)$, low molecular weight Gd(III) complexes at high fields, the outer-sphere contribution can account for nearly $50 \%$ of the relaxivity, whereas its contribution drops in macromolecular systems in the approximate frequency range $10-60 \mathrm{MHz} .{ }^{111}$

\subsection{Curie spin relaxation}

The static magnetic moment $\langle\mu\rangle$ of a paramagnetic molecule interacts with the nuclear spins and introduces another relaxation mechanism. This contribution is important for large paramagnetic molecules with rapid electron spin relaxation, and can in certain situations prevail over the Solomon-like terms. Curie spin relaxation is modulated by reorientation and exchange dynamics of the paramagnetic complex, but not by its electron relaxation. In addition, since the magnetic moment depends on the square of the external magnetic field, Curie spin relaxation increases with the square of the nuclear Larmor frequency $\omega_{I}$, according to the equation

$$
T_{1 \mathrm{M}}{ }^{-1}=\frac{2}{5}\left(\frac{\mu_{0}}{4 \pi}\right)^{2} \frac{\omega_{I}^{2} g_{\mathrm{e}}{ }^{4} \mu_{\mathrm{B}}{ }^{4}}{\left(3 k_{\mathrm{B}} T\right)^{2} r_{I S}{ }^{6}} S^{2}(S+1)^{2}\left[\frac{3 \tau_{\mathrm{r}}}{1+\omega_{I}{ }^{2} \tau_{\mathrm{r}}{ }^{2}}\right]
$$

where the constants and parameters have been already introduced in eqn (3) and (4), except for the Boltzmann constant $k_{\mathrm{B}}$. As seen for the previous equations, the spin-orbit $J$ quantum number should replace the quantum number $S$ in the case of lanthanides. The quantity $S_{\mathrm{C}}=\left\langle S_{z}\right\rangle=-g_{\mathrm{e}} \mu_{\mathrm{B}} S(S+1) B_{0} /\left(3 k_{\mathrm{B}} T\right)$ appearing in eqn (9) is the magnitude of the "Curie spin", namely the thermally averaged amount of spin $S$ aligned along the external magnetic field $B_{0}$. In this respect, the Curie spin $S_{\mathrm{C}}$, the magnetic moment $\langle\mu\rangle$ and the molar susceptibility $\chi_{\mathrm{M}}$ represent alternate viewpoints on the same phenomenon, being $\langle\mu\rangle=-g_{\mathrm{e}} \mu_{\mathrm{B}} S_{\mathrm{C}}$ and $\chi_{\mathrm{M}}=\mu_{\mathrm{B} 0} N_{\mathrm{A}}\langle\mu\rangle / B_{0}$.

At high field strengths with slowly rotating molecules the Curie spin relaxation mechanism may become important, but it is generally negligible at the low fields used in MRI (typically up to $1.5 \mathrm{~T}$, about $60 \mathrm{MHz}$ proton Larmor frequency). ${ }^{117}$

\subsection{Lanthanide-based nanoparticles as MRI contrast agents}

The use of nanomaterials and nanoparticles as contrast agents has attracted much attention in the last few years, and this subject has been quite extensively covered by recent reviews. ${ }^{111,122-124}$ Examples of lanthanide-based nanosystems include surface-coated lanthanide oxide nanoparticles ${ }^{125,126}$ such as $\mathrm{Gd}_{2} \mathrm{O}_{3}$ (also doped to add luminescent or fluorescent upconverting properties ${ }^{43}$ ), where the coating is typically glucuronic acid, $^{7}$ lactobionic acid, ${ }^{127}$ polyethylene glycol $(\mathrm{PEG})^{128,129}$ to ensure biocompatibility and avoid precipitation. Ultrasmall $\mathrm{Gd}_{2} \mathrm{O}_{3}$ nanoparticles with particle diameters of about $1 \mathrm{~nm}$ exhibit a large $R_{1}$ relaxivity with a maximum between 1 and $2.5 \mathrm{~nm}$ diameter.

Along the same line, dextran coated paramagnetic gadolinium phosphate $\left(\mathrm{GdPO}_{4}\right)$ nanoparticles have been proposed for MRI tumor imaging, relying on their tens of nanometre size for accumulation into tumor cells, and on the dextran coating to prevent the rapid elimination from the blood stream. ${ }^{130}$

Layered gadolinium hydroxychloride nanoparticles $\left[\mathrm{Gd}_{2}(\mathrm{OH})_{5}\left(\mathrm{H}_{2} \mathrm{O}\right)_{x}\right] \mathrm{Cl}$ have also been proposed as MRI contrast agents: interestingly, such systems can be dispersed to form a stable colloidal nanosheet in aqueous medium. ${ }^{125}$

Gadolinium fluorides (i.e. $\mathrm{GdF}_{3}$ and $\mathrm{NaGdF}_{4}$ ) have also attracted growing interest due to their chemical versatility and high relaxivity values. ${ }^{131}$ With respect to other inorganic NPs, these systems have the ability to quickly and reversibly exchange their cations by surface exposure to another lanthanide ion: indeed, this peculiar feature opens up the way to a straightforward synthesis of multimodal imaging NPs bearing, for instance, an up-conversion core and a paramagnetic shell. ${ }^{132}$ A study reported by van Veggel and co-workers ${ }^{133}$ showed that the rate of increase in ionic relaxivity with decreasing NP size closely parallels the rate of increase in the NP surface-to-volume ratio, thus giving indication that the surface

1

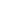


1 Gd ions are the major contributors to the relaxivity enhancement. Furthermore, the surface Gd ions on a larger NP have been shown to affect the relaxivity more strongly than those on a smaller NP, which is consistent with the theory of innersphere relaxation.

Botta and co-workers ${ }^{115}$ have provided a thorough investigation into the structure and dynamics of the hydration shells of citrate-coated $\mathrm{GdF}_{3}$ nanoparticles (Fig. 15). In this study, different citrate: $\mathrm{Gd}(\mathrm{III})$ molar ratios were used to investigate the effect of the organic shell on the relaxometric properties. The resulting NPs were thus investigated by nuclear magnetic relaxation dispersion, with the aim of understanding the operating PRE mechanisms and assessing their relative contributions. The longitudinal relaxivity $\left(1 / T_{1}\right)$ for these NPs is found to significantly lower than the typical values of $\mathrm{Gd}$-chelates conjugated to macromolecules or incorporated into nanosized systems (micelles, liposomes, silica NPs). The rationale behind this evidence lies in the fact that, in "chelated" macromolecular systems, all paramagnetic ions are accessible to the solvent and contribute to the relaxivity enhancement, mainly because of the reduced molecular tumbling. By means of NMRD measurements complemented with variable-temperature ${ }^{17} \mathrm{O}$ experiments the same authors conclude that, for $\mathrm{GdF}_{3}$ nanoparticles, $\mathrm{Gd}^{3+}$ surface ions are coordinated to citrate anions and to two inner-sphere water molecules which extensively contribute to $R_{1 \mathrm{p}}$. The second-sphere mechanism is also important in this case, and corresponds to the presence of four water molecules per metal ion on the surface, whereas the outersphere contribution is found to be negligible.

Another interesting system for the investigation of the PRE induced by lanthanide NPs is represented by the core@shell $\mathrm{Gd}^{3+}$ doped upconverting nanoparticles (UCNP) proposed by Bu and Shi and co-workers. ${ }^{134}$ In the case of silica-shielded NPs (Fig. 17), the

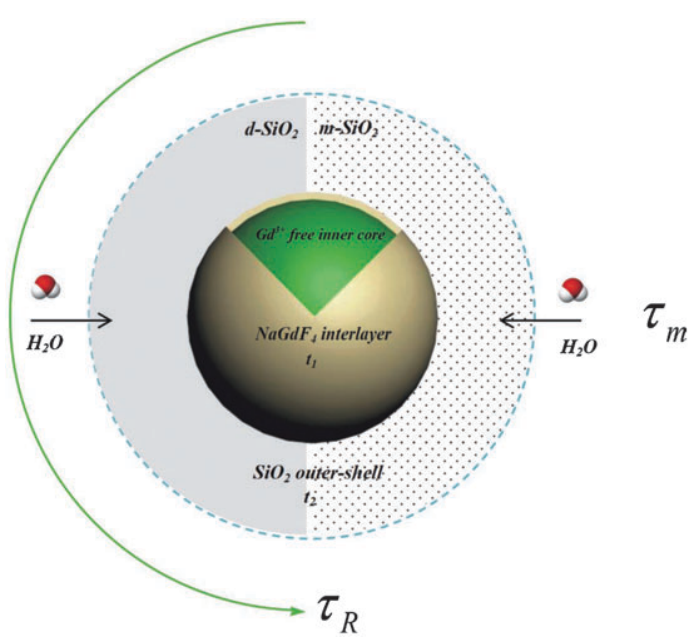

Fig. 17 Sketch of a silica protected, water soluble coreaNaGdF 4 nanoparticle. The interlayer $\mathrm{NaGdF}_{4}$ is the PRE active layer ( $t_{1}=$ thickness), which contains thousands of surface $\mathrm{Gd}^{3+}$ ions as payload. Shell of tunable thickness $\left(t_{2}\right)$ dense (d-) and mesoporous (m-) silica covers the single particle to create difference in porosity and particle size, which could potentially alter the coordinated number of water molecules. Adapted from ref. 134 Copyright 2013 Wiley-VCH Verlag GmbH \& Co. KGaA, Weinheim.

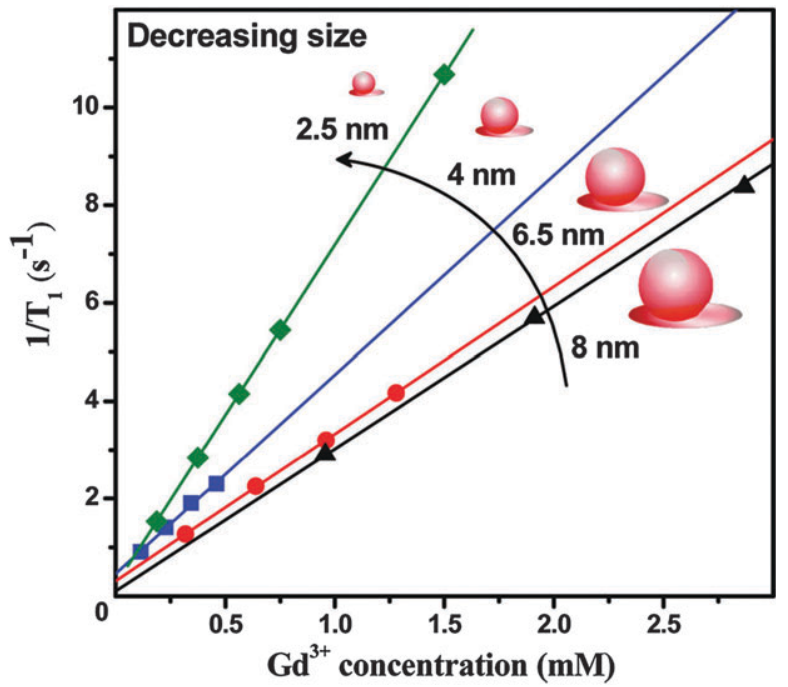

1

Fig. 18 Longitudinal relaxivity $\left(1 / T_{1}\right)$ of water protons per $\mathrm{Gd}(\mathrm{II})$ ion for $\mathrm{NaGdF}_{4} \mathrm{NPS}$ of different sizes in water (1.5 T). Surface-to-volume ratio S/V $=3 / r$ with $r$ the radius of the nanoparticle. Adapted from ref. 133 Copyright 2011 American Chemical Society.

inner-sphere mechanism is hampered by the slow diffusion of water through the silica shell, and the longitudinal relaxivity enhancement of water protons occurs mainly via the outersphere mechanism. It is worth remarking that, in macromolecular systems with chelate paramagnetic ions, the efficiency of PRE can be controlled by varying the size of the macromolecule (that is, $\tau_{\mathrm{c}}$ ) or the chemistry of the chelating groups (and hence $\tau_{\mathrm{M}}$ ). In lanthanide-based nanosystems, the lanthanide ions on the surface are the major contributors to the PRE of water protons, while the core ions do not provide a significant contribution to the relaxivity. ${ }^{135}$ PRE in these systems is thus driven by the surface-to-volume ratio $(S / V$, see Fig. 33$)$ and by a careful choice of the coating layer, which can possibly endow secondary sphere relaxation and control the water access to the NP surface.

However, the rotational correlation time $\tau_{\mathrm{c}}$ increases with the NP size, contrasting the $S / V$ effect and making the surface ions on larger NPs much more relaxation-efficient than those on smaller NPs. Clearly, the complex balance among all these effects is best assessed through the experimental evidence, as testified by the great amount of synthesis and characterization efforts that has been recently invested in these nanoprobes (Fig. 18).

Gadolinium-based NPs doped with fluorescent lanthanide elements have also attracted attention for their ability to act as MRI and fluorescence imaging agents. In principle, the combination of fluorescent bioimaging with MRI capabilities is appealing because the greater sensitivity and resolution of fluorescence imaging may complement MRI data. Examples of such systems include PEGylated $\mathrm{Gd}_{2} \mathrm{O}_{3}: \mathrm{Tb}^{3+} \mathrm{NPs}^{136}$ and mixed $\mathrm{Eu}_{0.2} \mathrm{Gd}_{0.8} \mathrm{PO}_{4} \cdot \mathrm{H}_{2} \mathrm{O}$ NPs. ${ }^{137} \mathrm{GdF}_{3}$ NPs doped with $\mathrm{Er}^{3+} /$ $\mathrm{Yb}^{3+}$ and $\mathrm{Tm}^{3+} / \mathrm{Yb}^{3+}$ have also been proposed as bimodal probes for both in vitro and in vivo optical and MRI, the latter technique taking advantage from the high $R_{2}$ relaxivity found 
1 for these hybrid NPs. ${ }^{138}$ Unfortunately, one of the general limitations of Gd-doped magnetic/upconversion fluorescent probes is their relatively low longitudinal relaxivity $R_{1}$, which can prevent their use in living systems. For the same UCNPs, a number of reports have shown that a fluorescence enhancement of lanthanide ions in the lattice can be obtained by introducing a coating layer that shields the surface emission ions and reduces the surface defects. As an example, a $\mathrm{NaYF}_{4}$ matrix can shield $\mathrm{Er}^{3+}$ ions from the surroundings and enhance

10 their fluorescent intensity as compared to those at the crystal surface, but the same matrix also isolates $\mathrm{Gd}^{3+}$ ions from the water molecules, thus reducing $R_{1}$. In practice, for such nanoprobes, deep ( $>4 \mathrm{~nm}$ ) Gd ${ }^{3+}$ ions do not contribute significantly to the longitudinal relaxivity. These observations have inspired the synthesis of a highly efficient nanosystem for bimodal $T_{1}$ MRI and upconversion fluorescent imaging. ${ }^{139}$ In such a way, Gd-doped UCNPs were designed with a thin $\mathrm{NaGdF}_{4}$ layer deposited on Gd-free $\mathrm{NaYF}_{4}: \mathrm{Er} / \mathrm{Yb}$ cores, achieving a $R_{1}$ value of $6.18 \mathrm{mM}^{-1} \mathrm{~s}^{-1}$.

Gadolinium oxysulfide NPs doped with other lanthanides $\left(\mathrm{Eu}^{3+}, \mathrm{Er}^{3+}, \mathrm{Yb}^{3+}\right)$ have been suggested as multimodal platforms for MRI, X-ray and photoluminescence imaging. ${ }^{140} \mathrm{Gd}_{2} \mathrm{O}_{2}$ $\mathrm{S}: \mathrm{Eu}^{3+}$ NPs show a strong transverse relaxivity and a strong $\mathrm{X}$-ray absorption, allowing their use as contrast agents for $T_{2}$-weighted MRI and X-ray tomography. Similarly, upconverting $\mathrm{Gd}_{2} \mathrm{O}_{2} \mathrm{~S}$ :Er; $\mathrm{Yb}$ NPs can be used both for in vivo deep fluorescence imaging and for MRI. Core-shell nanoparticles with $\mathrm{NaLuF}_{4}: \mathrm{Yb}^{3+}, \mathrm{Tm}^{3+}$ as the core and $\mathrm{SiO}_{2}$ as the shell layer, complemented with the complex Gd-DTPA as the surface ligand, have also been designed for trimodal NIR-to-NIR upconversion, MRI and X-ray tomography imaging. ${ }^{141}$

Bio-functionalized NPs with a core-shell structure have also been prepared for MRI and fluorescence imaging. These systems feature a Prussian blue core containing interstitial gadolinium and a biofunctional shell of fluorescent avidin and biotinylated antibodies that enable molecular targeting of eosinophilic cells. ${ }^{142}$

\subsection{Comparison with other MRI contrast agents based on Gd(III) complexes}

Among the many parameters appearing in the equations related to PRE, only a few are actually in control of the synthetic chemist, namely $\tau_{\mathrm{M}}$ (related to the exchange and to the chemistry of the chelating molecules) and $\tau_{\mathrm{c}}$ (related to the size of the decrease in the correlation time of a lanthanide complex can lead to a significant improvement in the relaxivity, and much effort has been devoted to the conjugation of Gd(III) complexes with macromolecules or nanoscopic objects via bifunctional exchange $\tau_{\mathrm{M}} \ll T_{1 \mathrm{M}}$ is easily met for small complexes, the situation changes significantly for macromolecular conjugates, where the fast exchange condition is much more stringent and requires $\tau_{M}$ to be in the order of tens of nanoseconds. ${ }^{111}$ This regime is hardly met by lanthanide-DOTA or lanthanide-DTPA macromolecular conjugates, for which an intermediate- or slow-exchange regime typically applies $\left(\tau_{\mathrm{M}} \geq T_{1 \mathrm{M}}\right)$. Indeed, proper chemical tuning may recover the fast exchange regime, but the requirement of clinically approved complexes of exceptional stability severely limits the design of new ligands for in vivo applications.

Amplification of an otherwise modest ionic relaxivity can be achieved through scaffolds that accommodate a large number of paramagnetic complexes on a single macromolecule. In this context dendrimers, monolayer-protected nanoparticles and virus capsids have provided perhaps the best results in terms of relaxivity gains (see Table 2). Liposomes have also been prepared by the self-assembly of amphiphilic units bearing chelate Gd ions. Albeit of limited "per unit" efficiency, the appeal of liposomes for in vivo imaging mainly stems from their high biocompatibility and easy manipulation of their physicochemical properties. Furthermore, in the wide list of nanoscopic MRI contrast agents, a distinctive role is played by super paramagnetic iron oxide nanoparticles (SPIOs), whose clinical use (e.g. Combidex) relies on their ability to strongly enhance $T_{2}$-weighted images, particularly in liver, spleen, and bone marrow.

As compared to other macromolecular contrast agents, it can be seen from Table 2 that lanthanide-based nanoparticles have a relatively low per unit relaxivity, which is partly recovered by increasing the number of paramagnetic centres loaded. Indeed, the capability to act as multimodal imaging agents still represents an unmatched and most attracting characteristic of this class of compounds.

Despite the established use of lanthanide-based drugs in clinical MRI, the biotoxicity of their nanoscopic analogues is not extensively studied and still little understood. ${ }^{143}$ In general, macromolecular conjugates tend to have a much longer dwell time in the body and a more difficult elimination, their toxicity varies with the type, size and surface functional groups. SPIO nanoparticles, the only class of NPs clinically used as MRI contrast agents, seem to have a high in vitro toxicity, which can be however attenuated by proper coating (namely with dextran). Observed toxic effects range from cell death to inflammatory reactions, and from particle agglomeration to alterations in neurobehavior. Most of the nanoparticles listed in Table 2 show prolonged tissue retention and contain heavy metals, something that is highly concerning from a clinical perspective. The long-term in vivo toxicity is generally evaluated in rats or mice by weight measurement, behaviour observation, histology and haematology analysis: yet, the toxicity evaluation protocols still lack uniformity as far as the physicochemical properties and the nanomaterials' applications are concerned. ${ }^{55}$

\section{Multi-modal NPs for other imaging techniques}

Lanthanide-based NPs can be conveniently used also for imaging techniques other than MRI and OI, like CT, PET, and SPECT. In the majority of cases, however, this occurs when dual- or tri-modal nanoprobes are designed, typically 


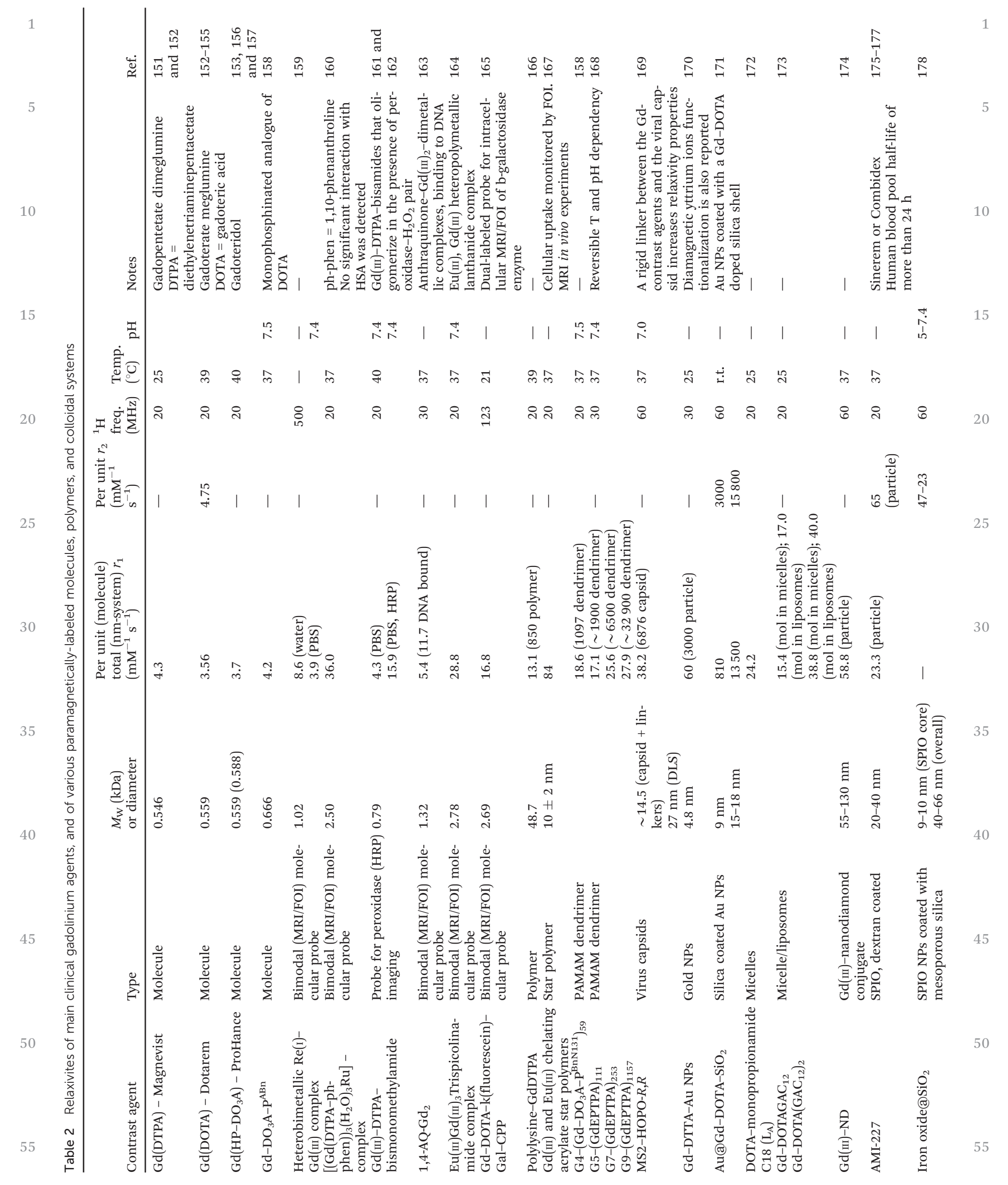




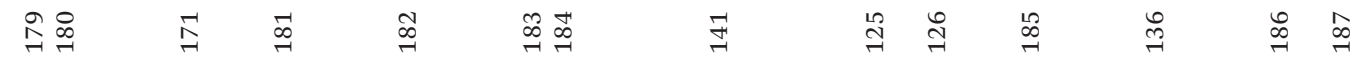

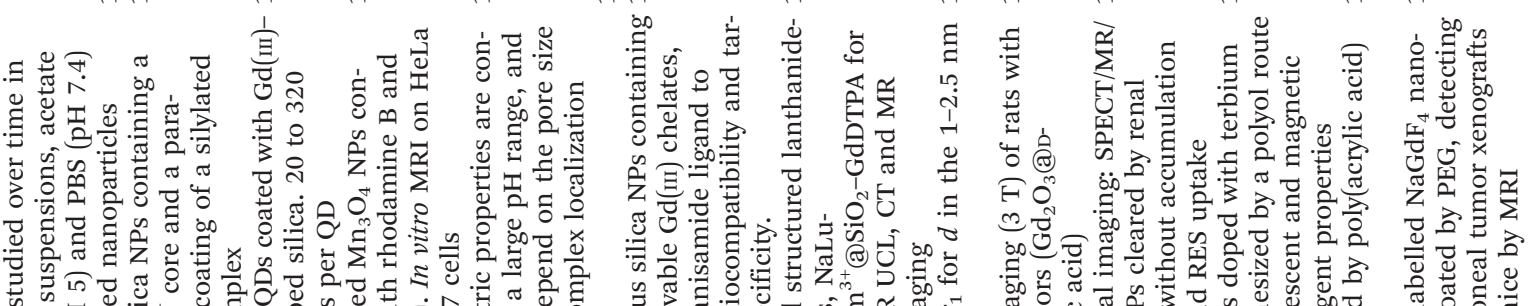

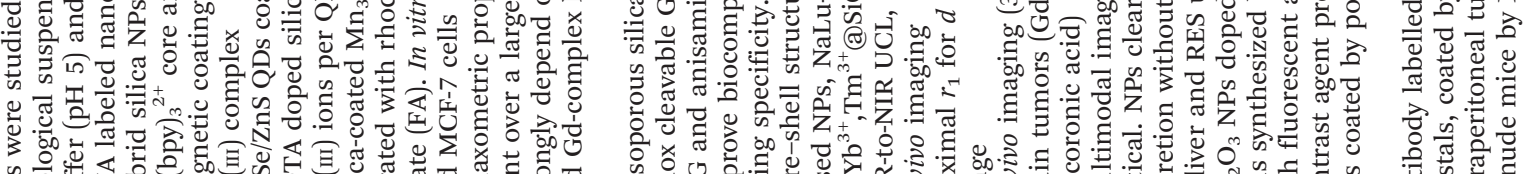

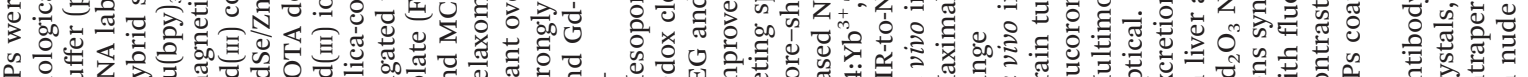

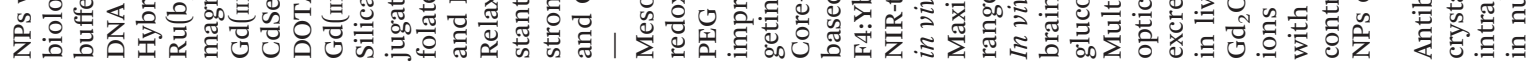
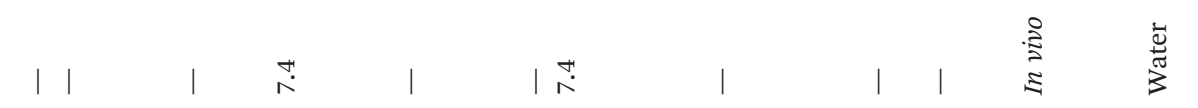

苛

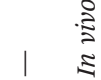

$$
\text { के के के के के }
$$

육 \&

กิ

l 1

20

$\stackrel{\Im}{\Im}$
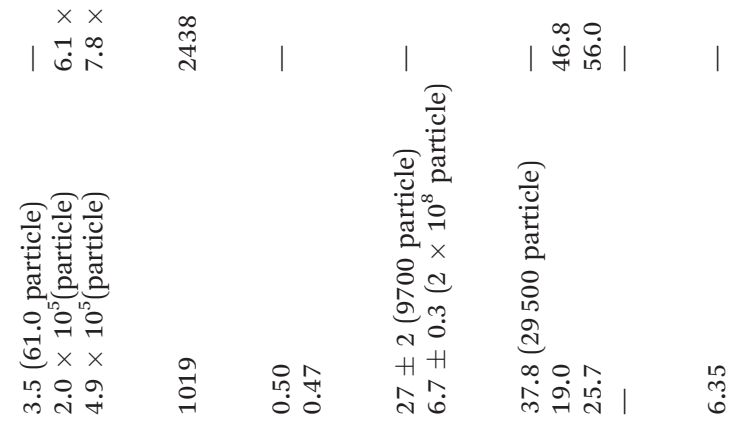

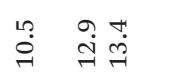

$\frac{4}{n}$

เูล

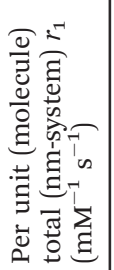

mi

A

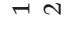
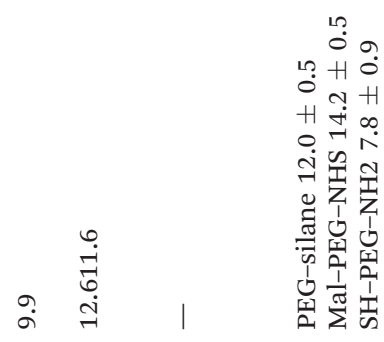

节宰|

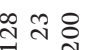

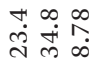

苛

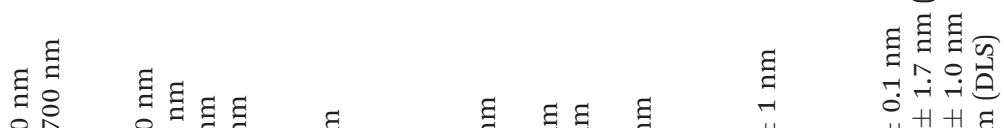

H + H

ஸे

云全年

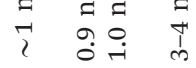
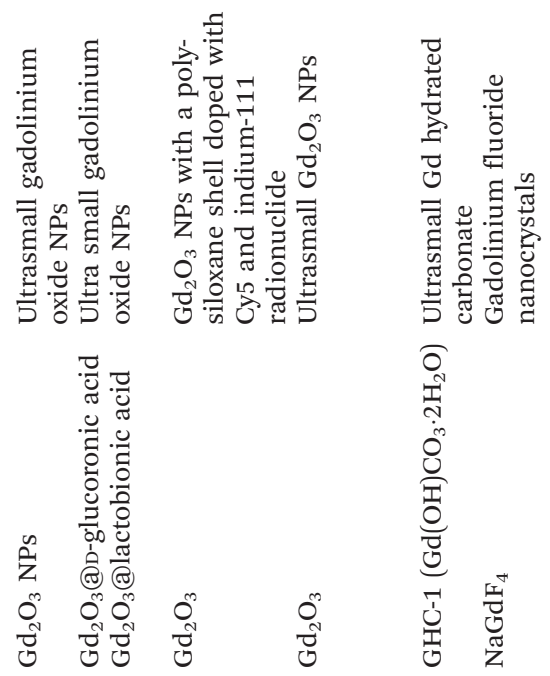


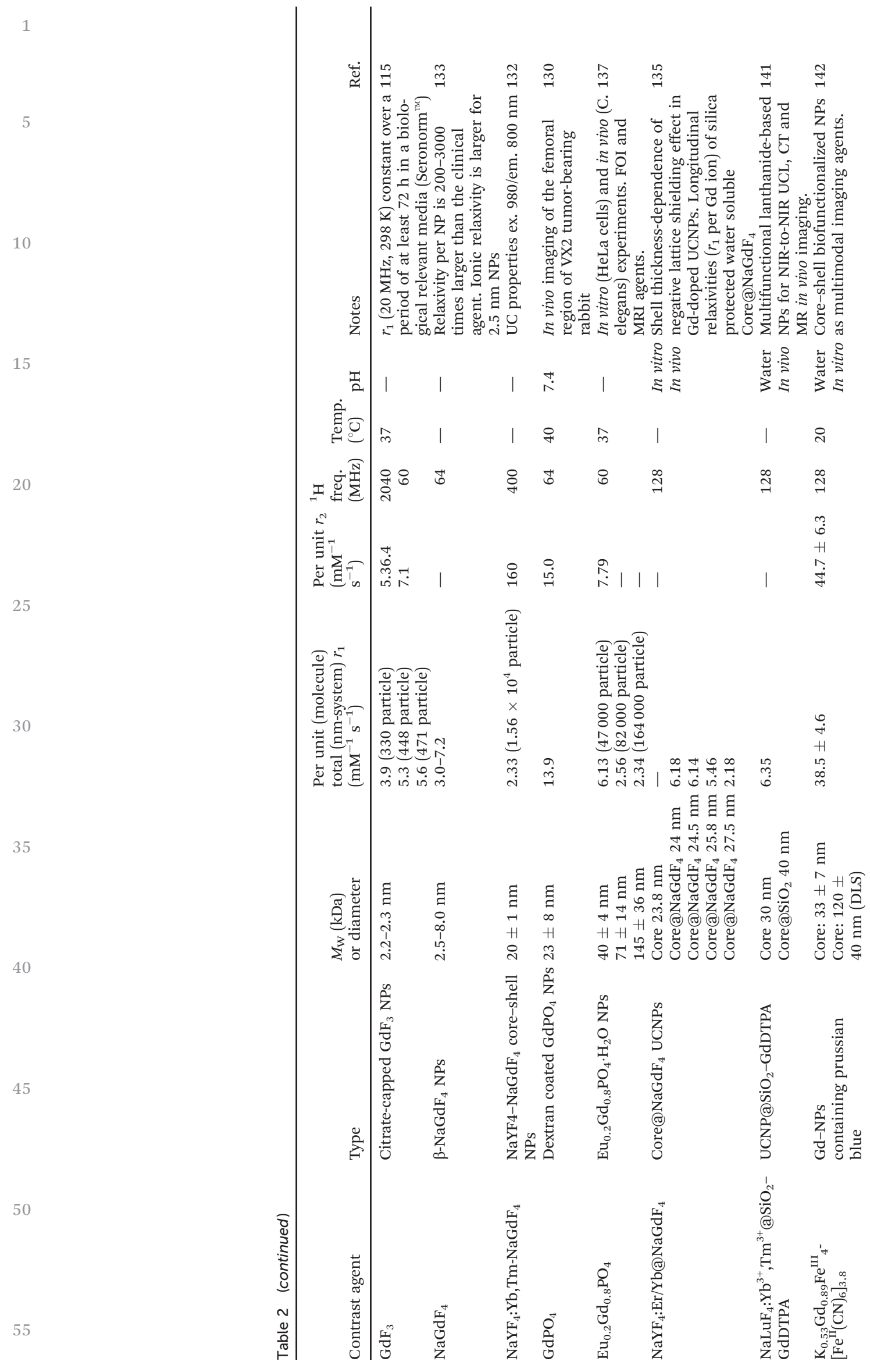


1 combining UPCNs for OI with one of the other imaging techniques. To our opinion, it is important to remind at this point that, as already discussed, the preparation of multimodal probes can help us to overcome some of the limitations characteristic of a single modality; it is however often underestimated the problem associated with the different sensitivity offered by the various imaging techniques, which could hamper the use of the same probe under the same conditions (e.g. concentration) with two different modalities.

\subsection{Nuclear medical imaging}

As already mentioned, from one hand both PET and SPECT, being based on radioisotopes, can benefit from a very high 15 sensitivity and unlimited tissue penetration; on the other hand, the same decay causes health concern, disposal related problems, and possible limitations to synthetic strategies. This last problem is particularly important in nanomedicine: the probe, in fact, has to be prepared and purified in a time shorter, or at

20 least comparable, to the half-life of the nuclide. This severely limits the number of possible solutions, especially when, instead of rather simple molecules as ${ }^{18}$ FDG, a multicomponent nanostructure has to be prepared. For example, the most used radionuclide for clinical PET is ${ }^{18} \mathrm{~F}$, which has a half-life time of circa 110 minutes: a very rapid conjugation of ${ }^{18} \mathrm{~F}$ into the NPs and a very high reaction yield are thus required in this case. To date, the only efficient strategy for the preparation of rare earth NPs for PET imaging has been proposed by the group of F. Y. Li and is based on the strong binding between fluoride anions and rare-earth cations. ${ }^{144-146}$ In particular, a water solution containing ${ }^{18} \mathrm{~F}$ is typically added to a water dispersion of NPs; after a rather short (1-10 minutes) incubation, the NPs are purified by centrifugation. Since this strategy is simple and general, it has been used with NPs with different cores (such as

$35 \mathrm{Y}_{2} \mathrm{O}_{3}, \mathrm{NaYF}_{4}, \mathrm{Y}(\mathrm{OH})_{3}, \mathrm{Gd}(\mathrm{OH})_{3}$, eventually co-doped for obtaining UC material) and capped with various agents (citrate, Pluronic F127 or oleic acid/ $\alpha$-CD). In a first example, with this strategy Li and coworkers ${ }^{145}$ synthesized ${ }^{18} \mathrm{~F}$-labeled hydrophilic citrate-capped $\mathrm{NaY}_{0.2} \mathrm{Gd}_{0.6} \mathrm{Yb}_{0.18} \mathrm{Er}_{0.02} \mathrm{~F}_{4}$ probes made for 40 PET, MRI, and UCL imaging. In particular, using a smallanimal micro-PET scanner, 15 min after injection on anesthetized mice an intense signal was detected almost exclusively in the liver and spleen (Fig. 19). This was in agreement with the magnetic resonance images obtained with the same set of NPs that, however, are characterized by a low contrast. No evidence of clearance from these organs was observed after two hours.

In another example by the same group, ${ }^{146} \mathrm{NaYF}_{4}: \mathrm{Yb}, \mathrm{Tm}$ UCNPs, capped with PEG and having a $20 \mathrm{~nm}$ diameter, were derivatized with ${ }^{18} \mathrm{~F}^{-}$, and applied for lymph node monitoring.

50 An intense signal could be detected in the left sentinel lymph node even 3 min after subcutaneous injection of the NPs into the left paw of a nude mice; dynamic observation indicated in particular a dramatic increase of the signal between 0 and 30 min after injection, followed by a plateau. It is interesting to note that probes with dimensions ranging from 20 to $100 \mathrm{~nm}$ are of great interest for sentinel lymph node mapping, ${ }^{147}$ so

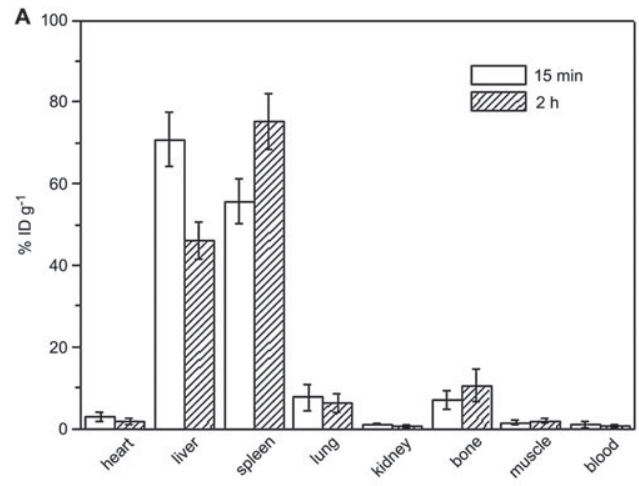

1

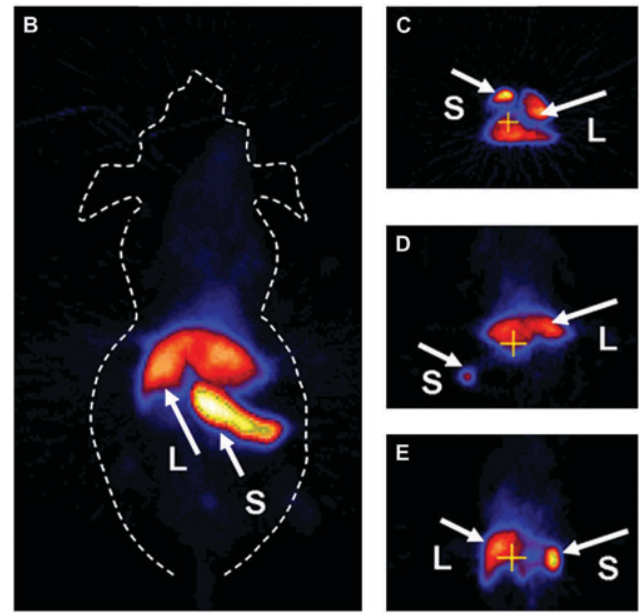

Fig. 19 (A) Biodistribution of $\sim 148 \mathrm{kBq}{ }^{18} \mathrm{~F}$-labeled-cit-NPs at $15 \mathrm{~min}$ and $2 \mathrm{~h}$ post-injection $(n=5)$. In vivo micro-PET images acquired at $15 \mathrm{~min}$ after intravenous injection of $\sim 5.9 \mathrm{MBq}{ }^{18} \mathrm{~F}$-cit-NPs. (B) Whole-body twodimensional projection, (C) transversal, (D) coronal, and (E) sagittal images are shown respectively. The arrows inset point to the liver $(\mathrm{L})$ and spleen (S). Adapted from ref. 145 Copyright 2011 Elsevier Ltd.

that the use of NPs is particularly promising for this crucial application.

The luminescence properties of these NPs (two distinct bands at 475 and $800 \mathrm{~nm}$ upon $980 \mathrm{~nm}$ CW excitation) were conveniently used to verify their distributions within organs in mice. Analogous results were also obtained in vivo, after improving the water solubility of OA capped lanthanidedoped nanocrystals via supramolecular alkyl chain/ $\alpha$ cyclodextrin $(\alpha-C D)$ host-guest association. This approach resulted independent from the nanocrystal size, shape and core composition, and was applied by $\mathrm{Li}$ and co-workers to $\mathrm{NaYF}_{4}: 20 \mathrm{~mol} \% \mathrm{Yb}, 2 \mathrm{~mol} \% \mathrm{Er}$ NPs obtained by thermal decomposition. The final multimodal system was suitable for cell labelling in vivo. ${ }^{144}$ These results clearly indicate that the formation of a supramolecular adduct with $\alpha$-CD can be widely applied in improving the water-solubility of UCNPs. The same strategy has also been used for preparing tri-modal (NMR, UCL, and PET) NPs by Li and coworkers, in this case as the final step of the synthetic procedure. ${ }^{148}$ They started from $\mathrm{NaYF}_{4}: \mathrm{Yb}, \mathrm{Er}$ oleate capped nanoparticles obtained by solvothermal synthesis, to develop multifunctional up-conversion nanoparticles $\left(\mathrm{NaYF}_{4}: \mathrm{Yb}, \mathrm{Er}\right)$, combining magnetic $\left(\mathrm{Gd}^{3+}\right)$, PET $\left({ }^{18} \mathrm{~F}\right)$, and 
1 targeted recognition (folic acid) properties. In particular $\mathrm{Gd}^{3+}$ was introduced on the surface of the nanocrystals by cation exchange with $\mathrm{Y}^{3+}$ ions, while ${ }^{18} \mathrm{~F}$ was introduced for PET imaging by interaction with the rare-earth ions.

In particular, surface $\mathrm{Gd}^{3+}$ ions obtained in this way have the advantage to present strong $T_{1}$ enhancement as already discussed. The incubation with ${ }^{18} \mathrm{~F}^{-}$anions followed the surface coverage with $\mathrm{OA}$, aminocaproic acid and folic acid, used to confer water solubility and target ability toward cancer cells.

10 The approach described so far has however two important limitations. The first one is intrinsic to the chosen radionuclide: the short half-life of ${ }^{18} \mathrm{~F}$ does not allow investigations for a time longer than circa $2 \mathrm{~h}$.

Moreover, about $5 \%$ dissociation of ${ }^{18} \mathrm{~F}$ from UCNPs was

15 observed, with a potentially negative effect on the toxicity and on the imaging quality. ${ }^{149}$ Theoretically, this last problem could be avoided - with one-step synthesis of the UCNPs - using ${ }^{18} \mathrm{~F}^{-}$ as a reagent for the preparation of the NP core; unfortunately not a possible option since the synthesis would require more half-life. To address this issue ${ }^{124} \mathrm{I}$, a radionuclide with a halflife of 4.18 days, has been used to derivatize the RGD peptide conjugated with $30 \mathrm{~nm} \mathrm{NaGdF}$ : $\mathrm{Yb}^{3+} / \mathrm{Er}^{3+} \mathrm{UCNPs}$, preparing in this way a trimodal PET/MR/optical probe. These NPs showed thanks to the targeting properties of the RGD peptide - a high specificity for $\alpha_{\mathrm{v}} \beta_{3}$ integrin-expressing U87MG tumor cells and xenografted tumor models. ${ }^{150}$ Within the lanthanide-based NPs, a more general possibility for inserting a radionuclide with sufficiently long half-life is represented by ${ }^{153} \mathrm{Sm}$, which has a half-time of $46.3 \mathrm{~h}$, thus offering the possibility to overcome the main limitations encountered by the use of ${ }^{18} \mathrm{~F}$.

${ }^{153} \mathrm{Sm}$, emitting beta particles and gamma rays $\left(E_{\gamma}=103 \mathrm{keV}\right)$, allows SPECT imaging.

Furthermore, ${ }^{153} \mathrm{Sm}^{3+}$ shows chemical properties, including the ionic radius, similar to the other lanthanide $\mathrm{Ln}^{3+}$ ions, and it can thus be easily used for doping Ln-based nanoparticles. In addition, because of the high sensitivity typical of the SPECT imaging, the low amount of ${ }^{153} \mathrm{Sm}^{3+}$ that is required does not alter substantially the properties of the host structure includ0 ing, if any, its UC ability. An interesting example is represented by $\mathrm{NaLuF}_{4}:{ }^{153} \mathrm{Sm}, \mathrm{Yb}, \mathrm{Tm}$ nanoparticles, obtained by hydrothermal synthesis by Y. Yang and coworkers. ${ }^{149}$ These NPs, with a 25-30 nm diameter and capped with OA and 6-aminohexanoic acid, showed a UC luminescence higher than that observed the first time in the imaging of normal Kunming mice with rich fur in vivo. Using the same NPs, SPECT images were taken at 1 and $24 \mathrm{~h}$ after injection. Also in this case, as it can be seen from Fig. 20, an intense signal was observed in the liver and in the spleen, in agreement with UCL images. At $24 \mathrm{~h}$, the authors observed a much larger uptake by the spleen compared to the liver, a result that can be partially explained by the fact that spleen is the largest organ of the immune system.

A different, and easier approach for the synthesis of SPECT active NPs has been successively proposed by the same group, based on a post-labelling procedure through a cation exchange
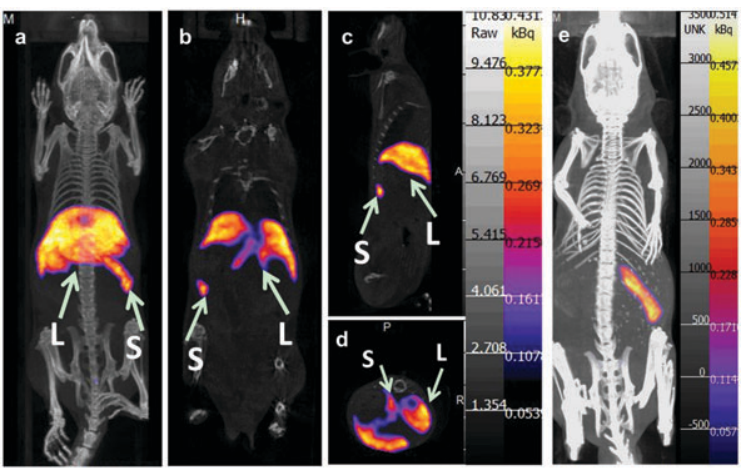

1

Fig. 20 In vivo SPECT images after intravenous injection of Sm-UCNPS. (a) Whole-body three-dimensional projection, (b) coronal, (c) sagittal and (d) transversal images acquired at $1 \mathrm{~h}$ and (e) whole-body threedimensional projection images acquired at $24 \mathrm{~h}$ are shown respectively. The arrows inset point to the liver (L) and spleen (S). Adapted from ref. 149 Copyright 2011 Elsevier Ltd.

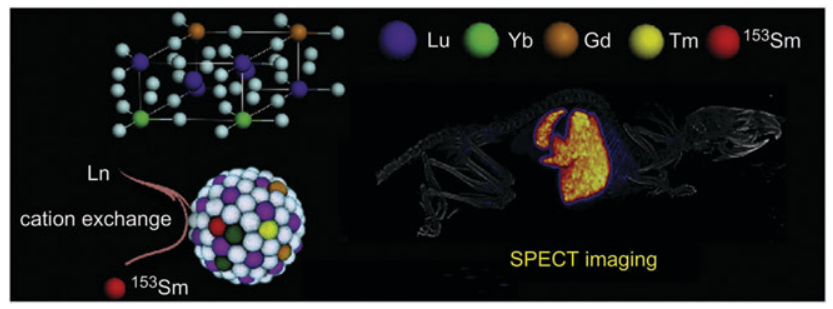

Fig. 21 Schematic representation of ${ }^{153} \mathrm{Sm}$-postlabeled citrate-capped $\mathrm{NaLuF}_{4}: \mathrm{Yb}, \mathrm{Gd}$,Tm by cation exchange for in vivo quantitative tracking. Adapted from ref. 188 Copyright 2013 Elsevier Ltd.

method, as schematized in Fig. $21 .^{188}$ In this case, citratecapped $\mathrm{NaLuF}_{4}: \mathrm{Yb}, \mathrm{Gd}$,Tm were mixed with ${ }^{153} \mathrm{SmCl}_{3}$ aqueous solutions; the precipitate obtained after a very short shaking (less than $1 \mathrm{~min}$ ) at room temperature was collected by centrifugation.

Almost no dissociation of ${ }^{153} \mathrm{Sm}^{3+}$ from the nanoparticles was observed, even after storage in fetal bovine serum for $72 \mathrm{~h}$. Moreover, when injected into mice, only a negligible ${ }^{153} \mathrm{Sm}^{3+}$ signal was observed in urine (by large, the main excretion route for the free ion) even at $300 \mathrm{~min}$ after injection, indicating a negligible dissociation also in vivo. The results obtained showed that this synthetic strategy could be used to prepare efficient dual-mode (UCL and SPECT) nanoparticles with a very short labelling time (less than $1 \mathrm{~min}$ ). Interestingly this strategy could be extended to other series of rare-earth nanoparticles, including phosphates, fluorides, and oxides of rare-earth ions, offering in all cases high labelling yield and stability.

Taking advantage of the two described synthetic methods, slightly different materials were realized for a very interesting pre-clinical application: blood pool imaging in vivo. In a first case RE-based nanoprobes constituted by very small (sub-10 $\mathrm{nm}$ ) PEG-coated UCNPs doped with ${ }^{153} \mathrm{Sm}^{3+}$ were prepared using a hydrothermal synthetic procedure. ${ }^{149}$ The SPECT imaging clearly indicated that these NPs have a long blood retention time in vivo and, importantly, that the renal excretion was 
1 in this case more rapid than the hepatobiliary excretion in the faeces. In a second example, larger $(20 \mathrm{~nm})$ radioactive/upconverting $\mathrm{NaLuF}_{4}: \mathrm{Yb}, \mathrm{Tm},{ }^{153} \mathrm{Sm}$ NPs, coated with ethylenediamine tetramethylenephosphonic acid (EDTMP), were synthesized via a solvothermal method, with the advantage of a reduced time consumption with respect to the previous example. However, as expected because of their larger size, a remarkable accumulation of these NPs in liver and in spleen could be observed at $1 \mathrm{~h}$ after injection.

\subsection{X-ray CT}

As already mentioned in the Introduction, also X-ray CT is a widely used imaging technique in medicine. In this case, together with barium salts, non-ionic, low-osmolar iodinated small molecules as iobitridol are used as contrast agents in 15 clinical applications. These molecules, however, suffer from short circulation lifetime and renal toxicity. ${ }^{189,190}$ Y. Liu et al. ${ }^{189}$ demonstrated that $\mathrm{NaYbF}_{4}$ :Er NPs, when doped with $\mathrm{Gd}^{3+}$ concentration of $20 \mathrm{~mol} \%$ or higher (to obtain a better control over size and shape) and capped with a PEG derivative, could be very promising for this technique. As a matter of fact, these NPs showed a higher CT contrast signal under the normal operating conditions of clinical imagers $(120 \mathrm{kVp})$ not only in comparison with a solution having the same concentration $\left(\mathrm{g} \mathrm{mL}^{-1}\right)$ of iobitridol, but also with respect to salts of heavy metals (Au, Pt, $\mathrm{Bi}$, and $\mathrm{Ta})$. The K-edge of $\mathrm{Yb}(61 \mathrm{keV})$ is in fact located just within the higher energy region of the X-ray spectrum obtained with the usual clinical setting. It has to be very briefly recalled here that the binding energy of the $\mathrm{K}$ shell electrons in an atom (K-edge) has to be at least paired, or better a bit exceeded, by the energy of the photon to be absorbed. In contrast, the K-edge values of $\mathrm{Au}, \mathrm{Pt}, \mathrm{Bi}$, and Ta were found to be far from the region of clinical imagers, thus revealing that Ln-based probes could be among the most effective in vivo CT contrast agents at $120 \mathrm{kVp}$.

A rare example of a dual probe for SPECT and X-ray CT 35 imaging was based on hydrophilic PEG coated EuOF: ${ }^{153} \mathrm{Sm}$ NPs. ${ }^{191}$ In this case, together with the imaging modality given by the presence of ${ }^{153} \mathrm{Sm}^{3+}$ as a dopant, the authors took profit of the high degree of X-ray absorption of europium. These NPs, having a diameter core of about $5 \mathrm{~nm}$, showed indeed a the same concentration) CT contrast signal, as shown in Fig. 22 in Hounsfield units (HU).

This figure shows CT images acquired $20 \mathrm{~min}$ after the injection into the left paw of a mouse of $150 \mu \mathrm{L}$ of the NPs at

45 a concentration of $4.0 \mathrm{mg} \mathrm{mL}^{-1}$. As can be seen, a significant contrast enhancement of the lymph node could be observed, extending in this way the applications of these NPs. SPECT images were conveniently taken with the same nanostructures for determining their dynamic distribution in vivo, indicating a blood lifetime of $4.65 \mathrm{~h}$, and an efficient elimination through biliary/gastrointestinal pathway. As already mentioned, the ability to efficiently absorb X-rays is common to all the RE elements, and for this reason it has been used in other cases, for example with $\mathrm{Gd}_{2} \mathrm{O}_{3}: \mathrm{Yb}^{3+}, \mathrm{Er}^{3+} \mathrm{UC}$ nanorods. ${ }^{192}$ In some cases, the presence of additional heavy atoms, such as $\mathrm{Ba}^{2+}$ in the $\mathrm{BaGdF}_{5}: \mathrm{Yb} / \mathrm{Er}$ NPs, could even increase the CT contrast. ${ }^{193}$

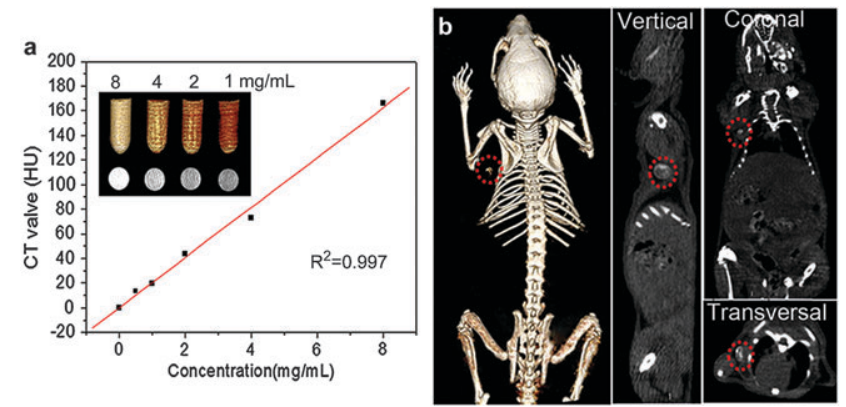

1

Fig. 22 (a) Hounsfield unit (HU) measurements of PEG-EuOF with different mass concentration of 1.0, 2.0, 4.0, and $8.0 \mathrm{mg} \mathrm{mL}^{-1}$. Inset: phantom and color-mapped CT images of PEG-EUOF with different mass concentration. (b) In vivo CT volume-rendered and intensity of vertical, coronal, transversal images of $20 \mathrm{~min}$ after injection of $4.0 \mathrm{mg} \mathrm{mL}^{-1}$ PEG-EuOF. The position of lymph node was marked by circles. Adapted from ref. 191 Copyright 2014 Elsevier Ltd.

\subsection{Photoacoustic imaging}

We would like to conclude this section with the example of a nanoprobe for photoacoustic imaging (PAI), a very interesting and promising imaging modality, with very significant advantages such as high resolution $(10-100 \times v$ s. PET), deeper tissue penetration compared to OI, and cost-effectiveness compared to SPECT, PET, and MRI. ${ }^{194,195}$ In particular PAI requires a very efficient absorption in the NIR region; the instrumentation detects the ultrasound waves generated by the thermoelastic expansion of the medium surrounding the probe caused by the heat dissipation associated with the non-radiative deactivation processes of the excited state.

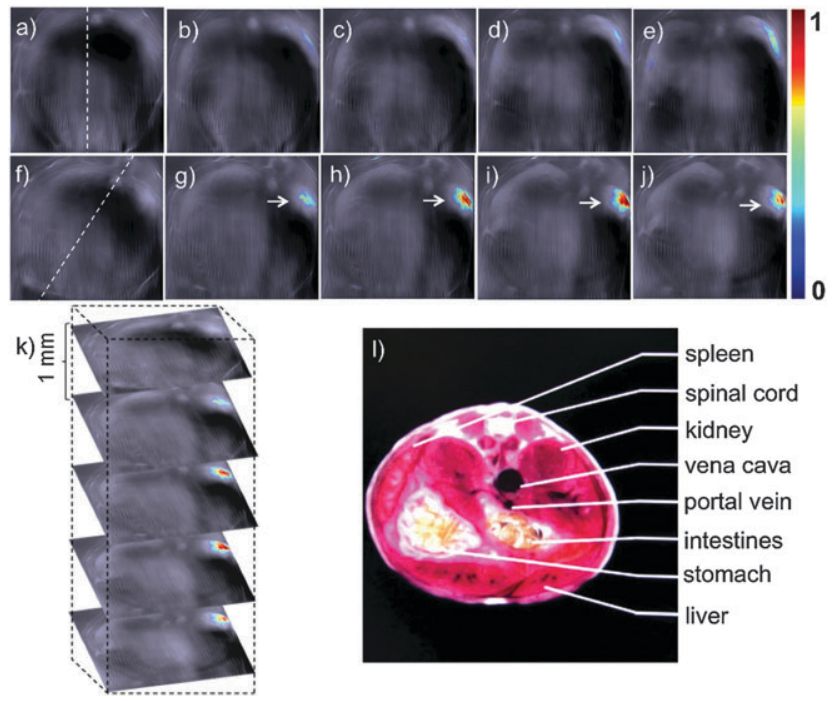

Fig. 23 Single-wavelength PAl of a live mouse anatomy at $980 \mathrm{~nm}$. (a-e) Individual anatomy sections of the live mouse before intravenous injection of $U C-\alpha-C D$. (f-j) Individual anatomy sections recorded after 35 min postintravenous injection of $U C-\alpha-C D$. Dashed lines in figure (a) and (f) indicate the positions of the mouse with respect to the viewer. Pointed areas in figure $(\mathrm{g}-\mathrm{j})$ indicate the localization of $\mathrm{UC}-\alpha-\mathrm{CD}$. (k) Three-dimensional rendering of scanned area. (I) Schematic section corresponding to analyzed area. Adapted from ref. 196 Copyright 2014 Wiley-VCH Verlag GmbH \& Co. KGaA, Weinheim. 
To our knowledge, the only example reported so far is based on OA-stabilized hexagonal-phased $\mathrm{NaYF}_{4}: \mathrm{Yb}^{3+}, \mathrm{Er}^{3+}(80: 18: 2)$ UCNPs dispersed in water. ${ }^{196} \mathrm{Yu}$ and co-workers solubilised these nanocrystals by $\alpha$-CD addition exploiting supramolecular alkyl chain/ $\alpha$-cyclodextrin $(\alpha-\mathrm{CD})$ host-guest association. The considerable luminescence quenching observed when dispersing the NPs in water (circa 62\%) had the effect of enhancing the PA signal, although the possibility of performing UCL and PAI at the same time can be in general considered a precious advantage. PAI imaging in vivo of an anesthetized mouse showed a clear contrast enhancement localized on the kidney (Fig. 23), proving that these NPs could be used as efficient contrast agents for diagnostic purposes under $980 \mathrm{~nm}$ excitation.

\section{Conclusions}

In this review we have tried to guide the reader through the recent advancements in the research on lanthanide-doped NPs for bioimaging applications, a field where they display tremendous potential. The unique characteristics of these species have been discussed via many examples, reporting the different approaches used in the various imaging techniques for both in vitro and in vivo (small animals) experiments. Summarising the information reported in Tables 1 and 2 and discussed for the different systems, we here suggest a schematic outlook of the strengths and weaknesses of these nanoparticles as imaging agents, comparing them with their molecular counterparts.

The clinical translation of optical imaging is in its early infancy; in this scenario lanthanide based nanomaterials present a quite unique property: the possibility of obtaining upconversion luminescence. This is a precious way to shift the absorption toward the NIR region, the most suitable one for biomedical applications. On the other side, exploiting the more common down shifting luminescence processes takes to systems presenting both the absorption and emission in the NIR region, and this approach is more and more attracting the scientific efforts of the field. Nevertheless for a clinical translation, lanthanide-doped NPs, even presenting a high photostability, need further investigation to improve some still critical features. In particular the brightness of UCNPs that is generally quite low (due to parasite cross-relaxations and narrow and low extinction coefficients), possible batch-to-batch variability in emission efficiency, the ace functionalization or a multi-material core-shell design to obtain suitable retention times and efficient clearance when in vivo. A direct comparison with the performance of lanthanide complexes is difficult since their application is still quite far and rare also in living cells and their clinical translation is a very distant goal. This is due to many factors, among them the fact that many properties, especially in UCNPs, take profit of collective processes that are not possible in molecular structures. In addition, we would like to mention their blinking problems and the complexity of lanthanide photophysics that, being dramatically affected by the environment, often rises unpredicted problems in complex matrixes.
MRI contrast agents in the form of Gd-chelates represent an established clinical application of lanthanide based molecules. This topic has been given due prominence in the review, highlighting how it is possible to obtain a great boost in relaxivity through scaffolds that accommodate a large number of paramagnetic complexes on a single macromolecule of nanoscopic size. In this context dendrimers, monolayer-protected nanoparticles and virus capsids have provided perhaps the best results in terms of relaxivity gains. On the other side, when the gadolinium ions become part of a nanoparticle structure, the relaxivity gains are generally lower than the typical values of Gd-chelates conjugated to macromolecules. This evidence is mostly due to the physicochemical properties of lanthanide-doped nanoparticles and, together with other physiological factors such as the excretion times and the viscosity/osmolarity of injectable preparations, it may represent a limitation to the practical use of these systems.

Another valuable characteristic of lanthanide-doped nanoplatforms is the possibility to obtain, with relatively versatile approaches, multimodal imaging. The examples discussed in this review clearly show the advantages of a multimodal approach that allows co-localization and multiple targeting at the same time. These valuable features are not possible at the molecular level and very difficult to obtain (usually with very tedious synthetic procedures) also following a supramolecular approach.

It has to be mentioned that lanthanide based NPs also allow a further degree of complexity and with a relatively facile design it is also possible to obtain systems for drug delivery and light activated therapies, in particular with high penetrating NIR radiation. This is opening up the way to the preparation of theranostic agents with remarkable qualities in terms of biocompatibility, low toxicity, targeting and stimuli-regulated drug release.

All together these NPs are expected to play a fundamental role in medical diagnosis and therapy in the future since they merge capabilities and advantages coming from different materials, strategies and from the knowhow of fields such as chemistry, physics, biology and medicine.

\section{Acknowledgements}

This work was supported by: MIUR (PON 01_01078 granted to L.P.); University of Bologna (FARB project A.10.N2.RICER.FARB2RODAA granted to LP, FARB project linea1. NANOX granted to NZ); Fondazione Cariverona (Verona, Italy, "Verona Nanomedicine" Initiative granted to A.S.); and Università degli Studi di Padova (PRAT CPDA132473/13, granted to F.R.).

\section{Notes and references}

1 F. Pene, E. Courtine, A. Cariou and J. P. Mira, Crit. Care Med., 2009, 37, S50.

2 Q. F. Xiao, X. P. Zheng, W. B. Bu, W. Q. Ge, S. J. Zhang, F. Chen, H. Y. Xing, Q. G. Ren, W. P. Fan, K. L. Zhao, Y. Q. Hua and J. L. Shi, J. Am. Chem. Soc., 2013, 135, 13041.

3 C. Y. Chen, Y. F. Li, Y. Qu, Z. F. Chai and Y. L. Zhao, Chem. Soc. Rev., 2013, 42, 8266.
1

5 
4 R. Weissleder, M. Nahrendorf and M. J. Pittet, Nat. Mater., 2014, 13, 125.

5 T. Hussain and Q. T. Nguyen, Adv. Drug Delivery Rev., 2014, 66, 90.

6 S. Kaur, G. Venktaraman, M. Jain, S. Senapati, P. K. Garg and S. K. Batra, Cancer Lett., 2012, 315, 97.

7 V. Biju, Chem. Soc. Rev., 2014, 43, 744.

8 H. Gong, R. Peng and Z. Liu, Adv. Drug Delivery Rev., 2013, 65, 1951.

9 F. Novio, J. Simmchen, N. Vazquez-Mera, L. Amorin-Ferre and D. Ruiz-Molina, Coord. Chem. Rev., 2013, 257, 2839.

10 W. Arap, R. Pasqualini, M. Montalti, L. Petrizza, L. Prodi, E. Rampazzo, N. Zaccheroni and S. Marchio, Curr. Med. Chem., 2013, 20, 2195.

11 J. M. Warram, E. de Boer, A. G. Sorace, T. K. Chung, H. Kim, R. G. Pleijhuis, G. M. van Dam and E. L. Rosenthal, Cancer Metastasis Rev., 2014, 33, 809.

12 Y. Xing, J. Zhao, P. S. Conti and K. Chen, Theranostics, 2014, 4, 290.

13 J. Gallo, N. J. Long and E. O. Aboagye, Chem. Soc. Rev., 2013, 42, 7816.

14 N. Lee, S. H. Choi and T. Hyeon, Adv. Mater., 2013, 25, 2641.

15 N. Mitchell, T. L. Kalber, M. S. Cooper, K. Sunassee, S. L. Chalker, K. P. Shaw, K. L. Ordidge, A. Badar, S. M. Janes, P. J. Blower, M. F. Lythgoe, H. C. Hailes and A. B. Tabor, Biomaterials, 2013, 34, 1179.

16 F. Kiessling, S. Fokong, J. Bzyl, W. Lederle, M. Palmowski and T. Lammers, Adv. Drug Delivery Rev., 2014, 72, 15.

17 J. Unga and M. Hashida, Adv. Drug Delivery Rev., 2014, 72, 144.

18 S. Zackrisson, S. M. van de Ven and S. S. Gambhir, Cancer Res., 2014, 74, 979.

19 Q. Liu, W. Feng and F. Y. Li, Coord. Chem. Rev., 2014, 273, 100 .

20 E. Hemmer, N. Venkatachalam, H. Hyodo, A. Hattori, Y. Ebina, H. Kishimoto and K. Soga, Nanoscale, 2013, 5, 11339.

21 Z. Hou, Y. Zhang, K. Deng, Y. Chen, X. Li, X. Deng, Z. Cheng, H. Lian, C. Li and J. Lin, ACS Nano, 2015, 9, 2584.

22 Y. Li, T.-y. Lin, Y. Luo, Q. Liu, W. Xiao, W. Guo, D. Lac, H. Zhang, C. Feng, S. Wachsmann-Hogiu, J. H. Walton, S. R. Cherry, D. J. Rowland, D. Kukis, C. Pan and K. S. Lam, Nat. Commun., 2014, 5.

23 R. Vankayala, Y. K. Huang, P. Kalluru, C. S. Chiang and K. C. Hwang, Small, 2014, 10, 1612.

24 M. Guo, H. J. Mao, Y. L. Li, A. J. Zhu, H. He, H. Yang, Y. Y. Wang, X. Tian, C. C. Ge, Q. L. Peng, X. Y. Wang, X. L. Yang, X. Y. Chen, G. Liu and H. B. Chen, Biomaterials, 2014, 35, 4656.

25 B. K. Wang, J. H. Wang, Q. Liu, H. Huang, M. Chen, K. Y. Li, C. Z. Li, X. F. Yu and P. K. Chu, Biomaterials, 2014, 35, 1954.

26 G. Terentyuk, E. Panfilova, V. Khanadeev, D. Chumakov, E. Genina, A. Bashkatov, V. Tuchin, A. Bucharskaya, G. Maslyakova, N. Khlebtsov and B. Khlebtsov, Nano Res., 2014, 7, 325.
28 E. Rampazzo, S. Bonacchi, D. Genovese, R. Juris, M. Montalti, V. Paterlini, N. Zaccheroni, C. Dumas-Verdes, G. Clavier, R. Meallet-Renault and L. Prodi, J. Phys. Chem. C, 2014, 118, 9261.

29 N. Khemthongcharoen, R. Jolivot, S. Rattanavarin and W. Piyawattanametha, Adv. Drug Delivery Rev., 2014, 74, 53.

30 R. R. Anderson and J. A. Parrish, J. Invest. Dermatol., 1981, 77, 13.

31 U. Rocha, K. U. Kumar, C. Jacinto, I. Villa, F. Sanz-Rodríguez, M. del Carmen Iglesias de la Cruz, A. Juarranz, E. Carrasco, F. C. J. M. van Veggel, E. Bovero, J. G. Solé and D. Jaque, Small, 2014, 10, 1141.

32 R. Wang and F. Zhang, J. Mater. Chem. B, 2014, 2, 2422.

33 D. Yang, P. a. Ma, Z. Hou, Z. Cheng, C. Li and J. Lin, Chem. Soc. Rev., 2014, 44, 1416.

34 L. Z. Zhao, J. J. Peng, Q. Huang, C. Y. Li, M. Chen, Y. Sun, Q. N. Lin, L. Y. Zhu and F. Y. Li, Adv. Funct. Mater., 2014, 24, 363.

35 M. V. DaCosta, S. Doughan, Y. Han and U. J. Krull, Anal. Chim. Acta, 2014, 832, 1.

36 Y. Liu, D. Tu, H. Zhu and X. Chen, Chem. Soc. Rev., 2013, 42, 6924.

37 J. Zhou, Z. Liu and F. Li, Chem. Soc. Rev., 2012, 41, 1323.

38 C. F. Gainer and M. Romanowski, J. Innovative Opt. Health Sci., 2014, 07, 1330007.

39 A. Sedlmeier and H. H. Gorris, Chem. Soc. Rev., 2014, 44, 1526.

40 M.-K. Tsang, G. Bai and J. Hao, Chem. Soc. Rev., 2015, 44, 1585.

41 W. Zheng, P. Huang, D. Tu, E. Ma, H. Zhu and X. Chen, Chem. Soc. Rev., 2015, 44, 1379.

42 S. L. Gai, C. X. Li, P. P. Yang and J. Lin, Chem. Rev., 2014, 114, 2343.

43 F. C. J. M. van Veggel, C. Dong, N. J. J. Johnson and J. Pichaandi, Nanoscale, 2012, 4, 7309.

44 L. D. Sun, Y. F. Wang and C. H. Yan, Acc. Chem. Res., 2014, 47, 1001.

45 N. M. Idris, M. K. G. Jayakumar, A. Bansal and Y. Zhang, Chem. Soc. Rev., 2014, 44, 1449.

46 E. Pershagen and K. E. Borbas, Coord. Chem. Rev., 2014, 273,30 .

47 L. J. Xu, G. T. Xu and Z. N. Chen, Coord. Chem. Rev., 2014, 273, 47.

48 G. Liu, Chem. Soc. Rev., 2015, 44, 1635.

49 Y. Sun, W. Feng, P. Yang, C. Huang and F. Li, Chem. Soc. Rev., 2015.

50 A. Gnach, T. Lipinski, A. Bednarkiewicz, J. Rybka and J. A. Capobianco, Chem. Soc. Rev., 2015, 44, 1561.

51 N. Bloembergen, Phys. Rev. Lett., 1959, $2,84$.

52 F. Auzel, Chem. Rev., 2004, 104, 139.

53 J. S. Chivian, W. E. Case and D. D. Eden, Appl. Phys. Lett., 1979, 35, 124.

54 M. F. Joubert, Opt. Mater., 1999, 11, 181.

55 Y. Zhang, W. Wei, G. K. Das and T. T. Y. Tan, J. Photochem. Photobiol., C, 2014, 20, 71.

56 H. J. M. A. A. Zijlmans, J. Bonnet, J. Burton, K. Kardos, T. Vail, R. S. Niedbala and H. J. Tanke, Anal. Biochem., 1999, 267, 30. 2013, 52, 3584 . 
157 X. Li, F. Zhang and D. Zhao, Chem. Soc. Rev., 2014, 44, 1346.

58 X. Chen, D. Peng, Q. Ju and F. Wang, Chem. Soc. Rev., 2014, 44, 1318.

59 X. Yan, G. R. Fern, R. Withnall and J. Silver, Nanoscale, 2013, 5, 1091.

60 S. Polizzi, S. Bucella, A. Speghini, F. Vetrone, R. Naccache, J. C. Boyer and J. A. Capobianco, Chem. Mater., 2004, 16, 1330.

61 F. Vetrone, J. C. Boyer, J. A. Capobianco, A. Speghini and M. Bettinelli, Nanotechnology, 2004, 15, 75.

62 E. Nakazawa and S. Shionoya, Phys. Rev. Lett., 1970, 25, 1710 .

63 F. Wang, R. R. Deng, J. Wang, Q. X. Wang, Y. Han, H. M. Zhu, X. Y. Chen and X. G. Liu, Nat. Mater., 2011, 10, 968.

64 L. Tu, X. Liu, F. Wu and H. Zhang, Chem. Soc. Rev., 2015, 44, 1331.

65 H. Dong, L.-D. Sun and C.-H. Yan, Chem. Soc. Rev., 2015, 44, 1608.

66 G. Y. Chen, C. H. Yang and P. N. Prasad, Acc. Chem. Res., 2013, 46, 1474.

67 Q. Q. Su, S. Y. Han, X. J. Xie, H. M. Zhu, H. Y. Chen, C. K. Chen, R. S. Liu, X. Y. Chen, F. Wang and X. G. Liu, J. Am. Chem. Soc., 2012, 134, 20849.

68 C. T. Xu, Q. Q. Zhan, H. C. Liu, G. Somesfalean, J. Qian, S. L. He and S. Andersson-Engels, Laser Photonics Rev., 2013, 7, 663.

69 W. Yin, L. Zhao, L. Zhou, Z. Gu, X. Liu, G. Tian, S. Jin, L. Yan, W. Ren, G. Xing and Y. Zhao, Chem. - Eur. J., 2012, 18, 9239.

70 T. Yang, Y. Sun, Q. Liu, W. Feng, P. Yang and F. Li, Biomaterials, 2012, 33, 3733.

71 A. Gnach, K. Prorok, M. Misiak, B. Cichy and A. Bednarkiewicz, J. Rare Earths, 2014, 32, 207.

72 G. Y. Chen, T. Y. Ohulchanskyy, R. Kumar, H. Agren and P. N. Prasad, ACS Nano, 2010, 4, 3163.

73 J. Ryu, H. Y. Park, K. Kim, H. Kim, J. H. Yoo, M. Kang, K. Im, R. Grailhe and R. Song, J. Phys. Chem. C, 2010, 114, 21077.

74 G. Gao, C. L. Zhang, Z. J. Zhou, X. Zhang, J. B. Ma, C. Li, W. L. Jin and D. X. Cui, Nanoscale, 2013, 5, 351.

75 M. Pedroni, F. Piccinelli, T. Passuello, S. Polizzi, J. Ueda, P. Haro-Gonzalez, L. M. Maestro, D. Jaque, J. Garcia-Sole, M. Bettinelli and A. Speghini, Cryst. Growth Des., 2013, 13, 4906.

76 I. X. Cantarelli, M. Pedroni, F. Piccinelli, P. Marzola, F. Boschi, G. Conti, A. Sbarbati, P. Bernardi, E. Mosconi, L. Perbellini, L. Marongiu, M. Donini, S. Dusi, L. Sorace, C. Innocenti, E. Fantechi, C. Sangregorio and A. Speghini, Biomater. Sci., 2014, 2, 1158.

77 J. Liu, Y. Liu, W. Bu, J. Bu, Y. Sun, J. Du and J. Shi, J. Am. Chem. Soc., 2014, 136, 9701.

78 L. Liu, F. Qin, H. Zhao, T. Lv, Z. Zhang and W. Cao, Opt. Lett., 2013, 38, 2101.

79 J. A. Damasco, G. Chen, W. Shao, H. Agren, H. Huang, W. Song, J. F. Lovell and P. N. Prasad, ACS Appl. Mater. Interfaces, 2014, 6, 13884.
80 A. Priyam, N. M. Idris and Y. Zhang, J. Mater. Chem., 2012, 22, 960.

81 Z. Li, S. Lv, Y. Wang, S. Chen and Z. Liu, J. Am. Chem. Soc., 2015, 137, 3421.

82 J. Peng, W. Xu, C. L. Teoh, S. Han, B. Kim, A. Samanta, J. C. Er, L. Wang, L. Yuan, X. Liu and Y.-T. Chang, J. Am. Chem. Soc., 2015, 137, 2336.

83 T. Y. Cao, Y. Yang, Y. A. Gao, J. Zhou, Z. Q. Li and F. Y. Li, Biomaterials, 2011, 32, 2959.

84 L. C. Ong, L. Y. Ang, S. Alonso and Y. Zhang, Biomaterials, 2014, 35, 2987.

85 D. Ni, J. Zhang, W. Bu, H. Xing, F. Han, Q. Xiao, Z. Yao, F. Chen, Q. He, J. Liu, S. Zhang, W. Fan, L. Zhou, W. Peng and J. Shi, ACS Nano, 2014, 8, 1231.

86 X. Zhu, B. Da Silva, X. Zou, B. Shen, Y. Sun, W. Feng and F. Li, RSC Adv., 2014, 4, 23580.

87 Y. H. Chien, Y. L. Chou, S. W. Wang, S. T. Hung, M. C. Liau, Y. J. Chao, C. H. Su and C. S. Yeh, ACS Nano, 2013, 7, 8516.

88 C. Wang, L. Cheng, Y. Liu, X. Wang, X. Ma, Z. Deng, Y. Li and Z. Liu, Adv. Funct. Mater., 2013, 23, 3077.

89 R. J. McNichols, A. Gowda, M. Kangasniemi, J. A. Bankson, R. E. Price and J. D. Hazle, Lasers Surg. Med., 2004, 34, 48.

$90 \mathrm{~J}$. Torres-Reveron, H. Tomasiewicz, A. Shetty, N. Amankulor and V. Chiang, J. Neurooncol., 2013, 113, 495.

91 Q. Zhan, J. Qian, H. Liang, G. Somesfalean, D. Wang, S. He, Z. Zhang and S. Andersson-Engels, ACS Nano, 2011, 5,3744 .

92 G. Y. Chen, T. Y. Ohulchanskyy, A. Kachynski, H. Agren and P. N. Prasad, ACS Nano, 2011, 5, 4981.

93 J. Shen, G. Y. Chen, A. M. Vu, W. Fan, O. S. Bilsel, C. C. Chang and G. Han, Adv. Opt. Mater., 2013, 1, 644.

94 Y. F. Wang, G. Y. Liu, L. D. Sun, J. W. Xiao, J. C. Zhou and C. H. Yan, ACS Nano, 2013, 7, 7200.

95 X. J. Xie, N. Y. Gao, R. R. Deng, Q. Sun, Q. H. Xu and X. G. Liu, J. Am. Chem. Soc., 2013, 135, 12608.

96 Y. Zhong, G. Tian, Z. Gu, Y. Yang, L. Gu, Y. Zhao, Y. Ma and J. Yao, Adv. Mater., 2014, 26, 2831.

97 A. Bednarkiewicz, D. Wawrzynczyk, M. Nyk and W. Strek, Opt. Mater., 2011, 33, 1481.

98 D. Wawrzynczyk, A. Bednarkiewicz, M. Nyk, W. Strek and M. Samoc, Nanoscale, 2013, 5, 12671.

99 M. C. Tan, G. A. Kumar, R. E. Riman, M. G. Brik, E. Brown and U. Hommerich, J. Appl. Phys., 2009, 106, 063118.

100 G. Y. Chen, T. Y. Ohulchanskyy, S. Liu, W. C. Law, F. Wu, M. T. Swihart, H. Agren and P. N. Prasad, ACS Nano, 2012, 6, 2969.

101 M. Y. Xie, L. Yu, H. He and X. F. Yu, J. Solid State Chem., 2009, 182, 597.

102 H.-S. Qian and Y. Zhang, Langmuir, 2008, 24, 12123.

103 I. Villa, A. Vedda, I. Cantarelli, M. Pedroni, F. Piccinelli, M. Bettinelli, A. Speghini, M. Quintanilla, F. Vetrone, U. Rocha, C. Jacinto, E. Carrasco, F. Rodríguez, Á. Juarranz, B. del Rosal, D. Ortgies, P. Gonzalez, J. Solé and D. García, Nano Res., 2014, 1.
1 
1104 U. Rocha, C. Jacinto, W. F. Silva, I. Guedes, A. Benayas, L. M. Maestro, M. A. Elias, E. Bovero, F. C. J. M. van Veggel, J. A. G. Sole and D. Jaque, ACS Nano, 2013, 7, 1188.

105 D. Cauzzi, R. Pattacini, M. Delferro, F. Dini, C. Di Natale, R. Paolesse, S. Bonacchi, M. Montalti, N. Zaccheroni, M. Calvaresi, F. Zerbetto and L. Prodi, Angew. Chem., Int. Ed., 2012, 51, 9662.

106 M. Pokhrel, L. C. Mimun, B. Yust, G. A. Kumar, A. Dhanale, L. Tang and D. K. Sardar, Nanoscale, 2014, 6, 1667.

107 L. C. Mimun, G. Ajithkumar, M. Pokhrel, B. G. Yust, Z. G. Elliott, F. Pedraza, A. Dhanale, L. Tang, A. L. Lin, V. P. Dravid and D. K. Sardar, J. Mater. Chem. B, 2013, 1, 5702 .

108 F. Liu, Q. Zhao, H. You and Z. Wang, Nanoscale, 2013, $5,1047$.

109 X. Li, R. Wang, F. Zhang, L. Zhou, D. Shen, C. Yao and D. Zhao, Sci. Rep., 2013, 3, 3536.

110 P. C. Lauterbur, Nature, 1973, 242, 190.

111 M. Botta and L. Tei, Eur. J. Inorg. Chem., 2012, 1945.

112 I. Bertini, C. Luchinat and G. Parigi, Solution NMR of Paramagnetic Molecules: Applications to metallobiomolecules and models, 2001.

113 J. Kowalewski and L. Mäler, Nuclear Spin Relaxation in Liquids: Theory, Experiments, and Applications, CRC Press, 2006.

114 M. H. Levitt, Spin Dynamics: Basics of Nuclear Magnetic Resonance, John Wiley \& Sons Ltd, Chichester, 2nd edn, 2007.

115 F. Carniato, K. Thangavel, L. Tei and M. Botta, J. Mater. Chem. B, 2013, 1, 2442.

116 D. Kruk and J. Kowalewski, J. Magn. Reson., 2003, 162, 229.

117 P. Caravan, J. J. Ellison, T. J. McMurry and R. B. Lauffer, Chem. Rev., 1999, 99, 2293.

118 M. Botta, Eur. J. Inorg. Chem., 2000, 399.

119 Y. Ayant, E. Belorizky, J. Aluzon and J. Gallice, J. Phys., 1975, 36, 991.

120 B. Halle, J. Chem. Phys., 2003, 119, 12372.

121 D. Frezzato, F. Rastrelli and A. Bagno, J. Phys. Chem. B, 2006, 110, 5676.

122 A. J. L. Villaraza, A. Bumb and M. W. Brechbiel, Chem. Rev., 2010, 110, 2921.

123 W. Xu, K. Kattel, J. Y. Park, Y. Chang, T. J. Kim and G. H. Lee, Phys. Chem. Chem. Phys., 2012, 14, 12687.

124 S. H. Lee, B. H. Kim, H. B. Na and T. Hyeon, Wiley Interdiscip. Rev.: Nanomed. Nanobiotechnol., 2014, 6, 196.

125 J. Y. Park, M. J. Baek, E. S. Choi, S. Woo, J. H. Kim, T. J. Kim, J. C. Jung, K. S. Chae, Y. Chang and G. H. Lee, ACS Nano, 2009, 3, 3663.

126 J. Y. Park, E. S. Choi, M. J. Baek, G. H. Lee, S. Woo and Y. Chang, Eur. J. Inorg. Chem., 2009, 2477.

127 E. S. Choi, J. Y. Park, M. J. Baek, W. Xu, K. Kattel, J. H. Kim, J. J. Lee, Y. Chang, T. J. Kim, J. E. Bae, K. S. Chae, K. J. Suh and G. H. Lee, Eur. J. Inorg. Chem., 2010, 4555.

128 M. Ahren, L. Selegard, A. Klasson, F. Soderlind, N. Abrikossova, C. Skoglund, T. Bengtsson, M. Engstrom, P.-O. Kall and K. Uvdal, Langmuir, 2010, 26, 5753.
129 L. Faucher, M. Tremblay, J. Lagueux, Y. Gossuin and M.A. Fortin, ACS Appl. Mater. Interfaces, 2012, 4, 4506.

130 H. Hifumi, S. Yamaoka, A. Tanimoto, T. Akatsu, Y. Shindo, A. Honda, D. Citterio, K. Oka, S. Kuribayashi and K. Suzuki, J. Mater. Chem., 2009, 19, 6393.

131 F. Evanics, P. R. Diamente, F. van Veggel, G. J. Stanisz and R. S. Prosser, Chem. Mater., 2006, 18, 2499.

132 N. J. J. Johnson, A. Korinek, C. Dong and F. C. J. M. van Veggel, J. Am. Chem. Soc., 2012, 134, 11068.

133 N. J. J. Johnson, W. Oakden, G. J. Stanisz, R. S. Prosser and F. C. J. M. van Veggel, Chem. Mater., 2011, 23, 3714.

134 F. Chen, W. B. Bu, S. J. Zhang, J. N. Liu, W. P. Fan, L. P. Zhou, W. J. Peng and J. L. Shi, Adv. Funct. Mater., 2013, 23, 298.

135 F. Chen, W. Bu, S. Zhang, X. Liu, J. Liu, H. Xing, Q. Xiao, L. Zhou, W. Peng, L. Wang and J. Shi, Adv. Funct. Mater., 2011, 21, 4285.

136 R. M. Petoral, Jr., F. Soderlind, A. Klasson, A. Suska, M. A. Fortin, N. Abrikossova, L. Selegard, P.-O. Kall, M. Engstrom and K. Uvdal, J. Phys. Chem. C, 2009, 113, 6913.

137 Y. Li, T. Chen, W. Tan and D. R. Talham, Langmuir, 2014, 30, 5873.

138 T. Passuello, M. Pedroni, F. Piccinelli, S. Polizzi, P. Marzola, S. Tambalo, G. Conti, D. Benati, F. Vetrone, M. Bettinelli and A. Speghini, Nanoscale, 2012, 4, 7682.

139 F. Chen, W. B. Bu, S. J. Zhang, X. H. Liu, J. N. Liu, H. Y. Xing, Q. F. Xiao, L. P. Zhou, W. J. Peng, L. Z. Wang and J. L. Shi, Adv. Funct. Mater., 2011, 21, 4285.

140 S. A. Osseni, S. Lechevallier, M. Verelst, P. Perriat, J. Dexpert-Ghys, D. Neumeyer, R. Garcia, F. Mayer, K. Djanashvili, J. A. Peters, E. Magdeleine, H. GrosDagnac, P. Celsis and R. Mauricot, Nanoscale, 2014, 6, 555.

141 A. Xia, M. Chen, Y. Gao, D. Wu, W. Feng and F. Li, Biomaterials, 2012, 33, 5394.

142 M. F. Dumont, H. A. Hoffman, P. R. S. Yoon, L. S. Conklin, S. R. Saha, J. Paglione, R. W. Sze and R. Fernandes, Bioconjugate Chem., 2014, 25, 129.

143 M. Mahmoudi, K. Azadmanesh, M. A. Shokrgozar, W. S. Journeay and S. Laurent, Chem. Rev., 2011, 111, 3407.

144 Q. Liu, M. Chen, Y. Sun, G. Y. Chen, T. S. Yang, Y. Gao, X. Z. Zhang and F. Y. Li, Biomaterials, 2011, 32, 8243.

145 J. Zhou, M. X. Yu, Y. Sun, X. Z. Zhang, X. J. Zhu, Z. H. Wu, D. M. Wu and F. Y. Li, Biomaterials, 2011, 32, 1148.

146 Y. Sun, M. X. Yu, S. Liang, Y. J. Zhang, C. G. Li, T. T. Mou, W. J. Yang, X. Z. Zhang, B. A. Li, C. H. Huang and F. Y. Li, Biomaterials, 2011, 32, 2999.

147 M. Helle, E. Rampazzo, M. Monchanin, F. Marchal, F. Guillemin, S. Bonacchi, F. Salis, L. Prodi and L. Bezdetnaya, ACS Nano, 2013, 7, 8645.

148 Q. Liu, Y. Sun, C. Li, J. Zhou, C. Li, T. Yang, X. Zhang, T. Yi, D. Wu and F. Li, ACS Nano, 2011, 5, 3146.

149 T. Cao, Y. Yang, Y. Sun, Y. Wu, Y. Gao, W. Feng and F. Li, Biomaterials, 2013, 34, 7127.

150 J. Lee, T. S. Lee, J. Ryu, S. Hong, M. Kang, K. Im, J. H. Kang, S. M. Lim, S. Park and R. Song, J. Nucl. Med., 2013, 54, 96.

151 J. G. Llorente, M. G. Gallego and A. M. Arnaiz, Acta Radiol., 1997, 38, 121. 
152 S. Aime, M. Botta, M. Panero, M. Grandi and F. Uggeri, Magn. Reson. Chem., 1991, 29, 923.

153 R. Shukla, M. Fernandez, R. K. Pilial, R. Ranganathan, P. C. Ratsep, X. Zhang and M. F. Tweedle, Magn. Reson. Med., 1996, 35, 928.

154 D. H. Powell, A. E. Merbach, G. González, E. Brücher, K. Micskei, M. F. Ottaviani, K. Köhler, A. Von Zelewsky, O. Y. Grinberg and Y. S. Lebedev, Helv. Chim. Acta, 1993, 76, 2129.

155 S. Aime, P. L. Anelli, M. Botta, F. Fedeli, M. Grandi, P. Paoli and F. Uggeri, Inorg. Chem., 1992, 31, 2422.

156 S. I. Kang, R. S. Ranganathan, J. E. Emswiler, K. Kumar, J. Z. Gougoutas, M. F. Malley and M. F. Tweedle, Inorg. Chem., 1993, 32, 2912.

157 X. Zhang, C. A. Chang, H. G. Brittain, J. M. Garrison, J. Telser and M. F. Tweedle, Inorg. Chem., 1992, 31, 5597.

158 J. Rudovský, M. Botta, P. Hermann, K. I. Hardcastle, I. Lukeš and S. Aime, Bioconjugate Chem., 2006, 17, 975.

159 T. Koullourou, L. S. Natrajan, H. Bhavsar, Pope, J. Feng, J. Narvainen, R. Shaw, E. Scales, R. Kauppinen, A. M. Kenwright and S. Faulkner, J. Am. Chem. Soc., 2008, 130, 2178.

160 G. Dehaen, S. V. Eliseeva, P. Verwilst, S. Laurent, L. Vander Elst, R. N. Muller, W. De Borggraeve, K. Binnemans and T. N. Parac-Vogt, Inorg. Chem., 2012, 51, 8775.

161 M. Querol, J. W. Chen, R. Weissleder and A. Bogdanov, Org. Lett., 2005, 7, 1719.

162 M. O. Breckwoldt, J. W. Chen, L. Stangenberg, E. Aikawa, E. Rodriguez, S. Qiu, M. A. Moskowitz and R. Weissleder, Proc. Natl. Acad. Sci. U. S. A., 2008, 105, 18584.

163 J. E. Jones, A. J. Amoroso, I. M. Dorin, G. Parigi, B. D. Ward, N. J. Buurma and S. J. A. Pope, Chem. Commun., 2011, 47, 3374 .

164 E. Debroye, M. Ceulemans, L. Vander Elst, S. Laurent, R. N. Muller and T. N. Parac-Vogt, Inorg. Chem., 2014, 53, 1257.

165 A. Keliris, T. Ziegler, R. Mishra, R. Pohmann, M. G. Sauer, K. Ugurbil and J. Engelmann, Bioorg. Med. Chem., 2011, 19, 2529.

166 G. Schuhmann-Giampieri, H. Schmitt-Willich, T. Frenzel, W.-R. Press and H.-J. Weinmann, Invest. Radiol., 1991, 26, 969.

167 C. T. Adkins, J. N. Dobish, C. S. Brown, B. Mayrsohn, S. K. Hamilton, F. Udoji, K. Radford, T. E. Yankeelov, J. C. Gore and E. Harth, Polym. Chem., 2012, 3, 390.

168 S. Laus, A. Sour, R. Ruloff, É. Tóth and A. E. Merbach, Chem. - Eur. J., 2005, 11, 3064.

169 P. D. Garimella, A. Datta, D. W. Romanini, K. N. Raymond and M. B. Francis, J. Am. Chem. Soc., 2011, 133, 14704.

170 L. Moriggi, C. Cannizzo, E. Dumas, C. R. Mayer, A. Ulianov and L. Helm, J. Am. Chem. Soc., 2009, 131, 10828.

171 D. Gerion, J. Herberg, R. Bok, E. Gjersing, E. Ramon, R. Maxwell, J. Kurhanewicz, T. F. Budinger, J. W. Gray, M. A. Shuman and F. F. Chen, J. Phys. Chem. C, 2007, 111, 12542.
172 L. Tei, G. Gugliotta, Z. Baranyai and M. Botta, Dalton Trans., 2009, 9712.

173 F. Kielar, L. Tei, E. Terreno and M. Botta, J. Am. Chem. Soc., 2010, 132, 7836.

174 L. M. Manus, D. J. Mastarone, E. A. Waters, X.-Q. Zhang, E. A. Schultz-Sikma, K. W. MacRenaris, D. Ho and T. J. Meade, Nano Lett., 2010, 10, 484.

175 S. J. McLachlan, M. R. Morris, M. A. Lucas, R. A. Fisco, M. N. Eakins, D. R. Fowler, R. B. Scheetz and A. Y. Olukotun, J. Magn. Reson. Imaging, 1994, 4, 301.

176 A. E. Stillman, N. Wilke and M. Jerosch-Herold, J. Magn. Reson. Imaging, 1997, 7, 765.

177 Y. Anzai, J. A. Brunberg and R. B. Lufkin, J. Magn. Reson. Imaging, 1997, 7, 774.

178 K. R. Hurley, Y.-S. Lin, J. Zhang, S. M. Egger and C. L. Haynes, Chem. Mater., 2013, 25, 1968.

179 P. J. Endres, T. Paunesku, S. Vogt, T. J. Meade and G. E. Woloschak, J. Am. Chem. Soc., 2007, 129, 15760.

180 W. J. Rieter, J. S. Kim, K. M. L. Taylor, H. An, W. Lin, T. Tarrant and W. Lin, Angew. Chem., Int. Ed., 2007, 46, 3680.

181 H. Yang, Y. Zhuang, H. Hu, X. Du, C. Zhang, X. Shi, H. Wu and S. Yang, Adv. Funct. Mater., 2010, 20, 1733.

182 F. Carniato, L. Tei, W. Dastru, L. Marchese and M. Botta, Chem. Commun., 2009, 1246.

183 F. Carniato, L. Tei, M. Cossi, L. Marchese and M. Botta, Chem. - Eur. J., 2010, 16, 10727.

184 J. L. Vivero-Escoto, K. M. L. Taylor-Pashow, R. C. Huxford, J. Della Rocca, C. Okoruwa, H. An, W. Lin and W. Lin, Small, 2011, 7, 3519.

185 D. Kryza, J. Taleb, M. Janier, L. Marmuse, I. Miladi, P. Bonazza, C. Louis, P. Perriat, S. Roux, O. Tillement and C. Billotey, Bioconjugate Chem., 2011, 22, 1145.

186 G. Liang, L. Cao, H. Chen, Z. Zhang, S. Zhang, S. Yu, X. Shen and J. Kong, J. Mater. Chem. B, 2013, 1, 629.

187 Y. Hou, R. Qiao, F. Fang, X. Wang, C. Dong, K. Liu, C. Liu, Z. Liu, H. Lei, F. Wang and M. Gao, ACS Nano, 2013, 7, 330.

188 Y. Sun, Q. Liu, J. Peng, W. Feng, Y. Zhang, P. Yang and F. Li, Biomaterials, 2013, 34, 2289.

189 Y. Liu, K. Ai, J. Liu, Q. Yuan, Y. He and L. Lu, Angew. Chem., Int. Ed., 2012, 51, 1437.

190 C. Haller and I. Hizoh, Invest. Radiol., 2004, 39, 149.

191 Y. Q. Wu, Y. Sun, X. J. Zhu, Q. Liu, T. Y. Cao, J. J. Peng, Y. Yang, W. Feng and F. Y. Li, Biomaterials, 2014, 35, 4699.

192 Z. Liu, F. Pu, S. Huang, Q. Yuan, J. Ren and X. Qu, Biomaterials, 2013, 34, 1712.

193 S. Zeng, M. K. Tsang, C. F. Chan, K. L. Wong and J. Hao, Biomaterials, 2012, 33, 9232.

194 C. Lutzweiler, R. Meier, E. Rummeny, V. Ntziachristos and D. Razansky, Opt. Lett., 2014, 39, 4061.

195 M. Gerling, Y. Zhao, S. Nania, K. J. Norberg, C. S. Verbeke, B. Englert, R. V. Kuiper, A. Bergstrom, M. Hassan, A. Neesse, J. M. Lohr and R. L. Heuchel, Theranostics, 2014, 4, 604.

196 S. K. Maji, S. Sreejith, J. Joseph, M. Lin, T. He, Y. Tong, H. Sun, S. W.-K. Yu and Y. Zhao, Adv. Mater., 2014, 26, 5633. 